\title{
The role of tradeoffs among life-history traits in a filamentous fungus
}
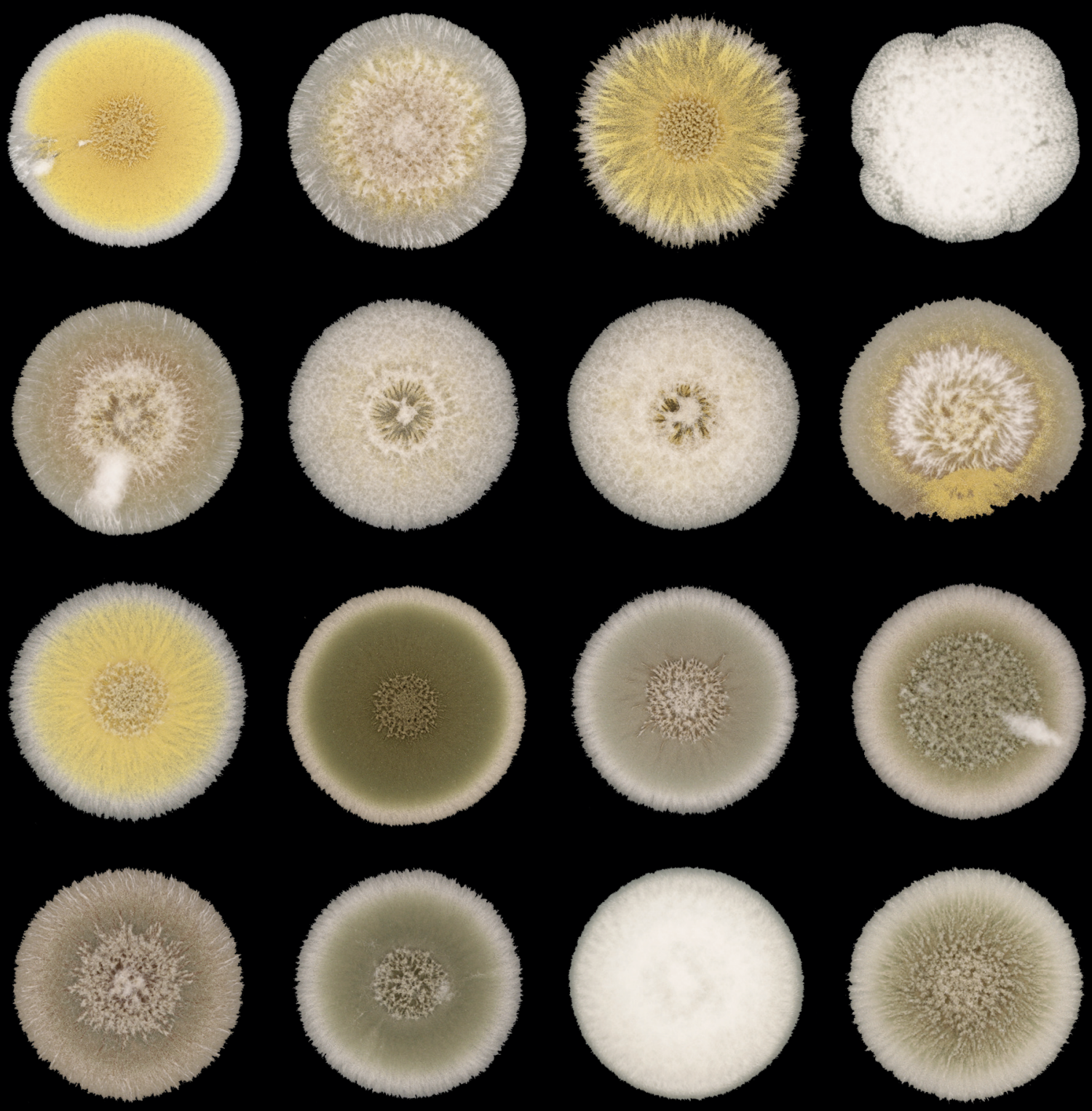

Krithi Nandimath 


\section{Propositions}

1. There is no trade-off between mycelial growth rate and spore production in Aspergillus nidulans.

(this thesis)

2. To fuse, or not to fuse, is the conundrum faced by evolving filamentous fungi.

(this thesis)

3. Civil engineers can learn from termites how to build climate-proof buildings.

4. Mandatory training in evolutionary biology for politicians will remedy future pandemics.

5. WUR needs to making printing theses optional to earn the label "most sustainable university".

6. University ranking criteria should include mental well-being of their faculty and students as a metric.

7. Doing a PhD does not mean that you give up your personal time and space.

8. The casual biking culture in The Netherlands is a good example of "niche construction" leading to "adaptive radiation".

Propositions belonging to the thesis, entitled

The role of tradeoffs among life-history traits in a filamentous fungus

Krithi Nandimath

Wageningen, 26 January 2022 


\section{The role of tradeoffs among \\ life-history traits in a filamentous fungus}

Krithi Nandimath 


\section{Thesis committee}

\section{Promotors}

Prof. Dr J. Arjan G. M. de Visser

Personal chair, Laboratory of Genetics

Wageningen University \& Research

Prof. Dr Duur K. Aanen

Personal chair, Laboratory of Genetics

Wageningen University \& Research

Prof. Dr B. J. Bas Zwaan

Professor of Genetics

Wageningen University \& Research

\section{Co-promotor}

Prof. Dr Vidyanand Nanjundiah

Centre for Human Genetics

Bangalore, India

\section{Other members}

Prof. Dr JE Kammenga, Wageningen University \& Research

Dr MGJ de Vos, University of Groningen

Prof. Dr HAB Wösten, Utrecht University

Dr AD van Diepeningen, Wageningen University \& Research

This research was conducted under the auspices of the C.T. de Wit Graduate School for Production Ecology and Resource Conservation 


\title{
The role of tradeoffs among \\ life-history traits in a filamentous fungus
}

\author{
Krithi Nandimath
}

\section{Thesis}

submitted in fulfilment of the requirements for the degree of doctor at Wageningen University

by authority of the Rector Magnificus

Prof. Dr A.P.J Mol, in the presence of the

Thesis Committee appointed by the Academic Board to be defended in public on Wednesday 26 January 2022 at 1:30 p.m. in the Aula. 
Krithi Nandimath

The role of tradeoffs among life-history traits in a filamentous fungus

140 pages

PhD thesis, Wageningen University, Wageningen, NL (2022)

With references, with a summary in English

ISBN

978-94-6447-066-6

DOI

https://doi.org/10.18174/560520 


\section{Contents}

$\begin{array}{lll}\text { Chapter } 1 & \text { General introduction } & 7\end{array}$

Chapter 2 Tests of a trade-off between growth and reproduction 23 among natural isolates of a filamentous fungus

Chapter 3 Adaptive dynamics of the fungus Aspergillus nidulans during 37 a one-year evolution experiment

Chapter 4 Test of metabolite interactions underlying experimentally 53 evolved polymorphisms in a filamentous fungus

Chapter 5 Genomic basis of a repeatedly evolved polymorphism in experimental populations of Aspergillus nidulans

Chapter 6 General discussion

References

Summary

Acknowledgements 


$$
1
$$




\section{CHAPTER 1.}

General introduction 
In this thesis, I explore the causes and constraints associated with adaptation in a simple multicellular organism, the fungus Aspergillus nidulans, with a special focus on the role of trade-offs between somatic growth and asexual reproduction. I start this chapter by introducing the model organism and modular organisms in general. Modular organisms are organisms, where each body part can reproduce, hence they have no germ-soma separation and are also theoretically immortal. Most fungi, including $A$. nidulans, fall into this category, while Podospora anserina is an exception. I explore the various definitions of an individual for modular organisms. Next, I describe life-history evolution and the role of trade-offs between fitness components, as well as the life-history strategies of filamentous fungi, and the role of the environment in trade-offs and adaptive divergence. I then introduce an important method used in this thesis, i.e., experimental evolution, and end the chapter with the outline of my thesis.

\section{The model organism Aspergillus nidulans}

The model organism used in this thesis is the ascomycete filamentous fungus Aspergillus nidulans. Aspergillus species grow on a wide range of carbon substrates and play an important role in degrading natural and artificial material (Baker \& Bennett, 2007). A. nidulans can undergo asexual and sexual reproduction and also has a parasexual cycle (figure 1.1). Germination of a uninucleate spore derived either asexually or sexually marks the beginning of the growth phase. The spore germinates and the resulting germ tube starts as a tubular hypha, that forms interconnected branches called a mycelium after a period of polarized growth. After 16 hours of growth, exposure of the mycelium to air and to red light or starvation of carbon and nitrogen triggers asexual reproduction, called conidiation. Differentiation begins at the center of the colony, where the mycelium branches to form aerial hyphae, and some of these form stalk-like structures: the conidiophores that bear the asexual spores or conidia. The conidiophores emerge on specialized thick-walled cells called foot cells (T. H. Adams et al., 1998).

The fusion of two vegetatively growing hyphae to form a heterokaryon or a dikaryotic hypha marks the beginning of the sexual cycle. A. nidulans is homothallic, that is, it can self-fertilize. The initial dikaryotic cell formed after fertilization expands to form a network of ascogenous dikaryotic hyphae within the differentiating fruiting body called cleistothecium. These ascogenous hyphae undergo nuclear fusion and each diploid undergoes meiosis and a post-meiotic mitosis forming eight haploid ascospores included in an ascus. A mature cleistothecium may eventually contain more than hundred thousand ascospores derived from over ten thousand meiosis. 
The parasexual cycle involves recombination in the absence of sexual reproduction. A heterokaryon (i.e., mycelium containing two haploid nuclear genotypes) produces a diploid nucleus by nuclear fusion, which undergoes spontaneous mitotic recombination with an independent assortment of chromosomes during haploidization (Pontecorvo, 1956; Pontecorvo et al., 1953).

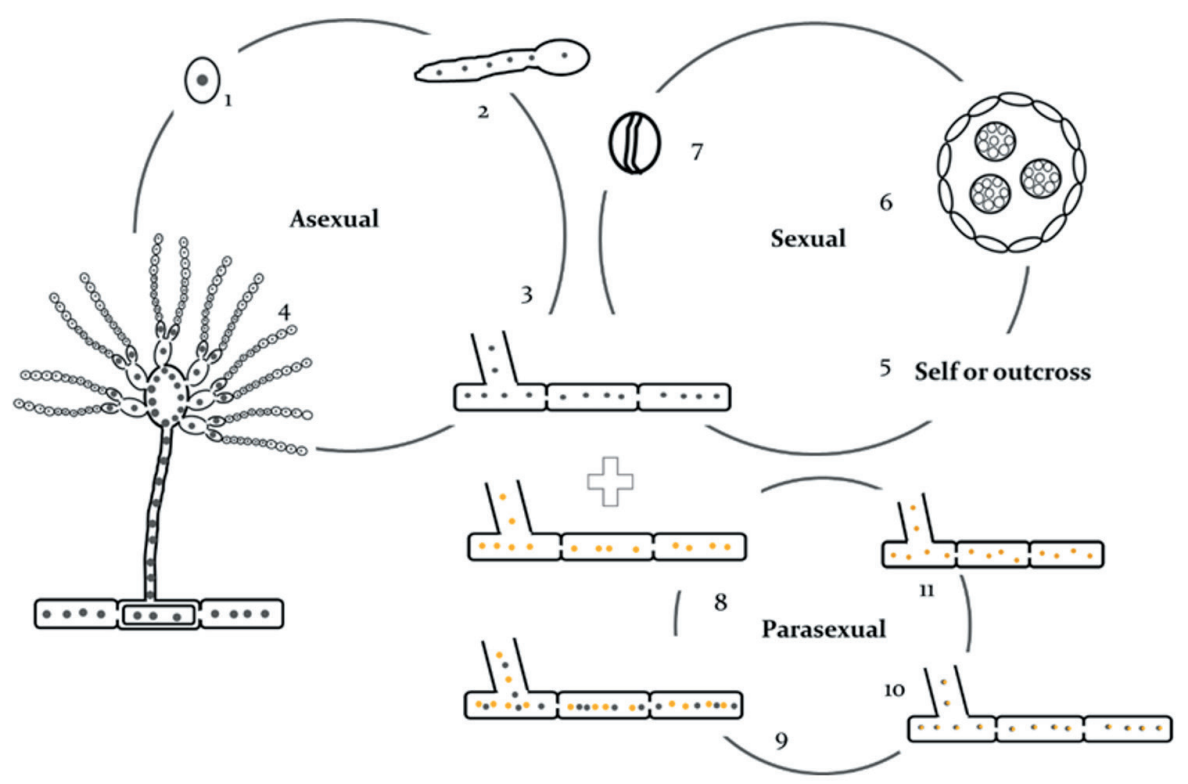

Figure 1.1: Life cycle of Aspergillus nidulans. The asexual life cycle (steps 1-4) begins with the germination of a conidium that grows to form an interconnected mycelial network, which upon environmental and genetic cues forms conidiophores that bear many asexual spores, called conidia. The sexual life cycle (steps 5-7) starts with fusion of two mycelia and results in the formation of a fruiting body, called cleistothecium. Each ascus inside a cleistothecium contains eight haploid ascospores, resulting from nuclear fusion, followed by meiosis. In the parasexual cycle (steps 8-11) two haploid mycelia fuse to form a heterokaryon that continues to divide mitotically.

\section{Unitary and modular organisms}

We can group organisms into unitary and modular organisms, based on their body plan. Unitary organisms have a determinate body plan with specialized organs and early germline-soma separation, and are subjected to aging and eventually death. Somatic cells are supportive cells that form the body and all its tissues whereas the germline cells produce the reproductive cells and thus cause the perpetuation of life from generation to generation. In contrast, organisms with modular body plans have no germline-soma separation and are theoretically immortal (figure 1.2). Such modular organisms are composed of modules that are self-sustaining, repetitive, and totipotent. The modules either remain attached or separate from other modules to start a new colony. Examples of modular organisms are plants, sessile animals such as hydroids and bryozoans, and fungi (Vuorisalo \& Tuomi, 1986). 


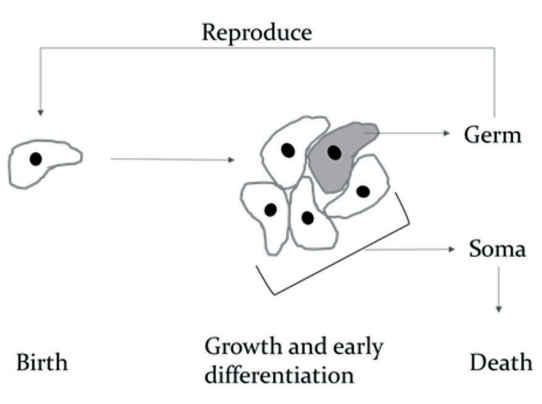

Unitary organisms
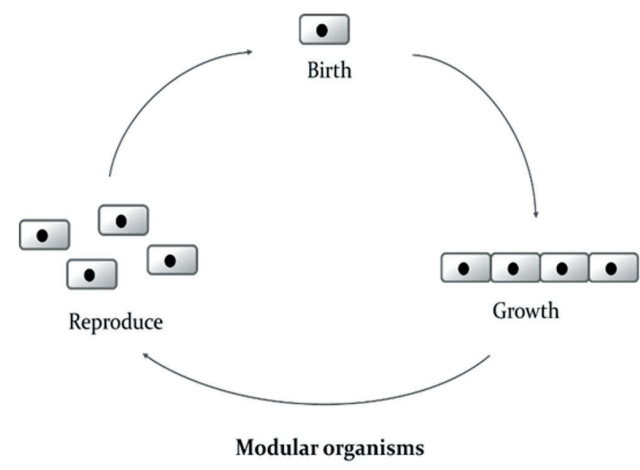

Figure 1.2: Schematic of differences between unitary and modular organisms. Unitary organisms (left) grow, undergo early cellular differentiation into somatic cells and germ cells. Only the germ cells retain the capacity to reproduce and the somatic cells help in maintenance of the organism and will eventually die. In modular organisms (right), all cells are capable of reproduction and theoretically immortal.

\section{Defining an individual}

Darwin and Wallace's theory of evolution by natural selection provides a scientific explanation for how living organisms have adapted to the environments that they inhabit (Ayala, 2007; Charlesworth \& Charlesworth, 2003). Adaptative evolution is a process by which organisms acquire, modify or fine-tune a trait or character to enable their survival and reproduction in a given environment, and natural selection is a mechanism that enables adaptive evolution (Lewontin, 1978). Darwin and Wallace saw the population as the smallest unit capable of evolving. Individuals in the population show heritable variation in reproduction and survival, interact with the environment and are the units on which natural selection acts. This idea is applicable to unitary organisms, where an individual is the end-point of development. Because of early sequestration of the germ cells in unitary organism selection does not act on intra-individual variation occurring due to somatic mutations.

How is an individual defined in modular organisms? The defining features of a modular organism are developmental and phenotypic iteration, somatic embryogenesis, and semi-autonomy in maintenance and reproduction. Therefore, a module is the reproductive unit of modular organisms (Vuorisalo \& Tuomi, 1986). Santelices (1999) summarized the classical attributes of an individual as: (i) genetic uniqueness, (ii) genetic homogeneity, and (iii) autonomy and physiological unity, which are most suited for unitary organisms (Santelices, 1999). All modular organisms refute the first classical attribute of genetic uniqueness since they reproduce asexually, and genetically identical units can independently survive and replicate. The mycelium of a filamentous fungus is not always genetically 
homogenous as it can fuse with other compatible mycelia. This provides modular organisms with unique abilities, such as utilizing novel sources of nutrients, which each genetically homogeneous mycelium could not on its own.

Folse III and Roughgarden (2010) define an individual based on the evolutionary concept of fitness. According to them, (i) an individual organism is one where the fitness interests of all its components are aligned. Genetic relatedness of the components is essential for aligning fitness interests and introducing bottlenecks (Grosberg \& Strathmann, 1998, 2007) and allorecognition (Czárán et al., 2014) in the life-cycle of the individual can ensure genetic relatedness. (ii) An individual organism is one where its components are interdependent on one another for reproduction. In such situations, fitness is exported from a lower level to a higher level by division of labor, for example, by germ-soma specialization (Michod, 2005). The alignment of fitness suggests that cooperation must evolve, whereas, for the export of fitness to a higher level, there must be interactions among the components (Folse III \& Roughgarden, 2010).

The transfer of fitness from a lower to a higher level also occurs during evolutionary transitions of individuality (ETI). Maynard Smith and Szathmáry (1995) defined ETI as those transitions in which units of selection changed from lower levels (independent entities) to higher levels (groups of previously independent entities). The entities capable of autonomous replication can now only replicate as part of a whole after the transition (figure 1.3). Some major evolutionary transitions include the formation of cells with compartmentalized replicating molecules, the emergence of eukaryotic cells with endosymbionts, the evolution of multicellularity from unicellular ancestors, and the formation of societies with formerly independent multicellular individuals (Maynard Smith \& Szathmary, 1995).

During the early transition to multicellularity, when cells began aggregating in groups, the group's fitness was the average fitness of the individual cells of the group. This stage in multi-level selection is known as multi-level selection I (MLS I). As complexity evolved, the fitness of the group was no longer just the average fitness of its constituents, but the survival and reproduction of its progeny. This later stage in multi-level selection is termed multi-level selection II (MLSII) (Damuth \& Heisler, 1988). The difference between MLS I and MLS II helps to understand the transfer of fitness from cells to cell groups during evolutionary transitions of individuality (Okasha, 2005). 
A filamentous fungus shares some features of the 'alignment of fitness' definition of an individual. For instance, it has mechanisms like allorecognition to prevent fusion with unrelated individuals. The fungus shares features with the 'export of fitness' definition of an individual; the mycelium can be equated to the soma and the spores as the germline; however, the soma in the fungus has not given up its reproductive rights, like in unitary organisms. Therefore, if variation is expressed at various levels of biological organization, then selection may act at multiple types of replicating units simultaneously and not just at the level of the unitary individual (Fagerström et al., 1998; Tuomi \& Vuorisalo, 1989a). For example, the somatic mutations expressed in the clonal seagrass Zostera marina can be incorporated into propagative cells causing intra-clonal variation, which might alter the reproductive output of modules of a structural individual (L. Yu et al., 2020).

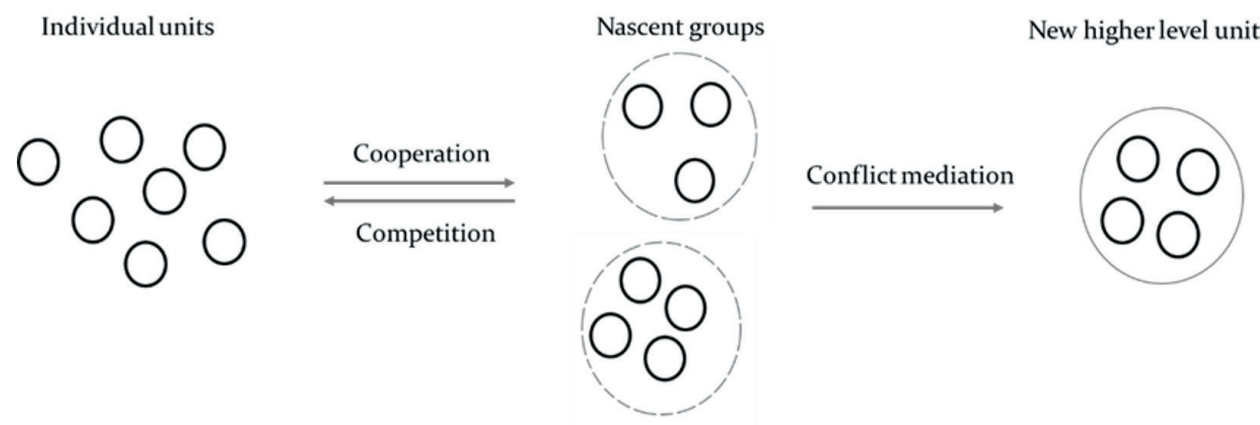

Figure 1.3: Steps during the evolutionary transitions in individuality. Cooperation among individual units result in the formation of nascent groups and competition between individuals within a nascent group causes the breakdown of the group. A new higher level unit forms when there is a mechanism for conflict mediation and differential fitness between these higher-level units transfers the fitness from individual units to groups of individuals.

\section{Life-history evolution}

Life-history theory explains how natural selection optimizes an organism's investments in fitness components, such as growth and reproduction, to achieve reproductive success. Adaptation by natural selection depends on heritable variation in fitness components. Since life-history traits determine an organism's fitness, understanding life-history strategies implicates understanding adaptation in the context of the biology of the organism in its natural habitat (Fabian \& Flatt, 2012). Life-history theory explains the variation seen in growth rates, size at birth, age and size at maturity, reproductive investments, mortality rates, and lifespan (Stearns, 2000). As contrasting examples, bamboo plants and Chinook salmons reproduce once and then die, termed semelparity, whereas Atlantic salmons and oak trees reproduce several times in their lifetime, termed iteroparity. To understand lifehistory evolution, one has to understand how environmental factors impact survival 
and reproduction, as well as the correlation between fitness-related traits, including the role of trade-offs among life-history traits (Stearns, 2000).

A trade-off is defined as a negative correlation between fitness components, across individuals or genotypes, and may pose constraints on life-history evolution and adaptation (Acerenza, 2016). At the physiological level, trade-offs may be caused by the competitive allocation of limited resources to one life-history trait instead of the other within a single individual, as van Noordwijk and de Jong (1986) described in the Y-shaped resource-allocation and acquisition model. For example, investment in reproduction may be favored in abundant-nutrient conditions, and energy from reproduction diverted into somatic maintenance and survival under nutrient limitation (Boggs, 2009; Burger et al., 2019; Zera \& Harshman, 2001). Studying trade-offs in cellular processes like the rate and yield of ATP production (Pfeiffer et al., 2001) and growth rate and yield in bacterial populations (Novak et al., 2006) may help our understanding of the mechanistic basis of trade-offs arising due to limitations from resource acquisition and allocation.

An alternate cause of trade-offs between survival and reproduction, unrelated to energy budgeting, is that reproduction either damages or inhibits somatic maintenance and survival (Flatt, 2011). The key findings from studies on the cost to reproduction reviewed in (Flatt, 2011) are that trade-offs between survival and reproduction are widespread. These life-history traits can be uncoupled under certain conditions (for example, by mutations in insulin-like growth factor receptors), as observed in Caenorhabditis elegans and Drosophila melanogaster (Flatt, 2011).

\section{Life-history strategies for a filamentous fungus}

The evolution of life-history traits, like size at birth, size at maturity, size and number of offspring, age at reproduction, and longevity, are affected by tradeoffs (Stearns, 1989). The best-studied among these is the trade-off between reproduction and longevity in unitary organisms (Bleu et al., 2016; Charnov \& Berrigan, 1991; Flatt, 2011; Harshman \& Zera, 2007; Reznick, 1983). What is an optimal life-history strategy for a modular filamentous fungus?

When all traits of two fungal genotypes are equal except their growth rate, then the species with a higher growth rate will outcompete the one with a lower growth rate, as the one with a higher growth rate captures a larger fraction of the available resources. Similarly, when the only difference between two genotypes is their reproductive output, the species producing more offspring will outcompete the one with fewer offspring. However, these two traits, growth and reproduction, 
are likely to trade off as the energy spent on one trait may not be available for the other (Heaton et al., 2016).

Gilchrist et al., (2006) have developed a patch-array model focusing on the lifehistory strategies that a fungus can employ when growing on limited resources. They ask a simple life-history question, i.e., how does the decision to allocate resources towards growth or reproduction change for a fungus growing on a limited patch of resources? Investing in spore production leads to a direct fitness benefit; however, investing in mycelium provides possible indirect, i.e., future, benefits via the greater extraction of resources which may enhance future reproduction. Their model demonstrates that under finite resource conditions, the fungus employs a so-called "bang-bang life-history strategy". In a bang-bang lifehistory, the resources are first allocated towards growth, and at a critical point, the resources are allocated towards reproduction. The optimal timing for switching to reproduction, in a bang-bang strategy, will vary depending on resource removal rates, probability of disturbance to the resource patch before reproduction, and competition with other species/genotypes (Gilchrist et al., 2006; Heaton et al., 2016). A fungus growing on a large, durable resource, such as dead trees, will likely increase the chance of their offspring survival if they grow as rapidly as possible, and reproduce late and allocate only a small fraction of the available resources to reproduction (Table 1.1) (Heaton et al., 2016).

Fungal mycelium exploits nutrient-rich environments by producing dense, compact mycelium (high biomass) with increased hyphal branching for resource exploitation, whereas fungi growing on nutrient-poor medium produce mycelia with reduced branching (less dense, low biomass) for nutrient exploration (Ritz, 1995; Whiteway \& Bachewich, 2005). Suppose the total number of spores produced by a colony in a given environment are constant; in that case, the total spores produced by both the exploration and exploitation strategy remain the same. Still, the spore density per surface area is reduced in the exploitation strategy. Schoustra and Punzalan (2012) found a negative correlation between radial colony growth and total spore 


\begin{tabular}{|c|c|}
\hline Condition & Response \\
\hline \multicolumn{2}{|l|}{ A: Resource quality } \\
\hline Rich resource & Mycelium dense and compact for resource exploitation \\
\hline Poor resource & Mycelium thin for resource exploration \\
\hline \multicolumn{2}{|l|}{ B: Resource durability } \\
\hline $\begin{array}{l}\text { Short-lived resources } \\
\text { (e.g., dung or rotting fruit) }\end{array}$ & $\begin{array}{l}\text { Fast mycelial growth for resource capture and then one-time } \\
\text { reproduction after resource capture ("bang-bang life-history") - } \\
\text { semelparous life style }\end{array}$ \\
\hline $\begin{array}{l}\text { Long-lived resources } \\
\text { (e.g., dead tree) }\end{array}$ & $\begin{array}{l}\text { Slower growth and late reproduction start, with multiple repro- } \\
\text { ductive periods - iteroparous life style }\end{array}$ \\
\hline \multicolumn{2}{|l|}{ C: Presence of competition } \\
\hline $\begin{array}{l}\text { No interaction between } \\
\text { individuals }\end{array}$ & Grow fast to monopolize resources \\
\hline $\begin{array}{l}\text { Metabolic interactions } \\
\text { between individuals }\end{array}$ & Engage in cross-feeding or interference competition \\
\hline \multicolumn{2}{|c|}{ D: Conditions causing positive, negative or no MGR-spore yield correlation } \\
\hline $\begin{array}{l}\text { Novel condition causing low } \\
\text { fitness }\end{array}$ & $\begin{array}{l}\text { No tradeoff, growth rate and reproduction may be positively } \\
\text { correlated and both may improve adaptively }\end{array}$ \\
\hline $\begin{array}{l}\text { Condition to which the fungus } \\
\text { is relatively well adapted }\end{array}$ & $\begin{array}{l}\text { Tradeoff between growth rate and reproduction may be apparent } \\
\text { and constrain further adaptation }\end{array}$ \\
\hline
\end{tabular}

Table 1.1: Overview of the various life-history options that a fungus may adopt based on environmental conditions.

(A) Resource quality, (B) Resource durability, (C) Presence of multiple individuals. (D) Describes general conditions for a tradeoff between mycelial growth rate and asexual spore yield. The alternate strategies mentioned in (A-C) are not necessarily mutually exclusive and most fungi are likely to employ a combination of these strategies based on ecology and organismal biology.

production per surface area in Aspergillus nidulans, supporting a trade-off between mycelial growth rate and spore density and also total spore yield.

\section{Ecological opportunities for adaptive trade-offs and divergence}

Environmental heterogeneity at both macro and microscopic spatial scales may cause individuals of the same species to experience different selection pressures based on the environment they are adapting to, leading to local adaptation. For example, when Becker et al., (2006) reciprocally transplanted the seedlings of Carlina vulgaris, a grassland perennial from different altitudes, they found evidence for local adaptation (Becker et al., 2006). Local adaptation may produce specialists customized to their specific environment with lower relative fitness in alternate environments, seen from trade-offs in performance across environments (Bono et al., 2017; Gorter et al., 2015; Hereford, 2009).

Extensive local adaptation is seen during adaptive radiation, a process where descendants from a common ancestor diversify by adapting to diverse niches (Gillespie et al., 2001; MacLean, 2005). Understanding how mutations, the raw 
material for selection, translate to variation in adaptive phenotypes is essential for understanding the constraints experienced during adaptation. A classical study by Rainey and Travisano (1998) observed three morphotypes of the bacterium Pseudomonas fluorescens to repeatedly evolve in a structured environment via adaptive radiation to different regions within the culture. The structured environment created specific niches with varying oxygen concentrations to which the different morphs repeatedly adapted and stably coexisted by negative frequency-dependent fitness interactions (the fitness of a genotype decreases as its frequency increases since the fitness of one type is dependent on the other) (Lind et al., 2015; Rainey \& Travisano, 1998).

Within the Escherichia coli long-term evolution experiment (LTEE), which uses a simple homogeneous environment (Lenski et al., 1991), several populations evolved semi-stable polymorphisms, involving adaptation to different niches. Population Ara-3, where a citrate utilizing $\left(\mathrm{Cit}^{+}\right)$genotype evolved with the ancestral citrate non-utilizing (Cit) genotype by acquiring the ability to use citrate (which was also present in the evolution medium) as a carbon source in addition to glucose (Blount et al., 2008). The evolution of cross-feeding between the Cit+ and ancestral Citgenotypes ensured their stable maintenance: the Cit genotype evolved a metabolic dependence on the Cit+ type by cross-feeding on c4 dicarboxylate metabolites produced by the $\mathrm{Cit}^{+}$genotype. The cross-feeding interaction converted a dual carbon environment (i.e. glucose and citrate) into one with multiple carbon sources shared or portioned between the types (Blount et al., 2012; C. B. Turner et al., 2015). Other examples of evolved cross-feeding interactions, where one genotype feeds on an available carbon source, and the other genotype uses the metabolite excreted by the first as a source of energy include (Helling et al., 1987; Rosenzweig et al., 1994; Treves et al., 1998; P. E. Turner et al., 1996). In other LTEE populations, complex mechanisms involving trade-offs between resource competition and death rates in the stationary phase, combined with cannibalism, helped maintain diversity (Rozen et al., 2009; Rozen \& Lenski, 2000). Multiple niche availability is an essential requirement for maintaining diversity over long time scales by negative frequency-dependent fitness interactions (France \& Forney, 2019; Habets et al., 2006; Jousset et al., 2016).

Multiple niches can be created by varying abiotic components, like the duration of exposure to light and using combinations of limiting nutrients (Bell \& Reboud, 1997; T. F. Cooper \& Lenski, 2010; Saxer et al., 2010). Biotic components of the environment can also create multiple niches, for example, when different predators challenge a prey population or vice versa. Friman and Buckling (2013) studied P. fluorescens (prey) diversification when challenged with different types of phages. Others 
have studied the diversification of predators as the prey diversified. Paterson et al., (2010) studied the evolution of phage $\Phi$ (predator) under coevolving and naïve conditions of P. fluorescens (prey). Meyer et al., (2016) studied the evolution of a generalist phage lambda as it evolved into diverse specialists when cultured in the presence of diverse hosts expressing different receptors for phage lambda infection. However, not all heterogeneous environments lead to the evolution of diversity (T. F. Cooper \& Lenski, 2010; Saxer et al., 2009). The lack of diversification can be either due to limited evolutionary time or available genetic variation, or the lack of trade-offs between specialist traits (Van den Bergh et al., 2018).

Negative frequency-dependent fitness interactions can also be seen with multiple interacting species, for example, in biofilm communities (V. S. Cooper et al., 2014; Flynn et al., 2016; Hansen et al., 2007; Traverse et al., 2013), or among coexisting mutualist partners (D'Souza \& Kost, 2016). In predator-prey systems, frequency dependence combined with arms race dynamics (where competing species/ genotypes adapt to each other) helps maintain diversity and stable coexistence (Hall et al., 2011).

When evolving genotypes change the environmental conditions, such as in the examples above, fitness interactions may become non-transitive. Non-transitive fitness interactions occur when the fitness of evolved genotypes relative to their ancestor cannot be predicted from the incremental improvements relative to more immediate predecessors (De Visser \& Lenski, 2002; Kerr et al., 2002; Van den Bergh et al., 2018). Such fitness interactions are likely to evolve when the environment is heterogeneous and performance trade-offs between niche specialists arise, or when evolving genotypes change the selective conditions, e.g., through cross-feeding or the costly production of anti-competitor toxins (De Visser \& Lenski, 2002). Three $E$. coli genotypes: toxin producer, a toxin resistant mutant, and a toxin-sensitive strain were maintained in a population by non-transitive fitness interactions. Diversity in these populations could be maintained when the interactions were localized and lost when interactions occurred over large spatial scales (Kerr et al., 2002). Some evolution experiments have reported non-transitivity of fitness interactions (Beaumont et al., 2009; Buskirk et al., 2020; Hall et al., 2011; Kerr et al., 2002; Paquin \& Adams, 1983). Buskirk et al., (2020) demonstrated that in populations of Saccharomyces cerevisiae, non-transitive fitness interactions could occur as a result of multi-level selection on the host genome and an intracellular viral genome producing metabolically costly anti-competitor toxins and immunity factors. The adaptive mutations resulted in the loss of toxin production and loss of immunity against the toxin in a sequential manner. Thus, when the evolved populations were competed with the ancestor (with a functional toxin producing viral genome), the evolved genotypes were killed 
by the toxin- producing ancestors resulting in non-transitive fitness interactions (Buskirk et al., 2020). Non-transitive fitness interactions may also be responsible for the common observation of a deceleration of fitness improvements relative to the ancestor in laboratory evolution experiments, but this was not the case for one population of LTEE tested after 20,000 generations (De Visser \& Lenski, 2002).

\section{Microbial experimental evolution}

Microbial experimental evolution uses mostly bacteria, viruses, algae, amoebae, unicellular and multicellular fungi to test evolutionary theories and help find ecological causes and causal genotypes of certain adaptive phenotypes. Microbes are excellent candidates for experimental evolution, because they have short generation times, and one can culture large populations in small spaces. Moreover, they can be stored as "frozen fossils" and revived later for direct comparison, and microbes reproduce asexually, which helps maintain clonality and linkage between the genetic marker and the genomic background. The design of an evolution experiment is often simple: replicate populations are established from a single ancestral clone and propagated for many generations in a controlled, reproducible environment. At regular intervals, samples of the evolving populations are stored. After evolution, changes in the phenotypic and genetic properties of interest are measured and compared with the ancestor and the evolved populations and among evolved populations. Such comparisons help understand the dynamics and repeatability of evolutionary change and its genetic and ecological causes and constraints (Elena \& Lenski, 2003; Van den Bergh et al., 2018).

In the asexual, initially isogenic, populations used in experimental evolution, random mutations are the only source of variation for natural selection to act on. Absolute fitness and relative fitness are two ways of quantifying the progress of adaptation. Absolute fitness is expressed as differences between the ancestor and evolved populations in fitness components such as growth rate (Fong \& Palsson, 2004; LaCroix et al., 2015), yield (Avrahami-Moyal et al., 2012; Friman et al., 2015; Ketola \& Saarinen, 2015) and lag phase (Bertranda, 2019; Vasi et al., 1994). Relative fitness is usually measured as fitness of an evolved population or clone relative to the ancestor in direct head-to-head competition in the evolutionary environment. Relative fitness is often calculated as the ratio of the growth rates of the ancestor and evolved genotype, inferred from their initial and final frequencies (Lenski et al., 1991). A common theme that has emerged across studies with viruses and bacteria is that fitness improvements are initially rapid and decrease over time (Lenski et al., 1991; Lenski \& Travisano, 1994; Novella et al., 1995). However, the potential for genetic adaptation is not exhausted even in a constant environment after tens of thousands of generations (Wiser et al., 2013). 
Multicellular fungi are also excellent model systems for experimental evolution. They combine the complexity of multicellular eukaryotes with short generation times, storage as frozen fossils, and similar ease of manipulation as offered by single-celled organisms. However, their life cycle includes the development of somatic and reproductive tissues, which raises the question of how these different stages contribute to adaptation in a novel environment (Fisher \& Lang, 2016).

Filamentous fungi have been used in experimental evolution to address a range of topics; for example, the role of environmental structure (Kudryavtseva et al., 2019), epistasis (Schoustra et al., 2016), bet-hedging (Graham et al., 2014), and rate and distribution of mutations (Gifford et al., 2011; Schoustra et al., 2009) during adaptation. Fungi have been used to address the evolution of antimicrobial resistance and the role of compensatory mutations during evolution of antimicrobial resistance (Schoustra et al., 2006; Zhang et al., 2015). Filamentous fungi have also been used to understand the effects of (i) relatedness in maintaining multicellular cooperation, coevolution, and competition (Bastiaans et al., 2016; Grum-Grzhimaylo et al., 2021; Trienens \& Rohlfs, 2011), (ii) reproductive isolation, speciation and sexual selection (Dettman et al., 2008; Nieuwenhuis \& Aanen, 2018), (iii) effects of ploidy, nuclear co-adaptation and mitotic recombination (Clark \& Anderson, 2004; Schoustra et al., 2007).

\section{Setup of the thesis}

This thesis describes the results of experiments with the filamentous fungus $A$. nidulans. It addresses the mode of adaptation of this relatively simple and modular multicellular organism to a new environment using experimental evolution as a tool. Chapters $\mathbf{2}$ and $\mathbf{3}$ describe the results of different evolution experiments with $A$. nidulans. There are both similarities and differences in the design of these experiments. For instance, both experiments make use of a structured synthetic minimal medium as the evolution environment. The differences in their designs are as follows. In chapter $\mathbf{2}$, the standard carbon concentration of $1 \%$ glucose is used and the colony grows radially from a central point as a single individual on the surface of a $90 \mathrm{~mm}$ Petri plate for four days. When populations are growing radially from a central point, selection can favor mutations that increase the colony area, thereby increasing the area available for resource exploitation and sporulation. This would mean that fitness would increase even with reduced per area sporulation as long as the total sporulation is higher than that of the other. Alternately, it can also favor mutations that increase the biomass (dense mycelium) to help capture resource efficiently and improve the density of spores. Whereas, in chapter $3,10 \mathrm{X}$ reduced carbon for growth ( $0.1 \%$ glucose) is used, and a high density of spores is spread on the surface of a bottle with an approximate diameter of $3 \mathrm{~cm}$ leading to 
multiple colonies growing adjacent to each other. The fungus completely covers the agar's surface within 48 hours and is only transferred after seven days. These environmental factors might play a role in resource allocation decisions during adaptation. For instance, competition for space and resources between multiple colonies might play a role in the ASEX populations (chapter 3 ) since it is evolving on limited carbon and space.

Chapter $\mathbf{2}$ explores trade-offs between growth and reproduction at two timescales. Firstly, trade-offs resulting from local adaptation are examined by measuring the mycelium growth rate and asexual spore yield of a set of natural isolates of $A$. nidulans by altering the nutritional complexity and energy source availability. Secondly, by exploiting the quick doubling time and simple life-cycle of A. nidulans, a short evolution experiment is set up to test for possible short-term adaptive constraints from trade-offs between growth and reproduction under controlled environmental conditions among evolved populations. We did not find evidence for long-term adaptive constraints from a trade-off between growth and asexual spore yield among the natural isolates tested. However, we found growth and asexual sporulation to respond in an antagonistic manner, suggesting shortterm adaptive constraints from a trade-off, during adaption in the laboratory.

Chapters 3 and $\mathbf{4}$ explore the phenotypic basis of adaptation of a laboratory strain of $A$. nidulans during a year of adaptation to growth and reproduction on solid media with limiting nutrients. The adaptive dynamics and strategies of the fungus have been addressed by analyzing changes in the competitive ability of the evolved populations relative to the ancestors. Most of the populations rapidly evolved a polymorphism, involving at least two morphotypes, and the transitivity and frequency-dependence of fitness interactions were analyzed to understand their adaptive role. Possible metabolic interactions among clones representing different morphotypes from the same evolving populations, which may underlie these fitness differences were investigated. We found evidence for nontransitive and frequency-dependent fitness interactions between two dominant morphotypes. The co-existence of the morphotypes can be partially explained as a result of resource competition between them.

Chapter 5 explores the genomic bases for the phenotypic adaptation of the ASEX populations. The role of natural selection has been addressed by measuring relative rates of non-synonymous and synonymous mutations and parallel genetic changes and examining possible functions of the genes under selection in our experimental setup. The genomics data showed a clear role of natural selection in the evolving ASEX populations, where genes involved in secondary metabolism and fusion are targets of selection. 


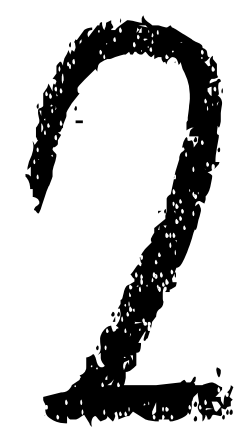




\section{CHAPTER 2.}

\section{Tests of a trade-off between growth and reproduction among natural isolates of a filamentous fungus}

Krithi Nandimath 1,2, Julia Marschall 1', Sijmen E. Schoustra ${ }^{1}$, Alfons J. M. Debets ${ }^{1}$, Vidyanand Nanjundiah ${ }^{2}$, Duur K. Aanen ${ }^{1}$ and J. Arjan G. M. de Visser ${ }^{1}$

${ }^{1}$ Laboratory of Genetics, Wageningen University, Wageningen, The Netherlands.

${ }^{2}$ Centre for Human Genetics, Bangalore, India. 


\section{Abstract}

The life history of an organism reflects the balance in investments in fitnessrelated traits and poses constraints for future evolution. Such constraints can be weak and quickly broken by adaptation or strong with long-term consequences for the rate and direction of adaptation. In this study, we tested for trade-offs between two life-history traits, growth and asexual reproduction, in natural isolates of the filamentous fungus Aspergillus nidulans. We first tested for tradeoffs due to local adaptation by growing the isolates in four different laboratory environments with varying nutrient richness and carbon source availability. We did not find evidence of a trade-off between growth and asexual reproduction in any of the four environments. However, we did see a positive correlation when comparing carbon source availability and nutrient richness conditions, which likely reflects deep environment-dependent developmental programs. We then used a 10-week evolution experiment with a subset of natural isolates to test for shortterm evolutionary constraints on a single trait (opposing selection on a single trait) or on multiple traits due to antagonistic pleiotropy. The data from the evolution experiment showed a negative correlation between growth and spore density, but not between growth and total spore yield, indicating that adaptation to the laboratory resulted in improvements in hyphal expansion without effect on total asexual spore production. Our findings suggest that trade-offs between growth and reproduction do not pose strong adaptive constraints in this filamentous fungus. 


\section{Introduction}

How organisms balance investments in growth and reproduction determines their "life history" and reflects the constraints encountered during their evolutionary history (Schluter et al., 1991). Life history theory aims to explain how ecological forces have driven organisms to optimize their survival, growth, and reproduction and consequently much of the diversity of the natural world. It also helps to understand adaptation by natural selection, which acts on variation in Darwinian fitness components among individuals. If life-history traits were uncorrelated, then selection would maximize their contribution to fitness independently. However, in reality, the fitness contributions of different life-history traits are often constrained, leading to trade-offs between traits (Stearns, 1989).

Trade-offs are described as a negative correlation between fitness components of individuals in a population. At the genetic level, trade-offs are caused by antagonistic pleiotropy or mutation accumulation. Antagonistic pleiotropy occurs when mutations that positively affect one fitness component affect another fitness component negatively. For example, in Drosophila melanogaster, selection for increased lifespan was coupled to reduced early reproduction through antagonistically pleiotropic alleles (Zwaan et al., 1995). Mutation accumulation occurs when unused functions decay by genetic drift in the absence of selection. At the physiological level, trade-offs may arise when resources are limited and insufficient to maintain two traits that share the resource. Increasing the resource allocated to one trait will result in a decrease of resources for the other. In their Y-shaped model, de Jong \& van Noordwijk (1986) have shown how resources not allocated to growth will be invested in reproduction when resources are limited and that there is individual variation in the acquisition of absolute resources. Based on the differences in acquisition and allocation, their model suggests when survival and reproduction will covary positively or negatively. Studying trade-offs in cellular processes, such as the rate and yield of ATP production in organisms (Pfeiffer et al., 2001) and growth rate and yield in bacteria (Novak et al., 2006), may help our mechanistic understanding of trade-offs arising due to limitations from resource acquisition and allocation.

Populations growing in different environments experience diverse selection pressures and are likely to follow distinct evolutionary trajectories resulting in genotype-by-environment ( $G X E$ ) interactions for Darwinian fitness. In the absence of drift and gene flow, such $G X E$ interaction should lead to the selection of genotypes with better-adapted traits to their local environment irrespectively of the effects of those traits in alternate environments (Acerenza, 
2016; Kawecki \& Ebert, 2004). Local adaptation is measured as fitness trade-offs across environments and is frequently observed in natural populations (Hereford, 2009). The populations of the long-term evolution experiment with Escherichia coli (LTEE) (Lenski et al., 1991) lost their ability to use 16 of the 64 different carbon sources tested after 10,000 generations. Since this loss was accompanied by a fitness increase in the presence of glucose, which was the sole carbon source in the evolutionary medium, particularly early during the experiment, the authors concluded that antagonistic pleiotropy most likely caused these trade-offs (Cooper \& Lenski, 2000).

In this study, we tested for adaptive constraints among two fundamental lifehistory traits of the filamentous fungus Aspergillus nidulans, mycelium growth rate, and asexual reproduction. The life cycle of $A$. nidulans starts with the germination of a single spore, producing a network of multinucleate hyphae. After a period of growth, triggered by environmental and genetic cues, the mycelium develops foot cells that produce aerial conidiophores bearing uninucleate asexual spores the conidia, that subsequently spread by air. Apart from asexual reproduction, $A$. nidulans may also reproduce sexually and parasexually (Pontecorvo, 1953).

A. nidulans is commonly found in soil and on rotting fruits, and strains can utilize a variety of carbon substrates for growth in the laboratory, from simple sugars to complex carbohydrates (Baker \& Bennett, 2007). Asexual conidiation can be induced by environmental factors like the exposure of the colony to air interface and light, but not before a period of vegetative growth (Axelrod, 1972; Noble \& Andrianopoulos, 2013). The timing of acquiring reproductive competence is directly correlated to organismal density and is presumably a response to resource colonization (Noble et al., 2016). The timing of sporulation is predicted to reflect life-history optimization to growth on patchy resources (Heaton et al., 2016). Small patches and intense competition is predicted to accelerate the timing of sporulation initiation and vice-versa (Gilchrist et al., 2006; Heaton et al., 2016). We grew natural isolates from various niches in four different nutrient environments to test the constraints from diverse evolutionary histories on growth rate and spore yield. Here, an observed trade-off would suggest evolutionary constraints due to adaptive specialization to their local environment.

Gifford et al. (2013) modeled fungal colony population growth and found that changes in mycelial growth rate had the most significant positive effect on the production of spores in a rich solid environment. In the absence of genetic constraints or covariances between these traits (MGR and sporulation), both of these traits can be candidates for adaptation (Gifford \& Schoustra, 2013). 
However, rapid growth and sporulation may be in conflict because resources are limited, and energy spent on one is perhaps unavailable for the other (Heaton et al., 2016; van Noordwijk \& de Jong, 1986). Alternately, the fungal mycelium is a network of feeding cells that capture resources and the fungus could only invest in mycelium till it covers the entire resource patch and then reproduce. In such a case, the two stages growth and reproduction are sequential events and may not be in conflict. We were interested in testing the relationship between growth rate and asexual spore yield during adaptation to the laboratory. Natural isolates of filamentous fungi may grow faster when adapting to the laboratory environment by reducing the production of costly extracellular enzymes needed for the breakdown of complex carbohydrates (Zheng et al., 2020). Alternatively, we tested if the initial position of the genotype affected the trajectory of evolution (figure 2.3A). To test these alternate ideas, we set up a short evolution experiment in the laboratory. We tested for possible trade-offs between growth rate and asexual spore production due to short-term adaptive constraints. Such trade-offs could be due to antagonistic pleiotropy of mutations selected in the same environment or due to mutation accumulation.

\section{Materials and methods}

\section{Strains used}

Conidiospore samples of fifteen natural isolates of $A$. nidulans (strains 700,701 , $702,703,704,707,710,711,713,715,717,722,723,726,727$ from the strain collection, Laboratory of Genetics, Wageningen University), isolated in 1992 from various parts of Birmingham, Pembrokeshire and Cardiganshire, UK (Coenen et al., 1996), were inoculated in $30 \mathrm{~mL}$ flasks containing complete agar medium and incubated at $37^{\circ} \mathrm{C}$ for six days. The spores were harvested by adding a few $3 \mathrm{~mm}$ glass beads and $5 \mathrm{~mL}$ of $0.8 \% \mathrm{NaCl}+0.005 \%$ tween and shaken for $10 \mathrm{~min}$ at 200 $\mathrm{rpm}$. The spore suspension was filtered through a sterile funnel with glass wool. A part (500 $\mathrm{\mu l}$ ) of this suspension was mixed in a 1:1 ratio with glycerol/neo-peptone (2:1) and stored at $-70^{\circ} \mathrm{C}$ as a stock.

\section{Media composition}

Complete medium (CM): For $1000 \mathrm{ml}$, add $2 \mathrm{~g}$ peptone, $1 \mathrm{~g}$ yeast extract, $1 \mathrm{~g}$ casamino acid, $6.0 \mathrm{~g}$ of $\mathrm{NaNO}_{3}, 1.5 \mathrm{~g}$ of $\mathrm{KH}_{2} \mathrm{PO}_{4}, 0.5 \mathrm{~g}$ of $\mathrm{MgSO}_{4} .7 \mathrm{H}_{2} \mathrm{O}, 0.5 \mathrm{~g}$ of KCl, $10 \mathrm{mg}$ of $\mathrm{FeSO}_{4}, \mathrm{ZnSO}_{4}, \mathrm{MnCl}_{2}$ and $\mathrm{CuSO}_{4}$ and $15 \mathrm{~g}$ agar. Adjust $\mathrm{pH}$ to 5.8 with 1 $\mathrm{M} \mathrm{NaOH}$. After autoclaving, add $1 \mathrm{ml}$ of vitamin solution $(0.01 \mathrm{~g}$ each of biotin, pyridoxine, thiamine, riboflavin, p-aminobenzoic acid, nicotinic acid in $100 \mathrm{ml}$ water). As a carbon source add $0.1 \%$ or $1 \%$ glucose. 
Minimal medium (MM): For $1000 \mathrm{ml}$, add $6.0 \mathrm{~g}$ of $\mathrm{NaNO}_{3}, 1.5 \mathrm{~g}$ of $\mathrm{KH}_{2} \mathrm{PO}_{4}, 0.5 \mathrm{~g}$ of $\mathrm{MgSO}_{4} \cdot 7 \mathrm{H}_{2} \mathrm{O}, 0.5 \mathrm{~g}$ of KCl, $10 \mathrm{mg}$ of $\mathrm{FeSO}_{4}, \mathrm{ZnSO}_{4}, \mathrm{MnCl}_{2}$ and CuSO ${ }_{4}$. Adjust $\mathrm{pH}$ to 5.8 with $1 \mathrm{M} \mathrm{NaOH}$. As a carbon source add $0.1 \%$ or $1 \%$ glucose.

\section{Mycelial growth rate (MGR), spore yield, and spore density}

For measuring MGR and spore yield, $10 \mu$ of the filtered spore suspension was spot-inoculated onto $92 \mathrm{~mm}$ Petri plates with $20 \mathrm{~mL}$ medium (MM and CM and only MM for the evolution experiment). The MGR was determined by measuring the diameter of each colony in two perpendicular axes and averaged and divided by the incubation time of four days (Bruggeman et al., 2003). To quantify spore yield, the spores were harvested from each colony by adding $5 \mathrm{ml}$ of saline-tween $(0.005 \%)$ onto the plates and scraping off the spores using a metal Drigalsky spatula. The spore suspension was collected, diluted, and counted using a CASY TT cell counter and analyzer (OMNI Life Sciences, Bremen, Germany with a $45 \mu \mathrm{m}$ capillary). Spore density was measured by dividing the total number of spores by the colony surface area.

To measure the effect of environmental condition on growth rate and spore yield, all fifteen natural isolates were cultured on four different solid media with $1.5 \%$ agar (CM $+0.1 \%$ glucose, $C M+1 \%$ glucose, $M M+0.1 \%$ glucose, $M M+1 \%$ glucose) and incubated at $37^{\circ} \mathrm{C}$ for five days. After incubation, MGR and spore yield was determined as above. To quantify trade-offs in the short-term evolution experiment (see below), the ancestors and the evolved populations from the evolution experiment were cultured on evolution medium ( $M M+1 \%$ glucose) and MGR, spore yield, and spore density measured were as above.

\section{Evolution experiment}

Six of the natural $A$. nidulans strains were chosen (see Results) for the evolution experiment and prepared as follows. Spore suspensions from each pre-grown culture were diluted and plated on CM agar plates with $1 \%$ glucose to isolate genetically pure single-spore colonies. Six different colonies were picked from each strain, and some spores were transferred to fresh plates to isolate single colonies. After that, the spores from three independently isolated colonies per strain were used to inoculate fresh plates to get pure cultures and spores stored as ancestors of the evolution experiment. The design of the evolution experiment was as follows: six replicate populations were started for each strain and spotinoculated on $92 \mathrm{~mm}$ Petri plates with $20 \mathrm{ml} \mathrm{MM+1 \%} \mathrm{glucose,} \mathrm{hereafter} \mathrm{referred}$ to as evolution medium. Since we had only three independent colonies as ancestors, each ancestor started two populations of the evolution experiment. The populations were incubated at $37^{\circ} \mathrm{C}$ for four days. After four days of incubation, 
the plates were kept in a cold room overnight. Spores were harvested by adding $5 \mathrm{ml}$ of $0.8 \% \mathrm{NaCl}+0.005 \%$ tween to each Petri plate (Schoustra et al., 2005), scraping off the spores with a metal spreader, transferring the spore suspension to $15 \mathrm{ml}$ tubes. A few $3 \mathrm{~mm}$ glass beads were added, and the suspensions were vortexed to break conidial chains. $0.1 \%$ of the spore suspension $(5 \mu \mathrm{L})$ was used to inoculate the next cycle on fresh media (Schoustra et al., 2005). $500 \mu$ of each sample was mixed with $2 x$ glycerol/neo-peptone and stored as living fossils in a $-70^{\circ} \mathrm{C}$ freezer in duplicates.

\section{Results}

\section{Effect of the environment on life-history traits}

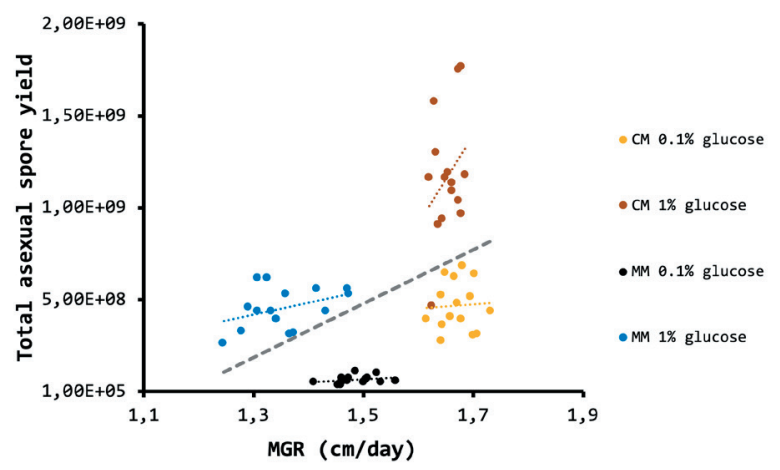

Figure 2.1: Effect of nutrient conditions on life-history traits. Growth rate and spore yield in different nutrient conditions of 15 wild isolates of $A$. nidulans are shown. Each data point is an average of three replicate measurements along with the trend lines within treatments and the grey dashed line shows the trend across treatments.

We first asked whether a trade-off between mycelium growth rate and asexual spore production in $A$. nidulans would be so fundamental that it would be apparent among wild isolates tested in a standard laboratory environment. Fifteen wild isolates of $A$. nidulans isolated from soil samples of various parts of UK were grown on four different media conditions. We used a nutrient-poor medium, minimal medium (MM) composed of inorganic salts, and a nutrient-rich medium, complete medium (CM) containing complex organic compounds like amino acids, nucleotides and vitamins in addition to the salts of MM. We supplemented each medium with either a $1 \%$ or $0.1 \%$ glucose to vary energy availability. As can be seen in figure 2.1, the strains had higher MGR and higher spore yield when grown on CM versus MM. Moreover, spore yield also increased with glucose concentration, whereas MGR showed rather an opposite response, with no change in $C M$, but lower MGR values in $\mathrm{MM}$ with high glucose. A multivariate analysis using a generalized linear model shows a significant effect of the richness of the medium on both the MGR and spore 
yield $(p<0.0001)$, and glucose concentration also has a significant effect on MGR $(p<0.0001)$, and on spore yield $(p=0.005)$. The interaction of richness and glucose concentration was significant only for MGR $(p<0.0001)$, as high glucose reduced MGR on MM but had no effect on $C M$, but not spore yield $(p=0.558)$. However, we did not observe a trade-off between growth rate and reproduction between strains within any of the four environments (correlation coefficients ranging from 0.05 to 0.38 , with $p>0.05$ in all cases) (figure 2.1 ).

\section{Experimental evolution with a subset of the isolates}

As the natural isolates did not show a trade-off between mycelium growth rate and spore yield, we then asked whether constraints would affect the short-term evolution of these traits. Six (two from Birmingham, one from Pembrokeshire and three from Cardiganshire) of the fifteen strains were used in a 10-week evolution experiment on a minimal medium with $1 \%$ glucose since this environment showed maximum variation among strains in MGR and spore yield. The six strains used in the evolution experiment were chosen because they spanned the entire range of MGR and spore yield values. We tested for trade-offs in MGR and spore yield in two ways. First, we asked whether independently evolved populations would show a negative correlation between both traits, as adaptation might lead independently evolving populations to distinct places on the presumed trade-off line. Second, we tested if the initial position of the genotype affected the trajectory of evolution (figure 2.3A).

\section{Correlations across the independently evolved populations}

A trade-off could also become apparent when the independently evolved populations would arrive at distinct positions on, or close to, the presumed tradeoff line. A fungal colony can increase spore yield by increasing the surface area for sporulation or increasing the spore density while keeping the surface area small. Hence, we tested for a trade-off between MGR and spore yield and between MGR and spore density across the 36 replicate populations. Figure 2.2A shows the relative MGR versus relative spore yield and 2.2B the relative MGR versus relative spore density (where relative means relative to the ancestral strain) at the final time-point of the evolution experiment. We did not observe a trade-off between growth rate and spore yield or density among the six replicate populations of each of the six isolates (correlation coefficients ranging from 0.47 to -0.47 for MGR and spore yield and from 0.23 to -0.57 for MGR and spore density, $p>0.05$ in all cases). We also did not observe a significant correlation between relative MGR and relative spore yield across the replicate populations. However, we found a negative correlation between mycelium growth rate and spore density across all 36 replicate populations, which approaches significance $(r=-0.31, p=0.065)$. 

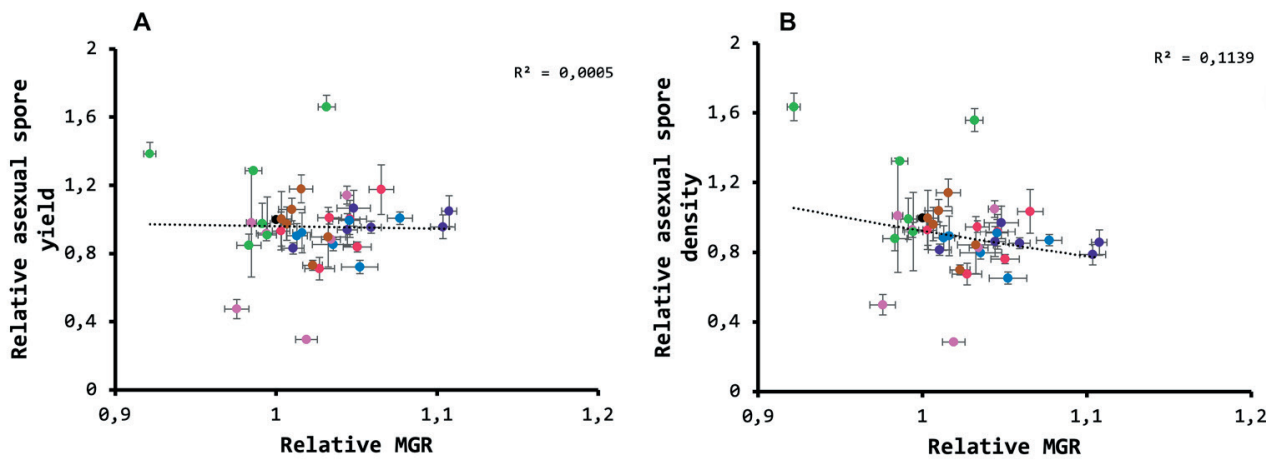

Figure 2.2: Association between mycelium growth rate and spore production among laboratoryevolved populations of $\boldsymbol{A}$. nidulans. (A) Association between relative spore yield and relative mycelium growth rate (MGR) of six replicate populations derived from each of six natural $A$. nidulans isolates after 10 weeks of evolution in the laboratory; (B) Association between relative spore density and relative mycelium growth rate (MGR) of the same 36 evolved populations. Each data point represents the mean of three replicate measurements; error bars are SEM and the six populations derived from the same natural isolate are grouped by one color.

\section{Effect of initial position on the trajectory of evolution}

The strains chosen as ancestors for the evolution experiment represented the whole range of growth rate and spore yield values observed among the 15 natural isolates. We tested if the ancestor's position on the MGR and spore yield axes affected the trajectory of evolution (figure 2.3A). We performed this analysis both at the level of spore yield and spore density (see figure 2.3B and figure 2.3C). As can be seen, adaptation to the laboratory environment was realized by increases of the MGR associated with decreased spore yield in five of the six wild strains, while in the strain with the highest MGR, adaptation involved a slight increase in spore production, associated with a decrease in MGR. If both traits would evolve independently, positive and negative associations between the direction of change in each trait would be equally likely, while we find changes consistent with antagonistic pleiotropy in all six strains (Binomial probability $P=0.5^{6}=0.016$ ). Therefore, although changes in both traits are significant in only one of the six strains after correction for multiple testing (Welch two-sample t-test, $p=0.001$ for MGR and $p=0.0006$ for spore density), the consistent antagonistic direction of change in both traits across the six natural isolates suggests that a growthreproduction trade-off poses adaptive constraints at least during short-term lab evolution. 
A

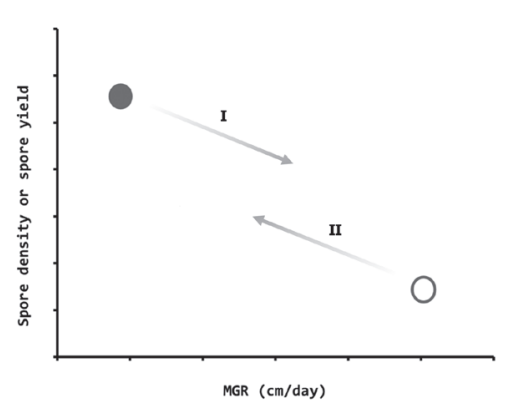

B

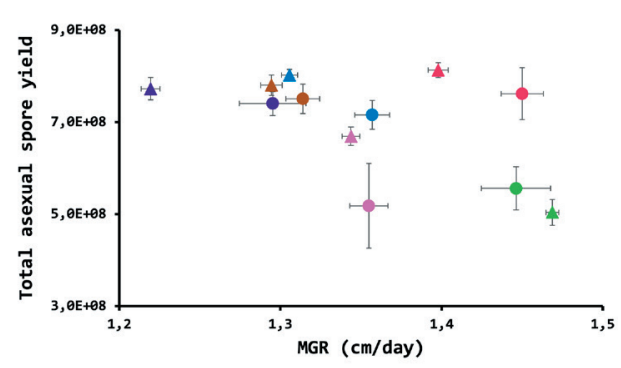

C

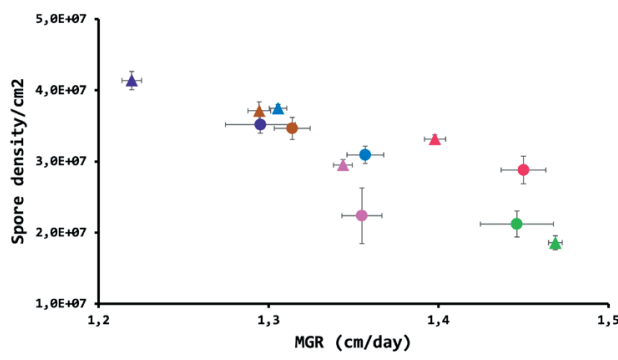

Figure 2.3: Effect of position on the trajectory of evolution. (A) This figure illustrates the different hypothetical scenarios for the direction of adaptation when adaptation is affected by the starting position of the ancestor. I: If the ancestor has low MGR and high spore yield, then the evolved populations might improve their MGR at the cost of their spore yield; II: An ancestor that has high MGR and low spore yield might improve their spore yield at the cost of MGR. (B) Mean MGR and mean spore yield; (C) mean MGR and mean spore density after 10-weeeks of evolution. Each color represents one isolate. The ancestors are represented by triangles and the evolved genotype by circles. Each point represents the mean of 6 replicate populations and the starting and end position of the genotype. Error bars are SEM.

\section{Discussion}

This study was designed to understand the effects of long-term and short-term evolutionary constraint (during laboratory evolution) on growth and reproduction of natural isolates of $A$. nidulans. Like other organisms, fungi are predicted to alter their life-history strategies based on the richness, life span, and the presence of competitors in the niches they inhabit (Gilchrist et al., 2006; Heaton et al., 2016). To understand the effect of local adaptation on growth rate and spore yield, we grew 15 natural isolates of $A$. nidulans on nutrient-poor and nutrient-rich media with high and low glucose. We expected that measuring MGR and spore yield of these strains in the laboratory may reflect trade-offs between these traits if these would have been apparent during adaptive divergence in their natural habitats, despite differences in the actual value of these traits. For example, a study on natural populations of basidiomycete Schizophylum commune showed variation 
in mycelium growth rate and fruiting body production among natural isolates collected from a large patch of soil (Brasier, 1970). Our results did not support such a trade-off in any of the four lab environments, but we saw a positive correlation between the traits measured across environments (figure 2.1). The failure to find a trade-off within an environment in our results may indicate that such a trade-off was unimportant during their independent adaptive histories (Bono et al., 2017). Alternatively, a trade-off was apparent during local adaptation but obscured by differences between lab and natural conditions. Yet another possible explanation may be that $A$. nidulans has evolved to be a generalist, such as found for natural isolates of E. coli and Salmonella enterica (Bronikowski et al., 2001). Bronikowski et al. (2001) tested the effect of temperature on natural isolates of $E$. coli and $S$. enterica isolated from different thermal environments and found that the isolates were thermal generalists and their growth rates were unaffected by changes in their environmental temperature (Bronikowski et al., 2001).

The effect of short-term constraints from a trade-off between growth and reproduction was tested by conducting a short evolution experiment in the laboratory. The evolution conditions were chosen based on the data from the growth on different media: we chose strains and a condition representing maximum variation in growth and reproduction. When comparing fungal colony size and spore production, three scenarios are possible: i) Colony size and spore yield might be directly proportional to each other as observed in a study with Aspergillus niger (De Visser et al., 1997); ii) Colony size and spore yield might be inversely proportional to each other (Schmit, 2002; Schoustra \& Punzalan, 2012), iii) These two parameters might vary independently (Anderson et al., 2019). To ensure that our analysis is unbiased based on the possibilities mentioned above, we compared total spore yield and spore density with mycelial growth rate.

We measured the correlation between growth rate, spore yield, and spore density in the independently evolved populations at the final time point. We did not find a negative correlation between growth rate and spore yield (figure 2.2A). However, we found a negative correlation between growth rate and spore density that approached significance when the independently evolved populations were compared at the final time-point (figure 2.2B). If growth and spore yield were uncorrelated, a fungal colony produced a fixed number of spores under a specific environmental condition. In such a case, an increase in the colony size will negatively correlate with spore density but not with total spore yield. A similar negative correlation between MGR and spore density was observed in another study with a laboratory strain of $A$. nidulans (Schoustra \& Punzalan, 2012). In another filamentous fungus, Neurospora crassa, Anderson et al. (2019) evolved the 
natural isolates in the laboratory for improvement in either growth rate or spore yield and found that selection on either of these traits did not affect the other. They concluded that in $N$. crassa, both growth rate and spore yield are independent traits (Anderson et al., 2019).

Pfeiffer et al. (2001) postulated that a trade-off exists between the rate and yield of ATP production in heterotrophic organisms. Novak et al. (2006) hypothesized that selection would favor traits that would increase growth rate, hence the rate of ATP production, when organisms adapt to growth on limiting nutrients. They tested for the existence of a trade-off between growth rate and yield in 12 populations from the $E$. coli long-term evolution experiment (Lenski et al., 1991). The authors tested for a trade-off between growth rate and yield across evolutionary time, between independently evolved replicate populations, and clones within the evolved populations. They detected a trade-off between rate and yield among clones for three of the four populations tested, but not across the 12 populations (Novak et al., 2006). The design of our evolution experiment was not aimed at selecting for either growth or reproduction. However, a previous study by Schoustra et al. (2005) showed that the protocol used resulted in a rapid increase in growth rate when the antifungal resistant strain, with a severe cost of resistance was adapting to growth in the absence of the toxin by compensatory mutations.

Strains chosen as ancestors for the evolution experiment were the ones that were on the extreme ends of the growth and spore yield spectrum and a few in between. Strains at the extreme ends were selected to see if their position at the start of the experiment particularly affected the trajectory of evolution (figure 2.3A). Alternatively, the constraint between growth and reproduction may cause a negative correlation between the two traits irrespective of their starting position, or if the six natural isolates would be maladapted to the laboratory environment and they are lower on the growth and spore yield axis than they would achieve when they are on the trade-off line, then both traits would improve (Novak et al., 2006). The evolutionary trajectories of figure 2.3B and figure 2.3C showed that five of the six populations improved their growth rate with or without a significant decrease in spore yield. Among these six isolates, only one isolate showed a significant reduction in spore density with an increase in MGR across all six evolved replicates. Natural isolates of filamentous fungi are known to grow faster by reducing the production of costly extracellular enzymes needed for the breakdown of complex carbohydrates when adapting to the laboratory environment (Zheng et al., 2020). Lustenhouwer et al. (2020) conducted a meta-analysis on the correlations between fungal life-history traits of wood decomposing fungi. They found a negative correlation between hyphal density and extension rate, which reflect alternate 
growth strategies, and competitive ability and tolerance to environmental variation, presumably reflecting differences in ecological performance strategies. The fast growers were competitively dominant, while the slow growers were more stress-tolerant. This trade-off between traits is reflective of their spatial distribution and local environment-dependent modification of life-history traits (Lustenhouwer et al., 2020). Natural isolates of Podospora anserina, a senescing pseudo-unitary fungus show variation higher growth rate (from no change to a 5 -fold) on low concentration ( $0.1 \%$ ) of glucose. This variation is associated with the presence or absence of a mitochondrial plasmid (Maas et al., 2004). The variation in fitness related traits shown by natural isolates of fungi result from environmental effects or due to genetic elements like plasmids.

Although we saw variation in MGR and spore yield of natural $A$. nidulans isolates across environments, we did not see any correlation between these traits across these isolates. The evolution experiment suggests that a negative correlation between changes in MGR and spore density may exist after short-term evolution, but not between MGR and total spore yield, indicating the absence of a strong constraint between these traits. Only one strain increased in MGR during evolution at the expense of spore density, and three strains only improved in MGR without a significant change in spore yield or density. While theoretically trade-offs are expected to drive improvements in fitness-related traits, at least in wild isolates of $A$. nidulans, we did not see its effect on either long- or short-time scales in the conditions we tested. There might still be a trade-off between lifehistory traits that we have not tested, such as between growth and the rate and frequency of sexual reproduction. The natural isolates we used in our study will likely have experienced environments during their adaptation that were diverse and very different from the ones we used in our tests, which may have obscured any effects from a trade-off between growth and reproduction (Velicer \& Lenski, 2007). The position of the genotypes relative to a possible trade-off line will determine whether we observe a trade-off or not. If the genotypes are far below from the trade-off line, adaptation will result in improvements in both growth and reproduction so no trade-off will be visible. 


$$
3
$$




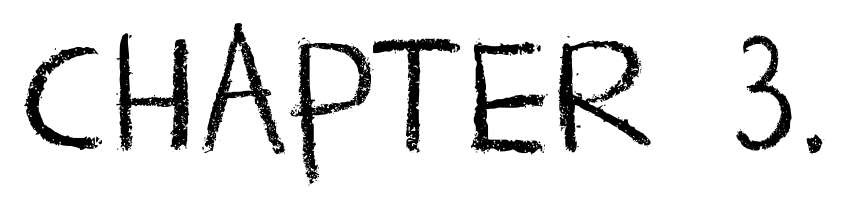

\section{Adaptive dynamics of the fungus Aspergillus nidulans during a one-year evolution experiment}

Krithi Nandimath ${ }^{1,2}$, Mirre Klatter ${ }^{1}$, Marijke Slakhorst ${ }^{1}$, Sijmen E. Schoustra ${ }^{1}$, Alfons J. M. Debets ${ }^{1}$, Vidyanand Nanjundiah ${ }^{2}$, Duur K. Aanen ${ }^{1}$ and J. Arjan G. M. de Visser ${ }^{1}$ 


\section{Abstract}

Multicellular fungi can be an excellent model for experimental evolution. They combine the complexity of multicellular eukaryotes with short generation times and ease of manipulation offered by single-celled organisms. Yet, their life cycle is more complex, including the development of somatic and reproductive tissues, which raises the question of how these different stages contribute to adaptation in a novel environment. To test this, we performed a one-year evolution experiment with the ascomycete fungus Aspergillus nidulans, using weekly transfer of $1 \%$ of the asexual spores, and analyzed changes in fitness and its components. Mean mycelial growth rate did not change significantly, while, surprisingly, asexual spore yield declined approximately three-fold in all 12 parallel populations. Additionally, competitive fitness against the ancestor did not show improvement in most evolved populations. However, fitness measurements for two populations using recent predecessors as competitors, revealed that non-transitive fitness interactions may explain the lack of increased fitness relative to the ancestor. A closer look at variation within populations showed at least two morphotypes in all the populations. One morphotype differed from the ancestral phenotype, having a cottony phenotype. Our results show that adaptation of a filamentous fungus to a simple structured environment is complex and not due to increases in conventional fitness measures, such as mycelium growth rate, spore production or competitive fitness relative to the ancestor. Rather, adaptation involved interactions among different genotypes present within populations. 


\section{Introduction}

Several studies published in the 1950s (Atwood et al., 1951; Bryson \& Szybalski, 1952; Novick \& Szilard, 1950) are the forerunners of microbial experimental evolution. Their common theme was understanding the process of adaptive evolution via selective sweeps of favorable mutants (J. Adams \& Rosenzweig, 2014). Only decades later, microbes, with their short generation times, ease of experimentation, and the possibility of storage as 'living fossils' in the freezer, have become popular systems to address questions in evolutionary biology in realtime (Elena \& Lenski, 2003).

The early days of experimental evolution saw single-celled organisms evolving in unstructured environments and competition among genotypes occurring on a population-wide scale (Dykhuizen \& Hartl, 1983). A structured environment provides an opportunity for environmental heterogeneity, the formation of niches, and the evolution of specialists, limiting competition to local spatial scales. Rates of invasion of beneficial mutants with limited dispersal are constrained as the mutant is competing locally, mostly with clone mates, and modest migrations do not erode the effects of spatial structure within populations (France \& Forney, 2019). The rate of adaptation is slower due to the fixation of smaller beneficial mutations (Habets et al., 2006). Fitness benefits of adaptive mutants are smaller in a structured environment when compared to an unstructured environment, because most competition happens among clone mates, making improvements in dispersal or interference competition more effective (Habets et al., 2007).

For most asexual unicellular organisms, competitive fitness, often quantified in head-to-head competition assays with the ancestor, integrates all relevant fitness components (Elena \& Lenski, 2003). Adaptation in an asexual population may proceed by the continuous replacement of one genotype by a fitter genotype (Atwood et al., 1951). But several exceptions exist, with multiple examples of asexual populations that have stable co-existence of more than one genotype in their populations (Good et al., 2017; Habets et al., 2006; Helling et al., 1987; Rainey \& Travisano, 1998; Rosenzweig et al., 1994). Interactions like metabolic crossfeeding, resource competition, and interference competition between genotypes can lead to negative frequency dependence of fitness interactions, causing multiple genotypes' to co-exist stably (Cvijović et al., 2018).

Over the years, filamentous fungi have become popular model systems for experimental evolution and they combine the advantages mentioned above of unicellular organisms with the complexity of multicellular growth (Fisher \& 
Lang, 2016). Studies have used multicellular fungi to address a range of different topics, including the role of compensatory mutations to antimicrobial resistance (Schoustra et al., 2006; Zhang et al., 2015), effects of relatedness in maintaining multicellular cooperation (Bastiaans et al., 2016), reproductive isolation and speciation (Dettman et al., 2008), effects of ploidy and nuclear co-adaptation (Clark \& Anderson, 2004) and the role of sexual selection (Nieuwenhuis \& Aanen, 2018).

Here, we describe the results of a one-year evolution experiment with the multicellular fungus Aspergillus nidulans. A. nidulans starts as a single spore with a single haploid nucleus that germinates to form a network of hyphal cells with many nuclei, the mycelium. After a few days of growth, the mycelium starts forming asexual spores or conidia (Pontecorvo,1953). The design of our "A. nidulans Short-term Evolution eXperiment" (ASEX) was inspired by the longterm evolution experiment with the bacterium Escherichia coli (LTEE) by Richard Lenski and colleagues (Lenski et al., 1991). Twelve replicate populations evolved on synthetic minimal glucose agar medium for one year by weekly transfer of $1 \%$ of the produced asexual spores dispersed on fresh medium. Thus, there was an alternation between mycelium growth and asexual sporulation in a structured environment and mixing during transfer, and sexual reproduction played no role. The main aim of the experiment was to understand the role of mycelial growth rate (MGR) and asexual reproduction (asexual spore yield), including possible trade-offs among them, and the nature of competitive ability of $A$. nidulans when adapting in a simple structured environment, alternating with dispersal.

We tested for a possible trade-off between MGR and spore yield in two ways. First, we quantified the changes in MGR and spore yield over evolutionary time. Here, a trade-off can be manifested as an improvement in one trait with a concomitant decrease in another trait over time. Second, we measured the correlation between the two traits in the independently evolved populations at the final time-point. Here, a trade-off would be manifested if the populations take different routes during adaptation and lead them to distinct points on, or close to, a trade-off line. However, if the populations are maladapted to the evolutionary environment, we might observe an improvement in fitness traits (Novak et al., 2006). We have also measured changes in competitive fitness of the populations in head-to-head competitions with the ancestor and recent predecessors to measure adaptation involving other fitness components. 


\section{Materials and methods}

\section{Experimental evolution set up}

Aspergillus nidulans WG649 and WG650 were used to start the ancestral population. The strains are isogenic derived from prototrophic strain WG547, differing in nitrate non-utilizing mutations and spore color. WG649 has wild-type green color spores and a nia mutation, and WG650 has yellow spores and a cnx mutation. These strains were used to start 12 replicate populations- 6 with WG649, hereafter referred to as N1-N6 and 6 with WG650, henceforth referred to as Y1-Y6. The strains were inoculated in $30 \mathrm{ml}$ glass bottles with $10 \mathrm{ml}$ of solid minimal media (MM) (For 1000 $\mathrm{ml}$, add $6.0 \mathrm{~g}$ of $\mathrm{NaNO}_{3}, 1.5 \mathrm{~g}$ of $\mathrm{KH}_{2} \mathrm{PO}_{4}, 0.5 \mathrm{~g}$ of $\mathrm{MgSO}_{4} .7 \mathrm{H}_{2} \mathrm{O}, 0.5 \mathrm{~g}$ of KCl, $10 \mathrm{mg}$ of $\mathrm{FeSO}_{4}, \mathrm{ZnSO}_{4}, \mathrm{MnCl}_{2}$ and $\mathrm{CuSO}_{4}$. Adjust $\mathrm{pH}$ to 5.8 with $1 \mathrm{M} \mathrm{NaOH}$ ) (Pontecorvo, 1953), supplemented with $0.1 \%$ glucose and $5 \mathrm{mM}$ urea, hereafter referred to as evolution medium. The populations were started by evenly spreading $50 \mu \mathrm{l}$ of a spore suspension containing $10^{6}$ spores on the surface of the agar. The bottles were incubated at $37^{\circ} \mathrm{C}$ in a static incubator for seven days with loose caps. After seven days, spores were harvested by adding $5 \mathrm{ml}$ of saline containing $0.05 \%$ tween and the addition of a few $3 \mathrm{~mm}$ glass beads. The bottles were placed in a horizontal shaker and shaken for $10 \mathrm{~min}$ at $200 \mathrm{rpm}$. The spore suspensions were filtered through a glass funnel fitted with glass wool. A sample of $50 \mu \mathrm{l}(1 \%)$ of the filtrate was used to start the next culture. The spore suspension was spread over the surface of the media by shaking manually. A $1 \mathrm{ml}$ aliquot of the sample was stored in a $-80^{\circ} \mathrm{C}$ freezer with the addition of glycerol/peptone (29\%:0.67\%) as a 'living fossil.' This process was repeated every week for one year (figure 3.1). Weekly transfers of green (WG649) and yellow populations (WG650) were alternated to facilitate the detection of cross-contamination. If contamination with spores from a different spore color occurs and reaches frequencies of $\sim 1 \%$ or higher in the invaded population, differently-colored colonies would have been detected in controls (i.e., yellow colonies in a wild-type evolving strain or vice versa). No crosscontamination was observed in our populations.

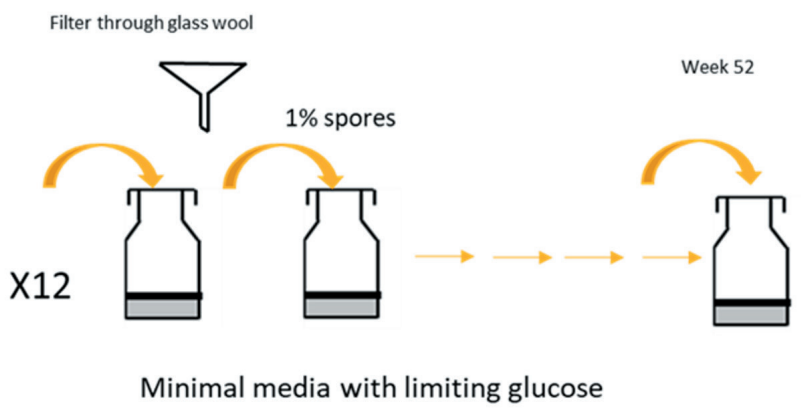

Figure 3-1: The design of the ASEX experiment. 


\section{Quantifying fitness}

\section{Measurements of fitness components}

Mycelial growth rate (MGR) To measure MGR, $20 \mathrm{ml}$ of evolution medium was pipetted into $90 \mathrm{~mm}$ Petri plates and dried on the bench overnight. $3 \mu \mathrm{l}$ of spore suspension was added to the center of the Petri plate. Each strain was inoculated in triplicate. The spore suspension was allowed to be absorbed into the media before the plates were inverted and incubated at $37^{\circ} \mathrm{C}$ for five days. After five days of incubation, the diameter of the colony was measured in two perpendicular directions.

Total asexual spore yield $10 \mathrm{ml}$ of evolution medium was pipetted into $30 \mathrm{ml}$ glass bottles and allowed to stand on the bench overnight. The next day, $1 \%$ of the spore suspension was inoculated by spreading on the surface of the media by shaking the bottle manually. The bottles were incubated at $37^{\circ} \mathrm{C}$ for seven days. The surface area available for all strains to grow is small in the bottle, and the mycelium covers the entire surface of the agar. The spores were harvested like during the evolution experiment mentioned above. The spores were counted using a Casy TT particle counter (OMNI Life Science \& Co KG, Germany) following the manufacture's protocol for counting fungal spores.

\section{Competitive fitness assays}

\section{Acclimatization of the populations or clones}

Before the competitions, populations/strains were first grown for a week on the evolutionary medium to remove the effects of the glycerol in which they are stored and then grown for another week to condition the strains to the evolutionary environment. The spores were counted with the Casy TT particle counter.

\section{i. Evolved population vs. ancestor}

The twelve populations and the two ancestors were acclimatized, as mentioned above. After acclimatization, the spores were harvested identical to the evolution experiment and counted using the Casy TT particle counter. Spore samples were then mixed in a 1:1 ratio of the ancestor (yellow): evolved (green) and vice-versa at a spore density of $2 \times 10^{6}$ spores $/ \mathrm{ml}$. $100 \mu \mathrm{l}$ of this mixture was inoculated on fresh medium in the evolutionary environment and incubated with loose caps for seven days at $37^{\circ} \mathrm{C}$ in the dark. The ratios of both competitors were determined both before and after the competition. The ratios were determined by inoculating counting plates (complete medium with $0.05 \%$ tween $(\mathrm{v} / \mathrm{v})$ ) and counting $50-100$ green and yellow colonies per individual measurement. With these ratios, the 
following formula was used to calculate the selection rate constant, $r$, as a measure for the difference in fitness of the two strains (Lenski et al., 1991). Each assay was performed in triplicates.

$$
\begin{array}{r}
r=\ln \left(\frac{\# \text { of evolved colonies after competition }}{\# \text { of ancestral colonies after competition }}\right) \\
-\ln \left(\frac{\# \text { of evolved colonies before competition }}{\# \text { of ancestral colonies before competition }}\right)
\end{array}
$$

\section{ii. Transitivity of competitive fitness}

a. Against ancestor Populations N1 and N3 were acclimatized and treated as mentioned above. The spore samples were counted using the Coulter counter.

\section{b. Against recent predecessor for non-transitive fitness interactions}

The fitness of both evolved populations (N1 and N3) was determined through direct competition of each time-point sample against a recent predecessor. To compare evolved populations with a recent predecessor, yellow-colored mutants were selected for every other time point chosen after treating spores with a low dose of UV light ( $42 \mathrm{erg} / \mathrm{mm}^{2} / \mathrm{s}$ ) for 10 seconds. A mycelium growth rate measurement was used to select the strain with the least adverse effects of UV mutagenesis. The color mutants were competing against a population sample from four weeks earlier and one from four weeks later, as well as the original (i.e., with green spores) wild-type population from the same time point as a reference. Each assay was performed in triplicate for every time point (14 in total with 4-week intervals) of the two evolved populations. A visual representation of these competitions can be found in figure 3.3C. The fitness difference between the color mutants and originally evolved populations they were picked from was used to correct for the marker-mutation effect, assuming additive effects.

\section{Results}

\section{Test of MGR-spore yield trade-off over evolutionary time}

The mycelium of a filamentous fungus is used for searching, assimilating and transporting food and it is also serves as an organ for supporting reproductive propagules (spores). Therefore, one can assume that a larger mycelium would provide a larger surface area for sporulation and improvements in MGR might be selected and both the traits might be positively correlated. To test for the correlation between MGR and spore yield, we assayed for changes in two characteristics, mycelial growth rate (MGR) and spore yield from the ancestor and all 12 populations, at 10-week intervals. A trade-off would be visible if improvements in MGR would be associated with a decrease in spore yield or vice-versa. If the ancestral strain is far- 
off from the trade-off line, we might see improvements in both the traits (Novak et al., 2006). On average, the 12 populations showed a modest but significant increase in MGR of $\sim 4 \%$ in the first ten weeks (Welch two-sample t-test, $t=-4.214$, $p=0.0014$ ). The initial increase was followed by a slow decline, reaching a net change of $\sim 2 \%$ relative to the ancestor by the end of the experiment, which was not significant (Welch two-sample t-test, $t=-1.41, p=0.19$; figure 3.2A). Changes in MGR varied across populations and did not show a consistent pattern. The total asexual spore yield of the populations was also measured at 10-week intervals, as shown in figure 3.2B. The 12 populations showed a clear, gradual decrease in spore yield, resulting in a highly significant reduction of more than $50 \%$ by the end of the experiment (Welch two-sample t-test, $t=23.88, p=0.0001$ ). The reduction in spore yield was consistent across populations, but individual populations showed considerable variation during the initial weeks of evolution. We find no support for a trade-off between MGR and asexual spore yield.

\section{Test of MGR-spore yield trade-off among evolved populations}

We tested for the correlation between MGR and spore yield between the 12 independently evolved populations. The figure 3.2C shows the average MGR and average total spore yield of the populations at week 50 . We did not observe a negative correlation between these traits. Instead, we observed a positive, statistically non-significant correlation between the two traits $(r=0.52, d f=10$, $p=0.076$ ). Hence, we found no evidence for a trade-off between MGR and spore yield among the evolved populations from the final time point.

A

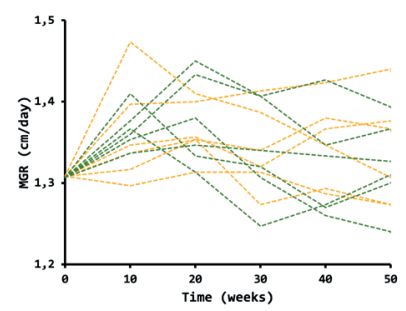

B

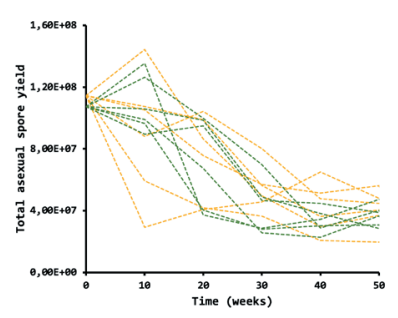

C

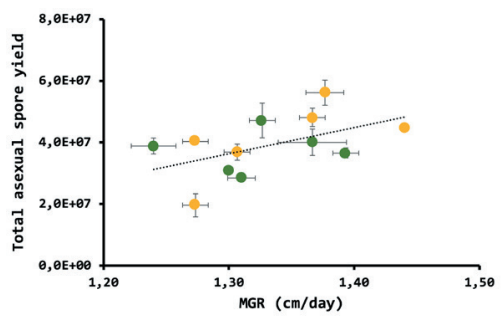

Figure 3.2: Changes in MGR and asexual spore yield over time and among replicate populations at the final time-point. (A): Mycelial growth rate and (B) total asexual spore yield at 10-week intervals. The dashed lines represent the mean value for each of the 12 populations at 10-week intervals. (C) Association between growth rate and spore production across independently evolved populations at week-50. Each value is the mean of three replicate measurements of a single population. Error bars are SEM. 


\section{Competitive fitness}

Since the evolved populations showed no increase in MGR together with a reduced spore yield, we performed competition experiments between population samples from the final time point of the experiment and the ancestral strain with the other spore-color marker to test for adaptation involving other fitness components. To our surprise, the evolved populations did not improve their competitive fitness relative to the ancestor (figure 3.3A; t-test, p>0.05 after correcting for multiple testing using the serial Bonferroni method). The general lack of improvement in competitive fitness prompted us to analyze the dynamics of adaptation. We performed 14 competition experiments with the ancestor and population samples taken from two populations, N1 and N3. The two populations showed similar dynamics of fitness changes: relative fitness increased during the first few weeks of evolution, up to week 16 for N1 and week 12 for N3, followed by a leveling off and then a decline. The second peak of fitness increased during the last few weeks, as seen in the dashed lines in figure 3.3B.

\section{Transitivity of fitness interactions}

The early rapid increase in fitness relative to the ancestor followed by a later decrease could be due to non-transitive fitness interactions resulting from changes in the selective environment caused by the evolving populations. To test for non-transitive fitness interactions, we set up 20 competition experiments as depicted in figure 3.3C. The sum of fitness increments measured in competition with the recent predecessor showed no longer a decline in fitness (solid lines in figure 3.3B). The fitness of $\mathrm{N} 1$ in competition with its recent predecessor is lower than its fitness in competition with the ancestor up to week 40 (see solid line in figure 3.3B. 
A

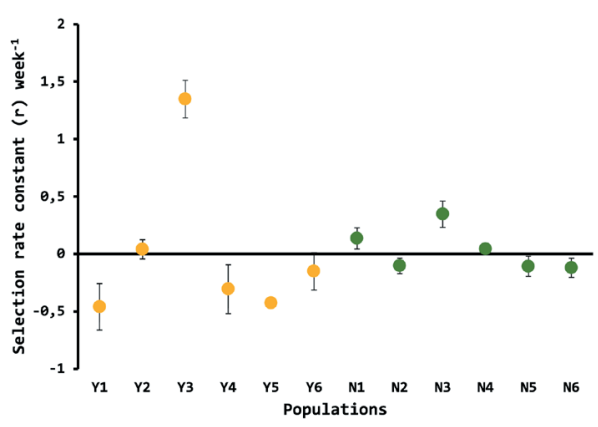

B

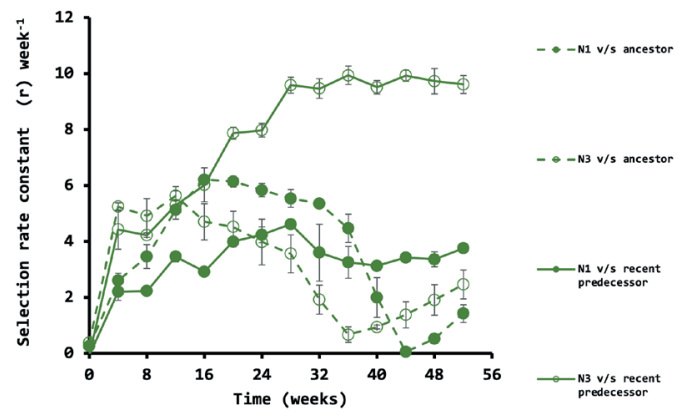

C

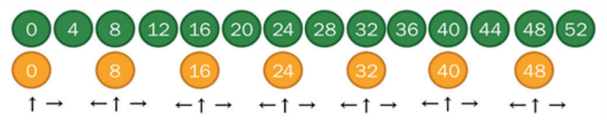

Figure 3.3: Competitive fitness of evolved ASEX populations. (A) Selection rate constant of the evolved populations at week 52 in competition with the ancestor. Each data point is the average of fitness of one of the 12 evolved populations in competition with the ancestor of the opposite spore color. The experiment was carried out in triplicate and the error bars are SEM. (B) Selection rate constant of samples from populations $\mathrm{N} 1$ and N3, as measured by competition with the ancestor (dashed lines) and recent predecessor (solid lines). Each data point of the calculated selection rate constant is the average of three (against ancestor) or two (against recent predecessor) biological replicate measurements. Error bars are SEM. (C) A visual representation of competitions that were performed. Colour mutants were selected for every other time point, which were used in competition with a population sample from the time point before it $(\leftarrow)$, itself ( $\uparrow$, to estimate the effect of the spore-colour mutation), and the time point after it $(\rightarrow)$. Numbers indicate weeks from the start of the evolution experiment.

\section{Polymorphism in the populations}

The lack of improvement in competitive fitness and its presumed components, together with the observed non-transitive fitness interactions, hint at the possibility of complex dynamics between different morphotypes within our populations. Indeed, the competition assays had revealed the presence of at least two different colony morphotypes in the populations. One morphotype resembled the ancestor, henceforth indicated as ancestor-like (AL). The second morphotype produced seemingly fewer spores, and the colony was cottony, white, and fluffy in appearance, hereafter indicated as fluffy-like (FL) (figure 3.4). FL appears as early as week 10 in some populations, is present in all the populations by week 20, and remains present until the end of evolution in eleven populations (figure 3.5). The time when the competitive fitness of populations N1 and N3 with the ancestor decreased (figure 3.3B dashed line) coincides with the time when FL invades in these two populations (figure 3.5). We compared MGR and spore yield of the two morphotypes from the final time point across populations, but did not find a significant difference (paired t-test, $p>0.05$ for both MGR and spore yield) in the MGR and spore yield of FL and AL from the final time-point (figure 3.6A). 


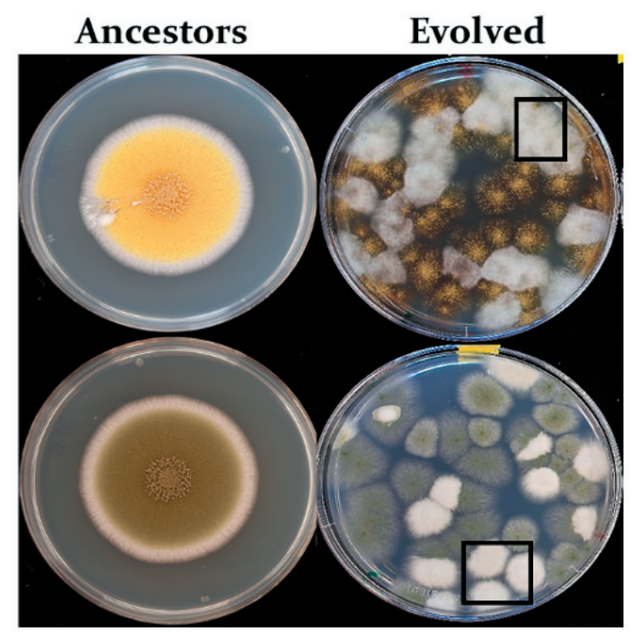

Figure 3.4: Images of the ancestor and two evolved populations. Both spore-colored ancestors are on the left and representative images of a yellow and green evolved population from the final timepoint are on the right. All populations are plated on complete medium for visualization purposes only (to amplify the differences in their phenotype). White colonies on the right panel are the fluffy-like (FL) morphs indicated by the black box.

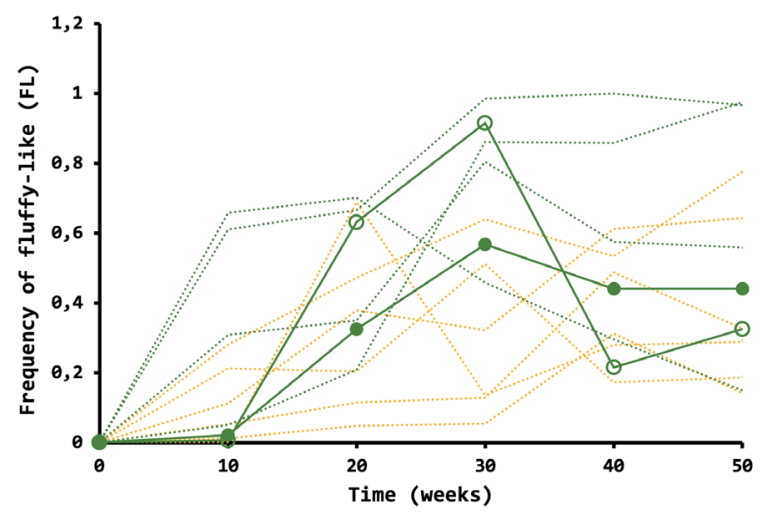

Figure 3.5: Frequency of FL over time. Frequency of FL at 10-week intervals. The dashed line shows the FL frequency of the 10 replicate populations and the remaining two populations used for nontransitive fitness assay namely N1 (closed circle) and N3 (open circle) are shown in solid lines. 
A

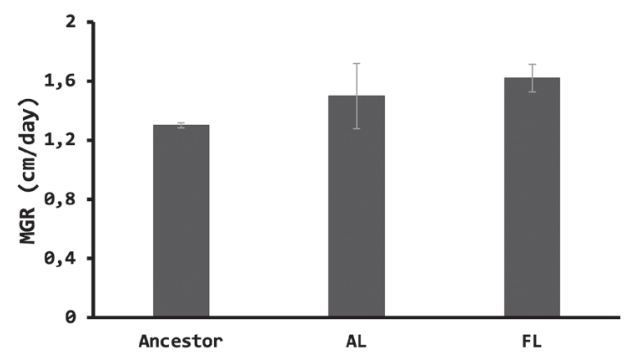

B

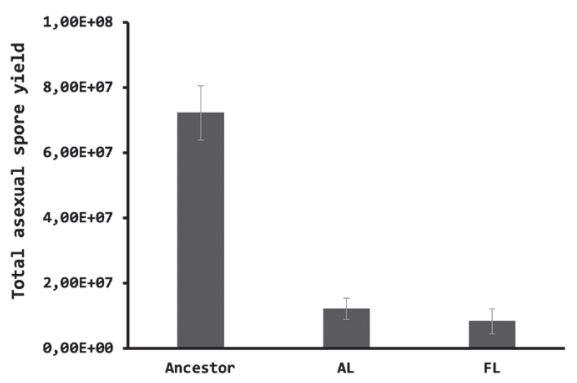

Figure 3.6: Fitness components of AL and FL at the final time-point. (A) MGR of the ancestor and the grand mean MGR for AL and FL from seven populations where the polymorphism persisted until the final time-point. Error bars are standard deviation. (B) Total asexual spore yield of the ancestor and the grand mean spore yield for $A L$ and FL from the same seven populations at the final time-point. Error bars are standard deviation.

\section{Discussion}

The work aimed to understand the role of two life-history traits, i.e., mycelium growth and asexual reproduction, as well as competitive fitness, during adaptation of $A$. nidulans to a simple structured environment. With these objectives and inspiration from the LTEE populations (Lenski et al., 1991), the ASEX populations were set up. The fungus was evolved in a structured environment to facilitate the completion of its asexual life cycle, and spores were used as propagules to start the next cycle. We monitored changes in mycelium growth rate, asexual spore production and the competitive fitness of the evolved genotypes, to assess adaptation to this novel environment. Since the transfer protocol used asexual spores as propagules for starting new growth cycles, we expected to see an increase in asexual reproduction during adaptation to the evolutionary conditions. We were also interested in understanding the trade-offs between the two lifehistory traits. During growth in limiting nutrients, any mutations that improved growth and (or) reproduction are expected to be selected, as such mutants would be over-represented in the spores founding the next cycle.

When populations are evolving in the presence of limiting nutrients, one may expect a trade-off between growth and reproduction since the amount of available energy is limiting and increased energy investment to one trait will come at the cost of reduced available energy for another trait (van Noordwijk \& de Jong, 1986). However, such trade-off may be obscured by variation in resource acquisition among genotypes (van Noordwijk \& de Jong, 1986). We tested for the existence of a trade-off between growth and asexual reproduction in two ways. First, we measured the changes in MGR and spore yield over evolutionary time. Here, 
a trade-off will be visible if improvements in one trait will reduce another trait (Novak et al., 2006). Although we see a reduction in asexual spore yield, we do not see a consistent increase in MGR. In our study, the mean MGR of the populations showed a modest increase of $4 \%$ during the first ten weeks of adaptation. MGR can be used as a surrogate measure for fitness for filamentous fungi (Hoekstra et al., 1997; Pringle \& Taylor, 2002; Schoustra et al., 2005). The absence of improvement in MGR in our study indicates that MGR might not be a strong target for selection in our experiment, perhaps because the strains were close to their fitness optima (De Visser et al., 1999; Schoustra et al., 2005). Alternately, MGR based on average growth rate during 5 days may not an accurate measure of fitness in this experimental set up, since after germination of the spores there is very little time for mycelial growth (max 2 days) before the agar surface is completely occupied. It is possible that the initial stages of germ tube formation and outgrowth are more important than the speed of mycelial growth.

The consistent decrease in asexual spore yield was in contrast to our expectations. The design of the experiment involved the serial transfer of asexual spores, and at the start, the inoculum consisted mainly of uninucleate spores. The transfer protocol included a filtration step to exclude mycelial fragments. As the number of spores reduced during transfers, the size of the bottleneck would have also decreased with each transfer. Schoustra et al., (2012) found a negative correlation between mycelial growth rate and the yield of colony-forming units, or asexual spores per surface area, when $A$. nidulans was evolved in the laboratory for 800 generations on a solid medium (Schoustra \& Punzalan, 2012). However, a study using wild isolates of Neurospora crassa evolved in the laboratory on both rich and poor nutrient medium suggests that MGR and spore yield are plastic and largely independent traits and may thus not be correlated (Anderson et al., 2019).

Next, we measured the correlation between growth and reproduction between independently evolved replicate populations at the final time-point. A trade-off here would be visible if the populations took different paths during adaptation to reach the trade-off line (Novak et al., 2006). We did not see a negative correlation between growth and reproduction when we measured the 12 independently evolved populations at week 50 . Instead, we found a marginally-significant positive correlation between the traits (figure 3.2C). When a trade-off between growth rate and yield was tested for the LTEE populations, no evidence was found for a trade-off over evolutionary time or between independently evolved replicate populations. However, the authors did find a negative correlation between growth rate and yield within isolates of the four populations tested, suggesting short-term constraints from a trade-off between these life-history traits (Novak et al., 2006). 
The lack of adaptation we found for MGR and spore yield prompted us to look at competitive fitness to see if adaptation had happened at all. When competitions were carried out between population samples from the final time point and the ancestor, we did not see an improvement in competitive fitness against the ancestor (figure 3.3A). The lack of improvement in fitness against the ancestor prompted us to further analyze two populations in detail by performing pairwise competitions at four-week time intervals. Both the populations tested showed an initial increase followed by a decrease in fitness. These patterns suggested the possibility of non-transitive fitness interactions, where the fitness of an evolved genotype relative to its ancestor is not the sum of the fitness increments relative to its immediate predecessors (Van den Bergh et al., 2018).

Non-transitive fitness interactions can be generated by trade-offs between growth rates in different niches or interference competition, and may especially occur in spatially-structured environments. For example, three bacterial strains, a sensitive strain, a colicin (toxin) producer and a resistant strain, co-existed due to tradeoffs in relative fitness among different pairs of strains in a spatially-structured environment (Chao \& Levin, 1981; Kerr et al., 2002). During the experimental evolution of yeast in a glucose-rich medium both non-transitivity and multilevel selection on the host genome and the intracellular viral genome was observed. The cells (ancestor) that carry the functional viral genome produces a toxin that can kill cells that doesn't carry the viral genome. As populations evolved, adaptive mutations occurred both in the nuclear and viral genome. These adaptive mutations resulted in loss of immunity against the toxin and the ability to produce them. When these evolved populations were competed against the ancestor with a functional toxin producing viral genome, they were killed by the toxin resulting in non-transitive fitness interaction. As populations evolved they experienced multilevel selection on the host genome and the intracellular viral genome it carried (Buskirk et al., 2020).

Non-transitivity occurs when populations are polymorphic due to frequencydependent interactions between contemporary genotypes. Indeed, we found at least two different morphs, one resembling the ancestor ( $\mathrm{AL}$ ), the other having a fluffy appearance (FL), in all populations from week ten onward. The rapid invasion of $\mathrm{FL}$ in the population on $\mathrm{N} 1$ until week 20 (figure 3.4) corresponds to the decline in fitness against the ancestor seen by week 28 (figure 3.3B dashed line). In competitions of population samples from different time points against marked clones from the same populations, the summed fitness increments for population N1 were lower than the values obtained for competition directly with the ancestor. With the hindsight of polymorphisms in the populations, the spore 
color mutant isolated for the experiments has likely originated from the AL morph. The fitness estimates measured across 4-week intervals have not captured the complete population-level fitness increases but perhaps fitness increases of the AL type compared to the populations.

Our study with a multicellular fungus $A$. nidulans did not show improvements in the conventional fitness components MGR and spore yield. Although we saw a divergence in the fitness trajectories for $M G R$, we saw convergence in the reduction in asexual spore yield. At the same time, similar polymorphisms evolved in all populations. FL morphotypes are not uncommon in filamentous fungi. Previous studies with $A$. niger and $A$. oryzae, when grown in glucose-limited chemostats, have given rise to fluffy morphotypes (Van De Vondervoort et al., 2004; Withers et al., 1994). The fluffy phenotype we see might be different than those found in most of the other studies. FL types from all the populations produce abundant aerial mycelium, giving it a fluffy appearance. It also makes spores and is not completely devoid of sporulation (aconidial). Aconidial genotypes following serial sub-culturing through propagule(spores and mycelial fragments) transfer have been reported in A. fumigatus and Neurospora crassa (Bastiaans et al., 2016; Zhang et al., 2015). This morphology in $A$. nidulans can occur due to defects in one or more of the 36 genes involved in light sensing and conidiation (Riquelme et al., 2018). Loss or reduction of conidiation, therefore, seems a common theme among filamentous fungi evolving in laboratory culture conditions.

This study gives us a glimpse at how evolution, in a simple structured environment, gave rise to polymorphisms and complex adaptive dynamics within populations, leading to non-transitive fitness interactions. It opens up many questions. For example, what ecological strategies are used by AL and FL morphotypes for adaptation and co-existence? What mutations, in which genes, give rise to this phenotype? Do the morphotypes show parallel mutations, or do different mutations converge to produce a single phenotype. Do the morphs share a deep genetic divergence from an early time-point, or are we looking at a snapshot of transient dynamics, where these morphs re-evolve repeatedly? These questions are addressed in the next chapters. 


$$
4
$$




\section{CHAPTER 4.}

\section{Test of metabolite interactions underlying experimentally evolved polymorphisms in a filamentous fungus}

Krithi Nandimath ${ }^{1,2}$, Ben Auxier ${ }^{1}$, Alfons J. M. Debets ${ }^{1}$, Sijmen E. Schoustra ${ }^{1}$, Vidyanand Nanjundiah ${ }^{2}$, Duur K. Aanen ${ }^{1}$ and J. Arjan G. M. de Visser ${ }^{1}$

${ }^{1}$ Laboratory of Genetics, Wageningen University, Wageningen, The Netherlands. ${ }^{2}$ Centre for Human Genetics, Bangalore, India 


\section{Abstract}

Twelve replicate populations of Aspergillus nidulans evolved for one year in a structured environment with limiting nutrients. Adaptation to these conditions led to a threefold reduction in reproduction (asexual spore production) in the evolved populations. It also led to the parallel evolution of a polymorphism in seven populations, involving at least two morphotypes: one resembling the ancestor (called ancestor-like, AL) and the other with white cottony aerial hyphae (called fluffy-like, FL). In this study, we test whether metabolic interactions contribute to the maintenance of this polymorphism. We found that contemporary AL and FL types are involved in negative frequency-dependent fitness interactions. By performing fitness assays with media containing metabolic products from the contemporary other morphotype from the final time point, we found no support for a cross-feeding interaction. However, we found that resource competition between the two morphotypes plays a role. 


\section{Introduction}

Understanding the evolution of life's diversity has been a major area of study. Microbes are excellent models to understand how diversity emerges from a monomorphic state and which mechanisms cause the maintenance of this diversity (Elena \& Lenski, 2003). However, according to the competitive exclusion principle (Hardin, 1960), asexual populations evolving in an environment with one limiting resource will be monomorphic, as such an environment will only support one species or genotype. Adaptation in these populations will proceed through selective sweeps of fitter clones in a sequential manner (Muller, 1932). In reality, this simplistic view of clonal replacements is complicated by clonal interference and genetic hitch-hiking (Cvijović et al., 2018). Clonal interference will slow the process of selective sweeps as individuals carrying different beneficial mutations compete with each other and with the ancestor for fixation in the population (Gerrish \& Lenski, 1998). Nevertheless, as long as competition for a single resource drives evolution, polymorphisms are transient states.

Yet, numerous studies have reported the spontaneous evolution of stable maintenance of at least two genotypes in originally clonal populations (Atwood et al., 1951; Bastiaans et al., 2016; Elena \& Lenski, 1997; Frenkel et al., 2015; Helling et al., 1987; Maddamsetti et al., 2015; Morris et al., 2014; Rainey \& Travisano, 1998; Rozen \& Lenski, 2000; P. E. Turner et al., 1996; Zhang et al., 2017). In all cases, negative frequency-dependent fitness interactions among contemporary types are responsible for the stable maintenance of the polymorphism. Negative frequency-dependent interactions occur when a genotype has higher fitness when it is rare than when it is common in a population. Such interactions may arise in homogenous, heterogeneous, structured, and unstructured environments ((Van den Bergh et al., 2018), and references therein), and are caused by fitness trade-offs between adaptations to different "niches" (Rainey et al., 2000). Niches may arise in nonstructured environments from (i) temporal heterogeneity in a single resource (seasonal environment (Vasi et al., 1994)), or (ii) heterogeneity arising from an excreted metabolite (cross-feeding on a metabolizable metabolite). In structured environments, there is more opportunity for niche specialization due to spatial heterogeneities (Rainey \& Travisano, 1998), but negative frequency-dependent coexistence may also arise via toxin- mediated interference competition, for which spatial structure is also essential (Chao \& Levin, 1981; Helling et al., 1987; Rendueles et al., 2015; Wloch-Salamon et al., 2008).

Temporal variation in growth rate and resource abundance is seen in seasonal environments: the serial passage of populations in batch culture during laboratory 
evolution experiments introduces seasonal variation in resource availability and population size. A seasonal environment alone can support diversity in a population due to differential growth rates of the genotypes during the "feast" and "famine" phase (Vasi et al., 1994). In silico experiments have shown that organisms can adapt to a serial-transfer regime by evolving to grow rapidly when resources are abundant or maintain high yields when resources are scarce. These growth strategies can be achieved by gene regulation, niche specialization, and trade-offs between growth and yield either alone or a combination of these strategies (Van Dijk et al., 2019).

Cross-feeding interactions, where partners exchange metabolites that may enhance each other's fitness, is another mechanism of creating multiple ecological niches within an originally homogeneous environment. Many studies have demonstrated that cross-feeding can help maintain diversity in the populations ( $D$ 'Souza et al., 2018; Smith et al., 2019). One of the earliest works that demonstrated the evolution of cross-feeding in an experimental evolution set-up was by Helling et al. (1987). They demonstrated the evolution of acetate cross-feeding in a chemostat culture of Escherichia coli. E. coli may evolve acetate cross-feeding when adapting to growth in a homogenous single-carbon, or a heterogenous multiple-carbon environment (Friesen et al., 2004; Rozen et al., 2009). Cross-feeding has also been demonstrated to sustain diversity in multi-species biofilms (Poltak \& Cooper, 2011).

Spatial structure provides opportunities for niche formation and niche specialization, since it restricts competition among nonmotile genotypes to a local scale and not to a global scale, as seen in homogenous environments, such as applied in most experimental evolution studies. For example, a classical study by Rainey and Travisano (1998) showed the effect of spatial structure on the evolution and coexistence of multiple morphs in Pseudomonas fluorescens elegantly. The structured environment created specific niches with varying oxygen gradients to which the different morphs repeatedly adapted, and competition avoidance drove the maintenance of the morphs (Lind et al., 2015; Rainey \& Travisano, 1998). However, there are reports of stable polymorphism in the absence of structure (Rozen \& Lenski, 2000) and the loss of diversity despite spatial structure (Saxer et al., 2009).

In our previous work (Chapter 3), we described an evolution experiment with 12 populations of the ascomycete fungus Aspergillus nidulans in a structured environment. The experiment was run for one year on a solid synthetic medium with $0.1 \%$ glucose as a sole carbon source with a weekly transfer of $1 \%$ of the asexual spores. We found, surprisingly, that the evolved populations had on 
average not improved their mycelium growth rate or competitive fitness relative to their original ancestor that founded the selection experiment and even had reduced asexual reproductive output. Two populations tested exhibited nontransitive fitness interactions. Non-transitive fitness interactions occurs when the fitness of the evolving genotypes does not increase linearly and occurs when a successor outcompetes its immediate predecessor, but can be outcompeted by a more distant ancestor (Kerr et al., 2002; Van den Bergh et al., 2018). As a possible explanation for these non-transitive fitness interactions, we found that the populations had evolved at least two morphotypes. The morphotypes did not differ in their growth rate and spore yield as monocultures. So, differential growth rate or spore yield did not explain their coexistence. The morphs were present in seven of the twelve populations at the final point.

In this study, we seek to understand the possible mechanism involved in the maintenance of these morphs. We do so by conducting competition experiments between contemporary morphs from the final time point at low and high frequencies to test for frequency-dependent fitness interactions. If the diversity observed in the populations is not transient, we expect to observe negative frequency-dependent interactions between the two morphs. Next, we test for the possible role of metabolites and toxins using conditioned medium (i.e., culture media possibly containing metabolites or toxins produced by one or the other morph growing in the culture medium) for the maintenance of diversity in the populations. When grown on each other's conditioned medium, improvement in growth or spore yield suggests a positive interaction, such as resulting from crossfeeding. A reduction in growth or spore yield when cultivated on the other type's conditioned medium suggests a negative competitive interaction due to resource competition or toxin production.

\section{Materials and methods}

\section{Test of frequency-dependence of fitness interactions}

Before the competitions, the two morphotypes from the final time-point of seven populations which had clear distinct morphotypes were first grown for two weekly transfers on the evolutionary medium to remove the effects of freezing and glycerol in which they are stored. The harvested spores were counted with the Casy TT particle counter (OMNI Life Science \& Co KG, Germany).

After acclimatization, the spores of the 14 morphs were harvested identical to how this was done in the evolution experiment (Chapter 3 ) and counted using the Casy TT particle counter. Spore samples were then mixed in a 1:9 ratio of FL: AL 
and vice-versa. The spores were inoculated on fresh medium at a density of $\sim 10^{6}$ spores per bottle in the evolutionary environment and incubated with loose caps for one week at $37^{\circ} \mathrm{C}$ in the dark. The ratios of both competitors were determined both before and after the competition. The ratios were determined by inoculating counting plates (complete medium with $0.05 \%$ tween $(\mathrm{v} / \mathrm{v})$ ) and counting $50-150$ colonies per competition. With these ratios, the following formula was used to calculate the selection rate constant, $r$, as measure of relative fitness of the $\mathrm{FL}$ morphs (Lenski et al., 1991). Each assay was performed in triplicate.

$$
\begin{array}{r}
r=\ln \left(\frac{\# \text { of evolved colonies after competition }}{\# \text { of ancestral colonies after competition }}\right) \\
-\ln \left(\frac{\# \text { of evolved colonies before competition }}{\# \text { of ancestral colonies before competition }}\right)
\end{array}
$$

\section{Test of metabolic interactions}

Conditioned medium is culture media containing biologically active components that may affect fitness secreted into the media by previously cultured cells. The conditioned medium of each morphotype ( 7 pairs from 7 populations) was prepared by growing each morphotype separately in $100 \mathrm{ml}$ of liquid minimal medium in $500 \mathrm{ml}$ bottles. The bottles were incubated in a $37^{\circ} \mathrm{C}$ incubator at an angle of 45 50 degrees to increase the surface area available for growth for seven days with loose caps. After the incubation period, the mycelial mat that formed on top of the medium was removed and the conditioned medium was filter-sterilized using Steritop, a 0.2-micron bottle top filter from Millipore (cat. No. SCGPS02RE). The sterilized conditioned medium was divided into two aliquots. To one aliquot, $0.1 \%$ glucose and $5 \mathrm{mM}$ urea was added to make a nutrient-supplemented conditioned medium that would reconstitute original glucose and urea levels if both were fully depleted. The other aliquot was used without replenishment. The conditioned medium was used on the same day that it was prepared.

A $10 \%$ water agar solution was prepared freshly and autoclaved on the day of the experiment and kept molten by constant stirring on a heated magnetic stirrer. The Petri plates and bottles to measure growth and spore yield were prepared by diluting one volume of the $10 \%$ agar solution into nine volumes of pre-warmed conditioned medium, and $20 \mathrm{ml}$ was poured into $90 \mathrm{~mm}$ Petri-plates and $10 \mathrm{ml}$ into $30 \mathrm{ml}$ bottles and allowed to cool and solidify on the bench over-night.

Each morphotype was inoculated in four different conditions: on its own conditioned medium with and without nutrient supplementation and on the conditioned medium of its partner with and without supplementation. 


\section{Mycelium density}

To measure mycelial density as a proxy for biomass production, $3 \mu$ l of a spore suspension ( $2 \times 10^{6}$ spores $\left./ \mathrm{ml}\right)$ was spotted in the center of the Petri plates (prepared as mentioned above) and incubated at $37^{\circ} \mathrm{C}$ for three days. The mycelial density was measured by scanning the plates using Epson Perfection V600 Photo flatbed scanner. The images were analyzed in ImageJ. The mycelial density was calculated by eliminating the pixel density values of the medium and the point of inoculation (highest pixel density) and normalized to the size of the colony, resulting in a measure for the average mycelium density.

\section{Spore yield}

To measure spore yield, $50 \mu l$ of the spore suspension ( $2 \times 10^{6}$ spores $\left./ \mathrm{ml}\right)$ was spread on the agar surface in the bottle (prepared as mentioned above) by tilting the bottle and allowing the liquid to spread. The bottles were incubated at $37^{\circ} \mathrm{C}$ for seven days with loose caps to allow the exchange of oxygen. After incubation, the spores were harvested by adding a few (8-10) glass beads and $3 \mathrm{ml}$ of saline tween $(0.005 \%$ tween $\mathrm{v} / \mathrm{v})$ solution and shaking for 10 minutes at $200 \mathrm{rpm}$ on a horizontal shaker. Spores were counted using Casy TT (OMNI Life Science \& Co $\mathrm{KG}$, Germany) following the manufacturer's instruction, resulting in a measure for total spore production.

\section{Results}

\section{Frequency-dependent fitness interactions}

To determine if the stable maintenance of the morphotypes was due to frequencydependent fitness interactions, we performed competition experiments with the two morphotypes from the final time-point of each of the seven populations at low ( 10\%) and high ( $90 \%)$ initial frequency of FL. Relative fitness was measured by the selection rate constant of FL relative to AL. On average, the FL from these seven populations show a significant advantage when rare (Welch two-sample $t$-test, $t=4.24$ and $p=0.0023$ ) and a reduction in that advantage when frequent, supporting a negative frequency-dependent fitness interaction between contemporary morphotypes (figure 4.1). However, when the populations were tested independently, only FL from population N1 had a significant advantage when rare (paired t-test, $p=0.0028$, after correcting for multiple testing using serial Bonferroni correction). Although FL has higher fitness than AL at a frequency of $\sim 10 \%$, it does not have lower fitness than $A L$ at high frequency, hence cannot fully explain their stable coexistence. 


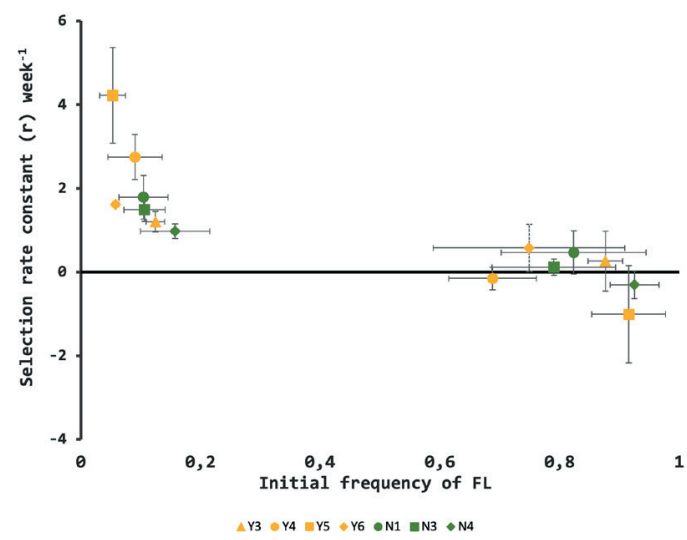

Figure 4.1: Selection rate constant of FL in competition with its contemporary AL from the final time point of seven populations at a frequency of $10 \%$ and $90 \%$. Each data point is the average fitness of three replicate measurements of FL at two frequencies and the error bars are SEM.

\section{Metabolic interactions}

To examine the cause of the negative frequency-dependent interactions at the level of competitive fitness (figure 4.1), we measured two fitness components of both types, reproduction (asexual spore production) and growth (here measured by the increase in mycelial biomass in three days). Although $\mathrm{FL}$ has an advantage when rare in competition with the contemporary AL type (figure 4.1), it does not show significant differences in average growth and spore yield compared to $A L$, when grown independently on fresh medium (t-test, $p>0.05$ for both, figure 4.2).

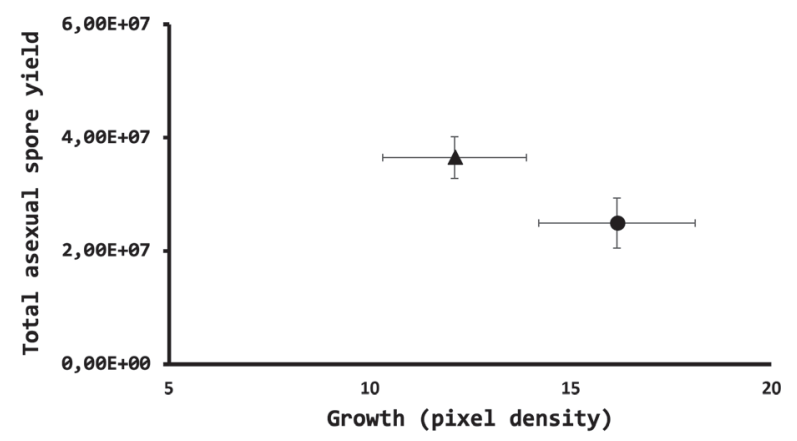

Figure 4.2: Growth and spore yield of FL and AL on fresh medium. Each data point is the grand mean of seven clones of FL (circle) and AL (triangle). Error bars are SEM.

Metabolic interactions like cross-feeding or interference competition via toxin production, could explain the observed negative frequency-dependent fitness interactions (figure 4.1). To test for metabolic interactions, the two morphotypes from the seven populations were grown on their own conditioned medium and that of the other morphotype, both from the final time point in the evolution experiment, and two fitness components, namely growth (biomass production) and spore yield, were 
measured. The results are plotted as effect of the other type's conditioned medium relative its own conditioned medium on each fitness component. The possible outcomes are shown in figure 4.3A. We hypothesized that positive values (higher values on the conditioned medium of the other type than on its own conditioned medium) for either or both components tested would indicate beneficial interactions, such as cross-feeding, and negative values (lower values on the conditioned medium of the other type than on its own conditioned medium) would indicate inhibitory interactions, such as from resource depletion or toxin production.

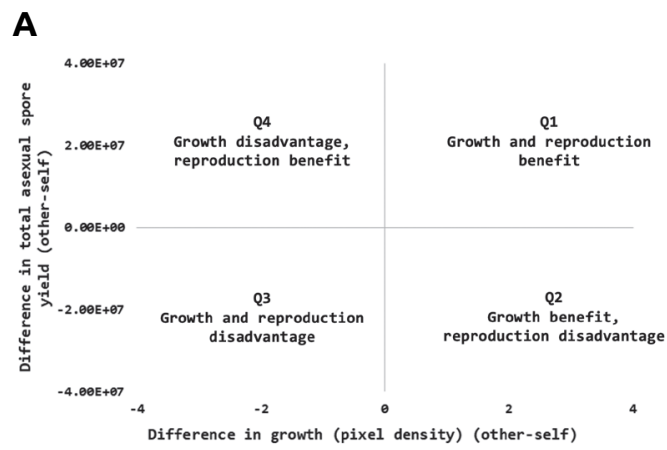

B

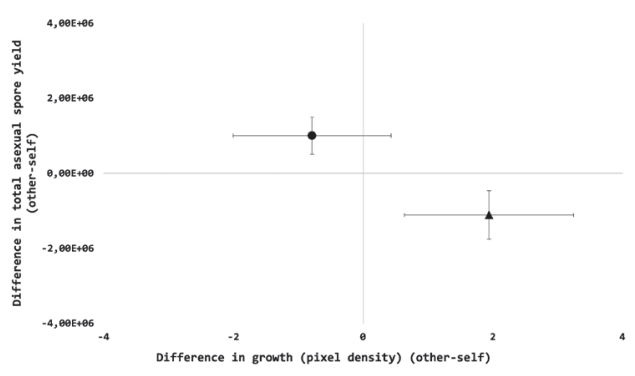

C

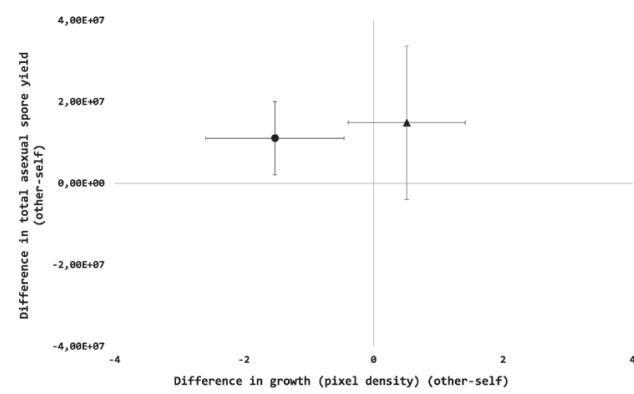

Figure 4.3: Metabolic interactions. (A) Possible outcomes for the conditioned medium results. The results are plotted as the effect of the other type's conditioned medium on each fitness component. Quadrant 1 (Q1) has positive values for both fitness components, which indicates that the fitness components of the morphotype are higher on the partner's (other morphotype) conditioned medium than on its own. Positive values are suggestive that the partner's conditioned medium supports growth and spore production better than the morphotype's own conditioned medium. Quadrant 2 and 4 (Q2 and Q4) has positive values for one fitness component and negative values for the other, indicating that one of the fitness components has higher values and the other has lower values on the partner's conditioned medium. This suggests that the partner's conditioned medium supports one fitness component and inhibits/reduces the other fitness component. Quadrant 3 (Q3) has negative values for both fitness components meaning that the partner's conditioned medium inhibits/reduces both the fitness components of the morphotype compared to its own conditioned medium. (B) Effect of metabolites from the other morphotype on spore production and growth without supplementation. Each data point is grand means of seven clones of FL (circle) and AL (triangle). Error bars are SEM. (C) Effect of metabolites from the other morphotype on spore production and growth with supplementation. Each data point is grand means of seven clones of FL (circle) and AL (triangle). Error bars are SEM. 
The effect of the other type on growth in the absence of supplementation was not significantly different for both types when tested across the seven populations (figure 4.3B, paired t-test, $t=-1.32, p=0.23$ ). However, there is a significant difference between the effect of the other type on spore production (figure 4.3B, paired $t$-test, $t=2.61, p=0.03$ ): $F L$ had a negative effect on the spore production of $A L$, whereas $A L$ has a positive effect on the spore production of $F L$, figure 4.3B. However, this difference in the dependence of reproduction on the presence of the other type's metabolites was not significant when the individual populations are tested separately (paired t-test, $p>0.05$, after correcting for multiple testing with serial Bonferroni correction), indicating that the effects are relatively small compared to the uncertainties in the measurements. Nevertheless, these results suggest that $F L$ types experience a relative reproductive benefit from the presence of contemporary AL types, whereas AL types suffer a detrimental effect, and both types do not significantly affect each other's growth.

The reduction in spore production of $\mathrm{AL}$ on $\mathrm{FL}$ spent medium (figure 4.3B) is indicative of an inhibitory interaction. Such an inhibitory interaction could be either due to the greater depletion of nutrients by FL relative to $A L$ or due to the secretion of a compound that inhibits spore yield of AL. To distinguish between these two hypotheses, we supplemented the spent medium with the original amount of glucose and urea and repeated our assay. If the difference in spore production is due to resource depletion, then supplementation of the conditioned medium should restore the spore yield. If the effect on spore production is due to a toxin, then resource supplementation will not restore spore production as the efficacy of the toxin is not affected by supplementation. The difference in spore production between $\mathrm{FL}$ and $\mathrm{AL}$ is lost upon supplementation of the conditioned medium (figure $4.3 \mathrm{C}$, paired $\mathrm{t}$-test, $\mathrm{t}=-0.30, \mathrm{p}=0.76$ ). The negative effect of $\mathrm{FL}$ on the spore production of AL thus disappeared upon supplementation, while no additional positive effect was seen on FL spore production when grown on supplemented AL-conditioned medium (figure 4.3C, paired $t$-test, $t=1.21$, $p>0.05$ ). Further, when the absolute spore yield on fresh medium (figure 4.2) and supplemented self-conditioned medium are compared, we do not see a difference in the total number of spores produced between the two conditions tested either for $F L$ or $A L$ (Welch two-sample t-test, $t=-0.85$ and $p=0.42$ and $t=-1.38, p=0.20$ for $F L$ and $A L$, respectively). These results therefore strongly suggest that the depletion of nutrients in the FL condition medium is responsible for the lower spore yield of AL. 


\section{Discussion}

In this chapter, we set out to identify the nature of the fitness interactions between two dominant morphotypes repeatedly observed in an evolution experiment with the ascomycete fungus $A$. nidulans. During evolution in a structured environment for one year on minimal nutrients and weekly transfer of $1 \%$ asexual spores to fresh medium, seven of the 12 populations maintained an evolved polymorphism of at least two phenotypically distinct types, one resembling the ancestral phenotype, the other with a fluffy appearance. The evolved populations did not show increased competitive fitness relative to the ancestor, but showed nontransitive fitness interactions, based on detailed analyses of two populations (chapter 3). The polymorphism arose early during evolution in all 12 populations, and was maintained until the end of the experiment in at least seven populations.

To understand the mechanism causing their maintenance in the population, we carried out competitions with the two contemporary morphotypes from the final time point at both low and high initial frequencies. The fitness of one type relative to the other can show a positive, a negative or no dependence on its frequency. From our data, we saw that the FL type has an advantage when it is rare in the population. But unexpectedly, there was no fitness loss at $\sim 90 \%$ frequency, although the fitness advantage was lower than at low frequency; thus, the fitness advantage depends on the frequency (figure 4.1). A similar observation has been reported for the long-term experimental evolution (LTEE) populations of $E$. coli (Lenski et al., 1991). One genotype in the Ara-1 population at 7500 generation shows a reduction in fitness as its frequency increases, but still has higher fitness than the other genotype with which it coexists at all frequencies tested (Maddamsetti et al., 2015). There can be two explanations for this observation: (i) The equilibrium frequency is not within the range of frequencies tested. (ii) The evolution environment changed due to the secretion of metabolites or toxins during the experiment, which is not recapitulated in the competition environment with the two dominant types. For our results, option two might be responsible for the observations. We can exclude option one, as the data from chapter 3 (figure 3.5 ) show that the grand mean frequency of $F L$ is $\sim 50 \%$, well within the range of frequencies we tested (i.e., $10 \%$ and $90 \%$ ).

Next, we tested whether ecological interactions may underlie the observed negative frequency dependence of fitness interactions between $A L$ and FL types. We focused on metabolic interactions and tested for both positive and negative interactions by using media conditioned by the growth of each type in our assays. One can expect three possible scenarios: (i) Both morphotypes differ in their ability 
to deplete the limiting resource, glucose; (ii) One or both morphotypes produce an inhibitory substance; (iii) One or both morphotype cross-feed on an excreted metabolite by the other type. Our results (figure 4.3B) show that when AL is cultured on FL conditioned medium, it produces fewer spores than when cultured on its own conditioned medium. In contrast, FL produces relatively more spores on medium conditioned by AL. The reduction in AL spore yield, when grown on unsupplemented FL-conditioned medium disappears when grown on supplemented FL medium (figure 4.3C). This result, combined with the lack of increase in FL spore production on supplemented $A L$ medium suggests that $F L$ is a superior resource utilizer to AL. The lack of usable nutrients in FL's un-supplemented spent medium was responsible for the lower spore yield of AL. The higher reproduction of FL on AL-conditioned medium could be due to lesser depletion of glucose by AL.

Fluffy mutants have been seen to evolve in the laboratory in other filamentous fungi like Neurospora crassa and Aspergillus fumigatus (Bastiaans et al., 2016; Zhang et al., 2017). In N. crassa, the fluffy-like mutants are deficient in somatic fusion and gain an advantage at a low frequency when there is a high probability of wild-type mycelia fusing with them. Under such conditions, fluffy-like mutants are more likely to be over-represented in spores (Grum-Grzhimaylo et al., 2021). We did not test the ability for somatic fusion of our morphotypes, but we consider it possible that FL and AL types in our experiment also engage in intra-somatic interactions, in addition to resource competition, which may contribute to their stable co-existence. Our findings also show some resemblance with those of Rozen and Lenski (2000) with one of the LTEE populations. They found two stably coexisting morphs engaged in a negatively frequency-dependent interaction. One of the morphs influenced the survival of the other morph by removing essential nutrients in the medium and grew better on the conditioned medium than on the fresh medium (Rozen et al., 2009).

In this study, we have shown that when adapting to a simple homogeneous laboratory environment, fungi can give rise to phenotypic diversity. Negative frequency-dependent competitive interactions seem to maintain this diversity. There are metabolic interactions between the two morphotypes FL and AL; FL reduces the spore yield of the $A L$ by reducing the available nutrients for $A L$, e.g., being more efficient at utilizing nutrients. Physical interaction between FL and AL types may also be a possibility. For example., FL type may produce more aerial hyphae that may overgrow and hinder sporulation of AL type. 



\section{CHAPTER 5.}

\section{Genomic basis of a repeatedly evolved polymorphism in experimental populations of Aspergillus nidulans}

Krithi Nandimath 1,2, Joost van den Heuvel ${ }^{1}$, Ben Auxier ${ }^{1}$, Eric Bastiaans ${ }^{1}$,

Duur K. Aanen ${ }^{1}$, and J. Arjan G. M. de Visser ${ }^{1}$

${ }^{1}$ Laboratory of Genetics, Wageningen University, Wageningen, The Netherlands. ${ }^{2}$ Centre for Human Genetics, Bangalore, India 


\section{Abstract}

Twelve replicate populations initiated with a laboratory strain of the ascomycete fungus Aspergillus nidulans evolved on synthetic minimal glucose agar medium for one year, using weekly transfers of $1 \%$ of the produced asexual spores to fresh medium. This Aspergillus short-term evolution experiment (ASEX) was designed to understand how filamentous fungi adapt to growth on limited carbon in a spatially structured environment. As noted in the previous chapters, we observed no systematic improvement in the fitness components tested and neither in the competitive fitness relative to the ancestor. Instead, we observed the repeated evolution of at least two morphotypes, with a fluffy-like (FL) or an ancestor-like (AL) colony morphology, leading to non-transitive fitness interactions among isolates in two selected populations. The genomic analyses of clones from all 12 populations at an early (week 10) and the final time point (week 52), which we present here, show a clear role of natural selection during ASEX. We also observed a shared genetic basis and different timing of adaptation of AL and FL types. In addition, in most populations both morphotypes do not form monophyletic groups, but they frequently disappear and re-evolve from ancestral forms of both types. Reduction in asexual spore yield, the most evident parallel phenotypic change found in all our evolved populations, is not due to direct selection on genes involved in asexual reproduction. Instead, we argue that reduced spore yield is a pleiotropic effect of adaptive changes in metabolism. 


\section{Introduction}

Natural selection of a population adapting to a novel environment occurs by differential survival and reproduction. Adaptive mutations can fine-tune existing functions by altering gene expression, regulatory networks and metabolic fluxes. The early, large effect mutations that are seen in laboratory evolution experiments with microbes often occur in regulatory genes, such as those encoding RNA polymerase core enzymes like RpoC, where mutations were found in replicate populations of Escherichia coli adapting on minimal medium (Conrad et al., 2010). Mutants of rpoC grew faster and converted carbon to biomass more efficiently on minimal medium than on rich medium (Conrad et al., 2010). Thus, an important class of adaptive mutations in laboratory evolution experiments alters transcription kinetics and levels (Conrad et al., 2011; Philippe et al., 2007). Adaptation can also occur by mutations unlocking novel functions, such as enzymes with increased catalytic activity on specific substrates. Such gain of function mutations may enable organisms to occupy new niches. Along with genetic mechanisms, ecological opportunity is another important driver for evolution by creating conditions for the spread of a novel function (Barrick \& Lenski, 2013; Kassen, 2019). For example, the addition of citrate to the growth medium provided the opportunity for the evolution of citrate utilization in one of the Escherichia coli long-term evolution experiment (LTEE ) populations (Blount et al., 2008, 2012; Lenski et al., 1991). Another classical example is the evolution of three morphotypes of Pseudomonas fluorescens in a spatially structured environment, where the structure created distinct niches with varying oxygen availability and other competitors to which the different morphs repeatedly adapted (Lind et al., 2015; Rainey \& Travisano, 1998).

In a spatially structured environment, the kinds of adaptive mutations are likely to be different than those in a well-mixed environment. For example, populations of phage $\lambda$ growing in spatially structured environments with host bacteria without mixing, were selected for reduced virulence relative to populations growing in well mixed environments (Berngruber et al., 2015). This was due to reduced scope for horizontal transmission of the phage, favoring more prudent growth strategies. The benefits of faster growth may be lower as spatial structure limits competition among evolving genotypes to local scales. Furthermore, the rate of adaptation in a structured environment is slower due to inefficient local resource competition (Habets et al., 2006, 2007; Perfeito et al., 2008). Potentially, improvements in other fitness components like increased dispersal or interference competition may have increased relevance (Chao \& Levin, 1981; Wloch-Salamon et al., 2008). 
With the combined forces of experimental evolution and low-cost whole genome sequencing, evolutionary biologists can address the genomic basis of adaptation, and the relative roles of selection and drift and the repeatability of evolution at new levels of resolution (Barrick \& Lenski, 2013). Parallel evolution, the repeated evolution of the same genotype or phenotype can be quantified using the Jaccard Index $(\mathrm{J})$, i.e., the ratio of the number of mutated genes shared by at least two populations to the total number of genes mutated in both populations, which ranges from 0 (no shared mutations) to 1 (all mutations shared). A meta-analysis of nine bacterial and seven yeast laboratory evolution studies has found that most $J$ values are close to 0 , but some are greater than 0.5 , showing that there is a large variation in the degree of parallel evolution (Bailey et al., 2017). A spatially heterogeneous environment with patchy resource distribution generates differential selection pressures between patches. These conditions, combined with varying dispersibility, will result in the generation of either specialists or generalists (Bailey et al., 2015). The degree of parallel evolution depends on the combination of environmental heterogeneity and amount of dispersal of the populations (Bailey et al., 2015).

The Aspergillus short-term evolution experiment (ASEX) was designed to understand how filamentous fungi adapt to growth on limited carbon in a spatially structured environment. Twelve replicate populations initiated with a laboratory strain of Aspergillus nidulans evolved on synthetic minimal glucose agar medium for one year, using weekly transfers of $1 \%$ of the produced asexual spores to fresh medium. One of the striking observations was the reduction in asexual sporulation across all 12 populations. Apart from reduced sporulation (chapter 3 ), the evolved populations showed the following distinct characteristics: (i) no improvement in mycelium growth rate and competitive fitness compared to the ancestor (chapter 3), (ii) non-transitive fitness interactions among isolates from different time points in two selected populations (chapter 3 ), and (iii) multiple populations showed the presence of at least two colony morphotypes, with a fluffy-like (FL) and an ancestorlike (AL) colony morphology from week 10 onward (chapter 3). These morphotypes showed an overall tendency towards weak negative frequency-dependent fitness interactions and resource competition (chapter 4).

In this chapter, we explore the role of natural selection, the genetic basis of the evolved polymorphisms and the mechanisms of adaptation of the ASEX populations, by sequencing clones from both morphotypes from all 12 populations at two time points of the evolution experiment. 


\section{Materials and methods}

\section{Evolution experiment and morphotypes}

We started six replicate populations each of $A$. nidulans strains WG649 and WG650, which are isogenic except for differences in nitrate non-utilizing mutations and spore color. WG649 has wild-type green spores and a nia mutation and WG650 has yellow spores and a $c n x$ mutation; both mutations cause autotrophy for using nitrate as a nitrogen source. The six WG649 populations are henceforth referred as N1-N6 and six WG650 populations as Y1-Y6. The strains were inoculated in $30 \mathrm{ml}$ glass bottles with $10 \mathrm{ml}$ of solid minimal media (MM) (Pontecorvo, 1953) supplemented with $0.1 \%$ glucose and $5 \mathrm{mM}$ urea. $1 \%$ of the asexual spores were transferred weekly to fresh medium for a year. We found two morphotypes, named fluffy-like and ancestor-like, from as early as week 10 and until the end of the experiment in seven of the 12 populations. The term fluffy-like (FL) refers to colonies with a white cottony appearance, while the term ancestor-like (AL) refers to colonies resembling the ancestral colony phenotype with abundant conidiospores. For all populations at two time points, week 10 and week 52 (and week 20 in the case of Y6), one clone of each morphotype, or in those cases where only one morphotype was present, a single type, was sequenced.

\section{DNA extraction}

The stains were grown in liquid minimal medium with $1 \%$ glucose and $5 \mathrm{mM}$ urea and shaken at $100 \mathrm{rpm}, 37^{\circ} \mathrm{C}$ for 48 hours. A. nidulans grows as small pellets of mycelium in shaken liquid medium. After 48 hours, the mycelium was separated from the liquid by filtering in through a Büchner funnel with a Whatmann filter paper attached to a vacuum pump. The DNA was extracted using a modified protocol from Rogers S.O (1994). Briefly the dried mycelium was finely ground using liquid nitrogen in a mortar and pestle. The ground contents were transferred into a tube and $4 \mathrm{ml}$ of hot $2 \%$ CTAB was added and shaken to obtain a homogenous, viscous suspension. This suspension was incubated at $65^{\circ} \mathrm{C}$ for 1 hour. This was followed by the addition of equal volume of chloroform: isoamyl alcohol (24:1) and rotated at room temperature (RT) for 20 minutes followed by centrifuging for 30 minutes. The upper phase was transferred and 0.1 volume of hot $10 \%$ CTAB added and mixed gently. Then equal volume of chloroform: isoamyl alcohol (24:1) was added and extracted at RT for 20 minutes by rotation and centrifuged at RT for 30 minutes. The upper phase was separated and equal volume of $1 \%$ CTAB added. The DNA was precipitated by inverting the tube gently several times (precipitation should be visible). The DNA was pelleted by centrifuging at RT. The pellet was resuspended in hot high salt TE and 2.5 volumes of ice-cold $96 \%$ ethanol added and mixed carefully. The DNA was then pelleted and washed in $70 \%$ cold ethanol 
and centrifuged. The DNA pellet was dried in a vacuum exicator and dissolved in $0.1 \times \mathrm{TE}$.

\section{Reference-based mapping and variant calling}

A pipeline similar to (Engel et al., 2020; Grum-Grzhimaylo et al., 2021) was used, with slight modifications. Raw FASTQ files were trimmed and filtered by TRIMMOMATIC ( $v$ 0.27, LEADING:3, TRAILING:3, SLIDINGWINDOW:4:15, MINLENGTH:70, (Bolger et al., 2014)). The resulting trimmed reads were aligned using bwa mem ( $v$ 0-715, default parameters, Li 2013) using the Aspergillus nidulans FGSC A4 genome assembly (GFC_000149205.2_ASM14920v2_genomic.fna). Reads with mapping quality lower than 20 were filtered using samtools (v 0.1.19, (Li \& Durbin, 2009)). We then removed duplicates using picard tools (v.1.109, MarkDuplicates, http:// picard.sourceforge.net) and performed realignment around indels with GATK ( $v$ .3.7-0, (McKenna et al., 2010)).

The resulting BAM files were combined in mpileup format (samtools, mpileup, default parameters, v 0.1.19, (Li \& Durbin, 2009)) after which SNPs and indels were separately called using varscan ( $v$ 2.3.9, using mpileup2snps and mpileup2indel resp., --output-vcf 1, --min-var-freq 0.05 (Koboldt et al., 2009)) for which we only kept those variants that differed between the samples (i.e, not between all the samples and the reference) and was subsequently annotated using SNPeff (v4.3, (Cingolani et al., 2012)) using GCF_000149205.2_ASM14920v2_genomic.gff as gene model input. The resulting vcf files were inspected for genetic differences between any pairwise comparison between the ancestor and any evolved strain, using vcfR (v 1.8.0, (Knaus \& Grünwald, 2017)), for which we used a minimum allele frequency difference of 0.9 and a minimum coverage of 10 to filter SNPs and indels. Every resulting variant was then visually inspected in all samples using IGV (v 2.4.14, (Robinson et al., 2011)).

For larger structural variation (including large indels) we used unfiltered BAM files and filtered out all reads with soft and hard clipped reads, as well as indel and deletion calls. Furthermore, we filtered on flags $67,131,115,179,81,161,97$, $145,65,129,113$ and 177 which potentially indicate large indels or deletions or chromosomal rearrangements. We then quantified coverage of all these reads in 100 bp windows using TIDDIT (v 2.2.6 (Eisfeldt et al., 2017)). The resulting coverage distribution was divided to the total coverage of the initial BAM files which therefore yielded the frequencies of 'alternate call' mapped reads to those with 'normal' mapping. These frequencies were then compared between all pairwise samples, ordered upon frequency and visually inspected using IGV without any a priori cutoffs. 


\section{Expected number of genes with parallel mutations}

To predict the number of genes that would be expected to be mutated in parallel in the absence of natural selection, from a sample size equal to our observed data, we simulated 104 random mutations to the Aspergillus genome. The genes affected were randomly selected, weighted according to length, and 1000 permutations were performed.

\section{Strength of selection of parallel and private mutations}

To determine the strength of selection, we first calculated the expected ratio of non-synonymous to synonymous mutations $(\mathrm{N} / \mathrm{S})$ while considering mutational bias. Based on our empirical mutation spectrum, with transitions being twice as likely as transversions, we performed 10,000 simulated mutations, and recovered an expected N/S ratio of 2.82. Then, we calculated the observed N/S ratio for three sets: (i) all mutations, (ii) mutations in genes not found in parallel, and (iii) mutations in genes found in parallel. This observed $\mathrm{N} / \mathrm{S}$ ratio was then divided by the expected $\mathrm{N} / \mathrm{S}$ ratio to give the $\mathrm{dN} / \mathrm{dS}$. Note that this calculation has a different basis than the $\mathrm{dN} / \mathrm{dS}$ commonly used in population genetics.

\section{Test for sexual crossing}

Plates $\left(90 \mathrm{~mm}\right.$ ) with minimal crossing agar media (For $1000 \mathrm{ml}$, add $1.0 \mathrm{~g}$ of $\mathrm{NaNO}_{3}$, $1.5 \mathrm{~g}$ of $\mathrm{KH}_{2} \mathrm{PO}_{4^{\prime}} 0.5 \mathrm{~g}$ of $\mathrm{MgSO}_{4} \cdot 7 \mathrm{H}_{2} \mathrm{O}, 0.5 \mathrm{~g}$ of $\mathrm{KCl}, 20 \mathrm{~g}$ of glucose, $1 \mathrm{mg}$ of FeSO $4^{\prime}$ $\mathrm{ZnSO}_{4}, \mathrm{MnCl}_{2}$ and $\mathrm{CuSO}_{4}$. Adjust pH to 5.8 with $1 \mathrm{M} \mathrm{NaOH}$ ) supplemented with urea $(5 \mathrm{mM})$ were inoculated in the middle with a $5 \mu \mathrm{l}$ droplet of conidial suspension containing abundant conidia $\left(\sim 10^{6}-10^{7}\right.$ conidia/ml). Three replicate plates were prepared for each tested isolate. Plates were incubated for three days at $37^{\circ} \mathrm{C}$, after which the plates were sealed with a double layer of parafilm. Plates were then incubated again at $37^{\circ}$ for another 18 days before analysis. To determine whether the isolates could successfully form fruiting bodies, a qualitative visual check was performed. This was done under a dissecting microscope with 10x-50x magnification and a micro lancet dissection needle to remove overgrowing mycelium and conidia.

\section{Test for mycelial fusion}

To test whether the soft homologue is involved in fusion we selected evolved types that had a mutation in this gene. Two of the selected types had the $c n x$ marker and two had the nia marker. The isolates were tested in a complementation test setup for which fusion is required. Conidia of a nia isolate were co-inoculated with conidia of a $c n x$ isolate by putting a 5 ul droplet of mixed conidia suspension (containing $2 \times 10^{6}$ conidia $/ \mathrm{ml}$ ) on a plate with minimal medium with nitrate as a source of nitrogen. As a control the isolates were also inoculated individually 
on minimal medium to confirm the predicted (lack of) growth. The plates were incubated at $37^{\circ} \mathrm{C}$.

\section{Results}

\section{Summary of mutations}

In total, we sequenced 44 clones (evolved morphs) from all 12 populations at two-time points of the evolution experiment, week 10 (early) and week 52 (final). The 44 evolved clones were categorized based on their colony phenotype into ancestor-like $(A L)$, fluffy-like $(F L)$ and undetermined $(U)$ type. We sequenced 22 early time-point clones (10 AL, $10 \mathrm{FL}, 2 \mathrm{U}$ ) and 20 final time-point clones ( $8 \mathrm{AL}, 8$ $\mathrm{FL}, 4 \mathrm{U})$. We found a transition to transversion ratio of 1.08 across our populations. Synonymous mutations and premature stop codons made up $20 \%$ each of the observed SNP's and missense mutations contributed 59\%, while $12 \%$ were indels. We found a single $5 \mathrm{~kb}$ insertion in an intergenic region and the rest of the indels were $<3-4$ base pair changes (figure 5.1). The details of the distribution of the mutations at week 52 among the different populations and their predicted function is given in table 5.1 .

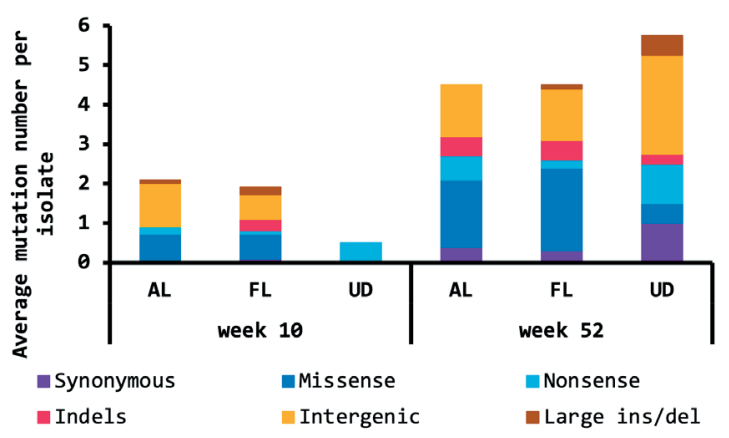

Figure 5.1: Frequency of mutation types per morphotype at two time-points. The non-synonymous mutations are divided into missense and nonsense types. Indels are $<4$ bp changes and large ins/del are $>1 \mathrm{kbp}$ insertions and deletions. The values are number of mutations of each type averaged per morphotype. $A L=$ ancestor-like $(n=10$ at week 10 and 8 at week 52$), F L=$ fluffy-like $(n=10$ at week 10 and 8 at week 52) and UD $=$ undetermined ( $n=2$ at week 10 and 4 at week 52).

\section{Parallelism and positive selection}

Next, we tested the role of natural selection in our experiment by comparing the rates of nonsynonymous to synonymous substitutions in all the sequenced clones from both time points. We found a total of 43 non-synonymous and 11 synonymous substitutions when combining the results of both time-points, resulting in an N/S ratio of 3.91. The expected $\mathrm{N} / \mathrm{S}$ ratio is 2.82 after correcting for the potential number of nonsynonymous and synonymous mutations and transition/transversion bias in the absence of selection, resulting in a genome-wide $\mathrm{dN} / \mathrm{dS}$ value of 1.38. 
This observed $\mathrm{dN} / \mathrm{dS}$ is not significantly different from the expected $\mathrm{dN} / \mathrm{dS}$ of 1 in the absence of selection using the Clopper-Pearson binomial test $(p=0.361)$. We split the non-synonymous and synonymous substitutions for the two time-points: at week 10 we found 15 non-synonymous and 3 synonymous substitutions and at week 52, 30 non-synonymous and 8 synonymous substitutions. The $\mathrm{dN} / \mathrm{dS}$ for the two time-points are 1.77 and 1.32 respectively. These are not significantly different from the genome-wide $\mathrm{dN} / \mathrm{dS}$ value.

We then looked separately at the $\mathrm{dN} / \mathrm{dS}$ ratio for genes that had been hit by mutations in only a single population versus genes hit by mutations in multiple populations. For the first class, we found 15 non-synonymous and 10 synonymous mutations resulting in an average $\mathrm{dN} / \mathrm{dS}$ of 0.46 , which is not significantly different from $d N / d S=1$ in the absence of selection ( $p=0.178$, figure $5.2 A$ ). However, looking at genes that were mutated in more than one population, we found 29 nonsynonymous and 1 synonymous mutation resulting in a dN/dS value of 10.28 , which was significantly different from neutral expectations ( $p=0.003$, figure $5.2 \mathrm{~A}$ ). In addition, we simulated the probability of multiple genes being affected by 104 (sample size equal to our observed total mutation number) randomly placed mutations across all samples (figure 5.2B). This revealed that the eight genes with parallel mutations in our experimental data was higher than found in simulations, where 1,000 independent runs never had more than five genes with parallel mutations. Therefore, we conclude that the genes which were mutated twice or more in our evolution populations, are likely under positive selection.

A)

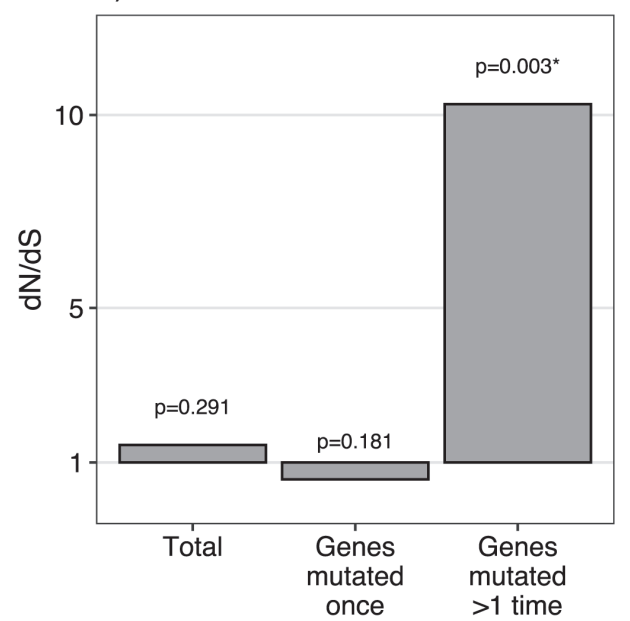

B)

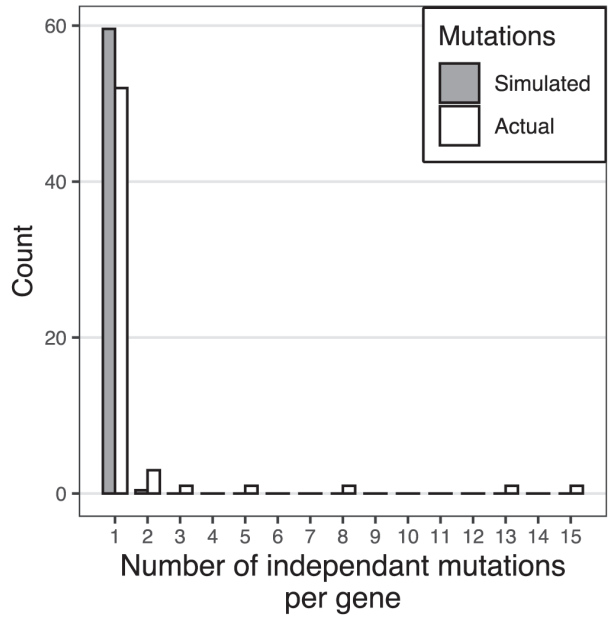

Figure 5.2: Test of the role of natural selection. (A) $\mathrm{dN} / \mathrm{dS}$ ratios inferred for all genes, genes with a mutation in a single genotype and genes with mutations in multiple genotypes across the entire dataset. (B) Probability of parallel mutations in actual data vs simulated data under the neutral assumption that they occur randomly. 


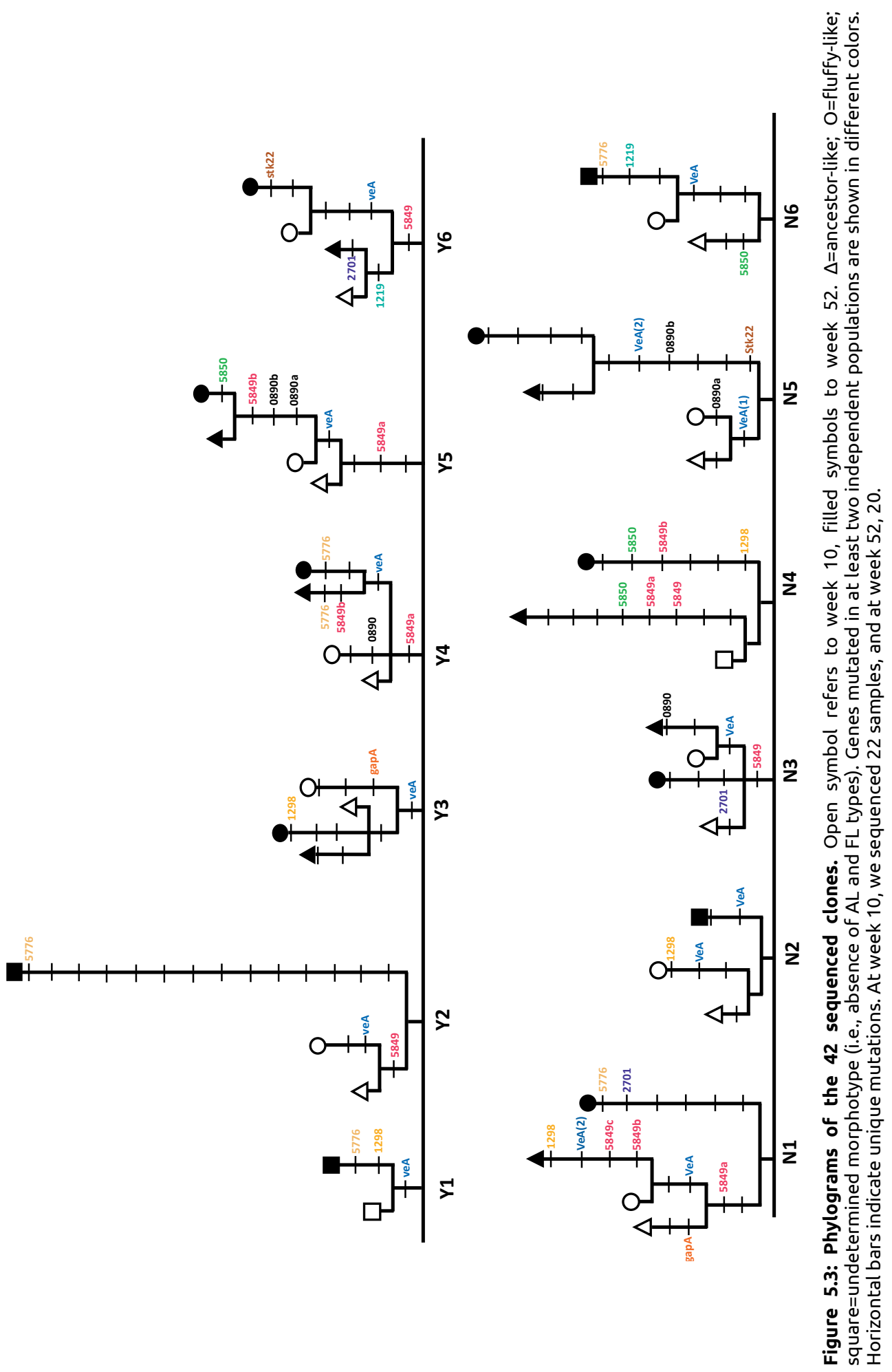




\section{Phylogenies of the morphotypes}

To examine the evolutionary history of the morphs and to understand whether the two types share a common genetic basis, we constructed phylograms for the sequenced clones in which we highlight genes showing parallel substitutions (figure 5.3). Both morphs were at least present at one time point in 11 of the 12 populations, with one population (N4) where they appear late and three populations (Y2, N2 and N6) where they were present at week 10 and have been lost by week 52 . Of the remaining six populations, the early and late morphs are monophyletic only in population (Y6), while in the other five either one or both morphs at week 52 derive from the ancestor or other morphotype, indicating that these morphotypes repeatedly evolve from different genetic backgrounds.

\section{Parallelism and phenotype}

From the phylogenies we can see that the morphotypes do not always share deep divergence. To address whether the morphotypes have a shared genetic basis, we first calculated the fraction of mutations shared among pairs of genotypes with similar and different morphotype. Figure 5.4 shows the average fraction of mutations shared within and between morphotypes at each time point. The FL morphs from different populations at week 10 share on average $35 \%$ of their mutations, while the AL morphs share only $21 \%$ of the mutations, while between $\mathrm{FL}$ and $\mathrm{AL}$ morphs only $18 \%$ of the mutations are shared. At this early time point, the fraction of mutations shared among $\mathrm{FL}$ morphs is significantly higher than among $A L$ morphs and between $F L$ and $A L$ morph (paired t-test, $p<0.001$ ), indicating a partially shared genetic basis for the FL morphotype. The fraction of mutations shared by AL morphs is not significantly higher than the fraction they share with FL types $(p=0.11)$, suggesting no specific common basis for AL initially. Remarkably, after a year (week 52) the fraction of mutations shared between FL morphs has dropped to $18 \%$ for and is significantly lower relative to week 10 $(p<0.001)$. However, we see an increase in the fraction of shared mutations among the AL morphs to $33 \%$ and a significant increase relative to week $10(p<0.01)$ and relative to mutations shared between $\mathrm{AL}$ and $\mathrm{FL}(25 \%)$. The fraction of mutations shared by $A L$ morphs is then even higher than that within FL types and between $F L$ and $A L(p<0.001)$. However, the fraction of mutations shared by $A L$ and $F L$ and $A L$ combinations is not significantly different $(p=0.18)$. These results suggest early parallel adaptation followed by divergence of FL morphs and a later parallel adaptation in AL morphs possibly in response to the FL morphs. 


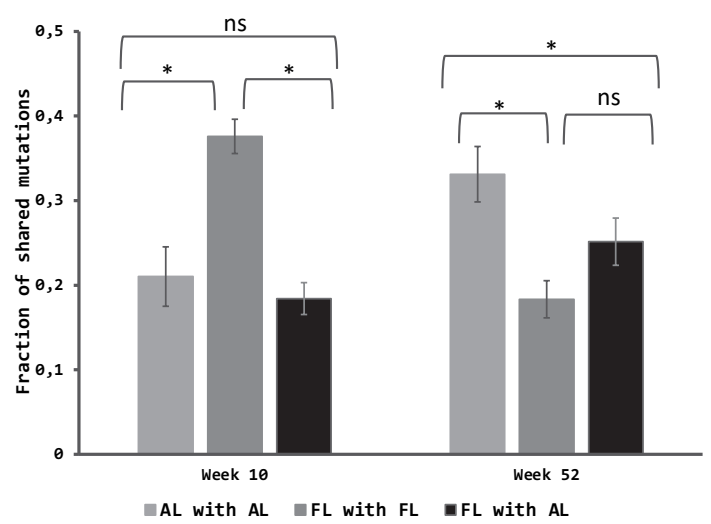

Figure 5.4: Fraction of shared mutations within and between AL and FL types. Each bar represents the average value across the populations and the error bars are SEM. * $=$ significant $(p<0.05$, paired t-test), ns = non-significant.

\section{Genes under positive selection}

We found eight genes that were mutated independently in at least two evolved populations. Those are listed in table 5.1 with their predicted functions. None of these mutation targets uniquely defines either of the two morphs, rather they contribute to the evolution of AL and FL morphs in quantitatively different ways. The early evolution of FL morphs at week 10 is particularly due to parallel mutations in veA (Fisher's exact $\mathrm{p}=0.0089$ ). As the evolution experiment progressed, the $\mathrm{AL}$ morphs evolved to share also mutations in veA, AN5849 and mutations in both these genes are observed in a larger fraction of the morphotypes (figure 5.5).

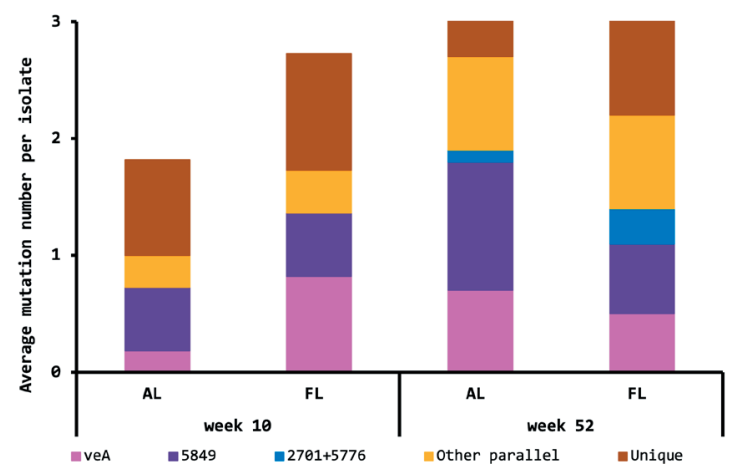

Figure 5.5: Mutation frequencies in AL and FL type in genes affected in multiple clones (parallel) versus in genes affected in a single clone (unique). Each block represents the average frequency of mutations in a particular gene per morphotype. $A L$ = ancestor-like, $\mathrm{FL}=$ fluffy-like. 


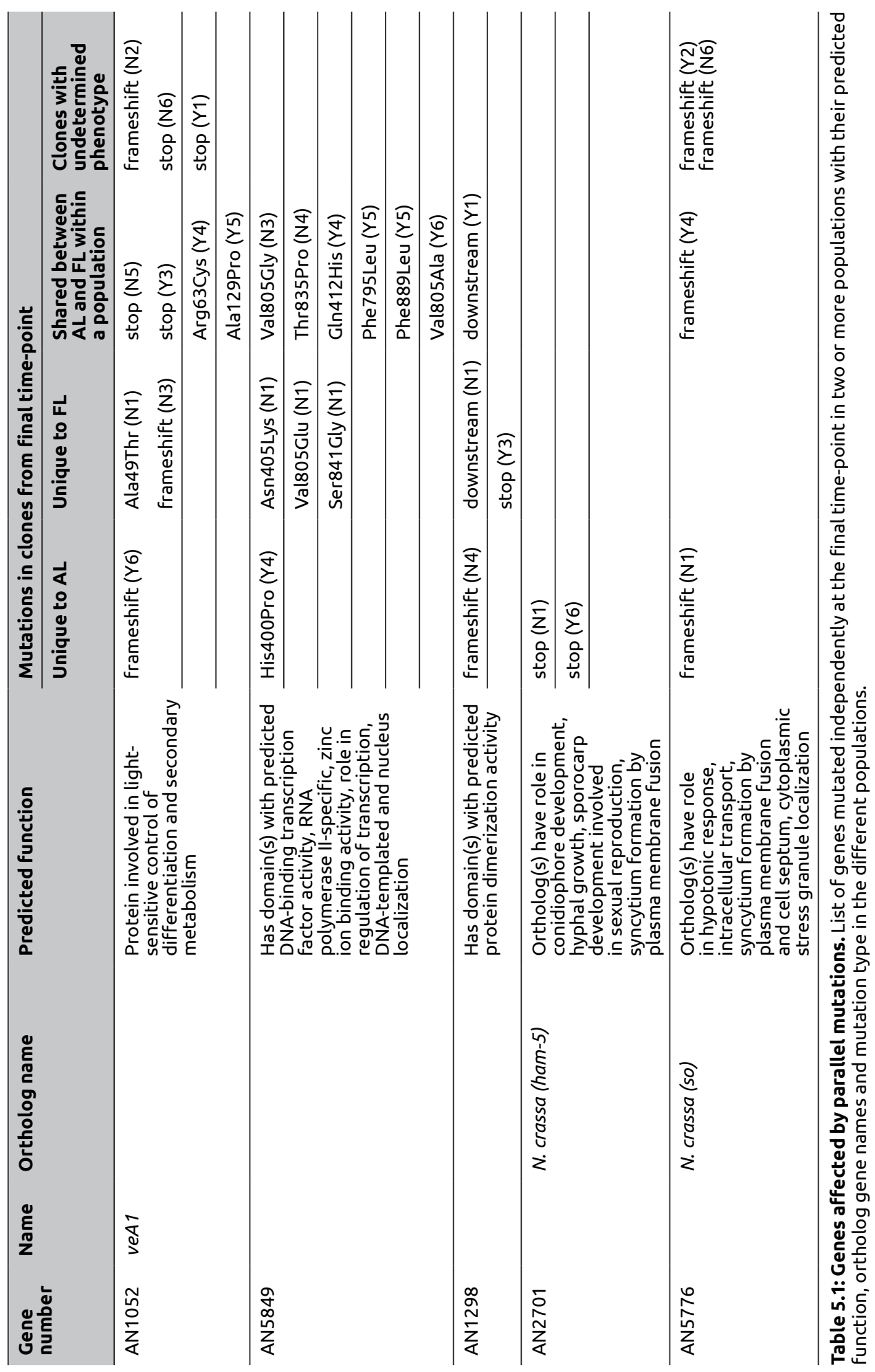




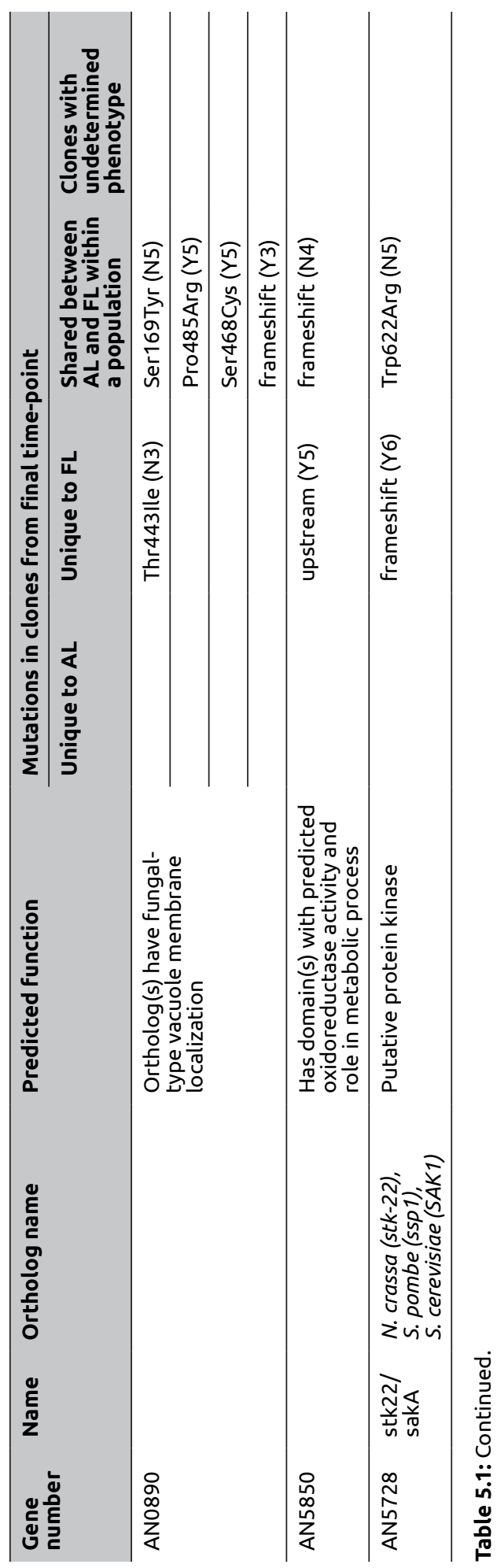




\section{Role of common mutations in asexual sporulation, metabolic interaction, sexual reproduction and hyphal fusion}

\section{Asexual sporulation}

The most visible adaptation we observed was the reduction in sporulation of the evolved populations. Although the populations had reduced asexual spore production, we did not see significant differences in spore yield between the $\mathrm{AL}$ and $\mathrm{FL}$ morphs isolated (Chapter 3). In the earlier observations, the isolated clones were grouped based on differences in their colony morphology phenotype. We now grouped the isolates based on their genotypes, to see the effect of the genotype on spore yield. For this, we grouped the early time-point clones into four categories, namely mutants of veA (Y1, Y2FL, Y5FL, N2FL, N5FL, N5AL and N6FL), mutants of AN5849 (Y2AL, Y4FL, Y4AL, Y6AL, N1AL, N3AL), double mutants of veA and $A N 5849$ (Y6FL, N1FL, N3FL), and mutants having unique mutations ( $\mathrm{Y} 3 \mathrm{AL}$, $\mathrm{N} 2 A L, N 4, N 6 A L)$. The comparisons between mutant and wild-type alleles at the two loci did not yield any significant differences in spore yield between groups (see figure 5.6A).

In Neurospora crassa, mutations in the genes soft and ham-5 are known to cause a reduction in asexual spore yield (Bastiaans et al., 2016; Grum-Grzhimaylo et al., 2021). We tested the role of mutations in orthologs of ham-5 and soft (AN2701 and AN5776, respectively) in asexual spore production in our $A$. nidulans isolates. For this, we grouped the final time-point clones into two groups, one with clones which had mutations in ham-5 and/or soft (Y1, Y2, Y4FL, Y4AL, N1FL and N6) and the second group with all other mutations without mutations in ham-5 and soft. However, this comparison did not yield a significant difference in average spore yield between the groups either (figure 5.6B).

A

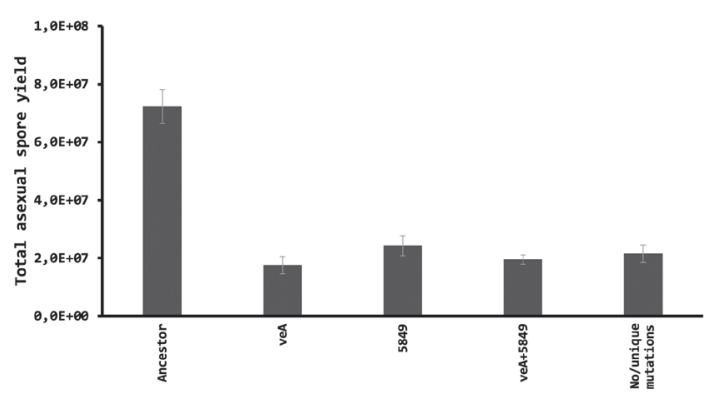

B

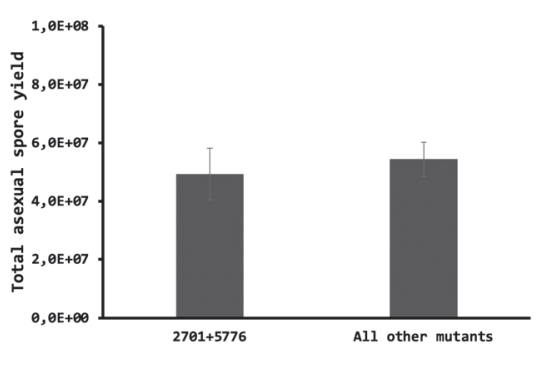

Figure 5.6: Association between genotype and total asexual spore yield. (A) Isolates grouped by the presence of mutant alleles at veA and $A N 5849$, and their combination at week 10. Each bar represents the mean spore yield for each group and error bars are SEM. (B) Double mutants of AN2701 and AN5776 and all other mutants at week 52. Each bar represents the mean spore yield for each group and error bars are SEM. 


\section{Metabolic interaction}

In chapter 4 , we reported that FL mutants reduced the asexual spore yield of $A L$ mutants when they were grown on FL-conditioned medium. This reduction in sporulation was rescued when the conditioned medium was supplemented with nutrients, suggesting that resource competition explained the negative effect of $\mathrm{FL}$ on AL's spore production. veA is a known master regulator of secondary metabolism in $A$. nidulans. Therefore, we tested the possible role of veA mutations in metabolic interaction by grouping the genotypes from week 52 into two groups: presence of mutations in veA (Y3FL, Y3AL, Y4FL, Y4AL, Y5FL, Y5AL, Y6FL, N1AL, N3AL) and absence of mutations in veA (N4FL, N4AL, Y6AL, N1FL, N3FL). Although morphotypes with mutations in veA had a stronger negative effect on spore yield of their competitors, this effect was not significant ( $p>0.05$, figure 5.7). We therefore conclude that veA does not have a significant effect on resource competition influencing spore yield.

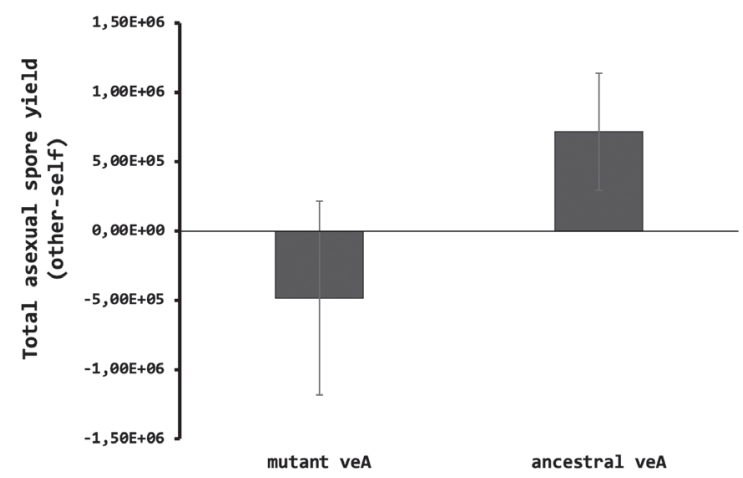

Figure 5.7: Effect of mutations in veA on spore production of the contemporary other morphotype from the final time-point. Each bar shows the average difference of total spore yield on medium conditioned by the other type versus medium conditioned by the genotype itself (without supplementation), which is given separately for medium conditioned by strains having the mutant veA alleles versus those with the ancestral allele. Error bars are SEM.

\section{Sexual reproduction}

Previous work by Kim et al. (2002) had found that deletion of veA abrogates sexual reproduction (i.e. cleistothecia formation). To test the effect of veA mutations on sexual reproduction in our clones, we grouped the week-10 clones into two categories: presence of mutations in veA (N1FL, N3FL, N3AL, N5FL, N5AL, Y5FL, $\mathrm{Y} 6 \mathrm{FL}$ ) and absence of mutations in veA (Y0, N0, N1AL, Y5AL, Y6AL). We found variable results for the effect of veA mutation on sexual spore yield (table 5.2), with a trend towards reduced sexual reproduction in isolates with veA mutations, but the effect is again not significant (Fisher's exact test, $p=0.221$ ) suggesting that mutations in veA do not abrogate sexual reproduction consistently in the ASEX clones. 


\begin{tabular}{|c|c|c|c|c|c|}
\hline & Isolate & Replicate 1 & Replicate 2 & Replicate 3 & Remarks \\
\hline \multirow{7}{*}{$\begin{array}{l}\text { Mutant } \\
\text { veA }\end{array}$} & N1FL & - & - & - & \\
\hline & N3FL & $+/-$ & $+/-$ & $+/-$ & $\begin{array}{l}\text { Very few FB }<5 \% \text { of control. } \\
\text { And FB are }<50 \% \text { of control } \\
\text { diameter. }\end{array}$ \\
\hline & N3AL & + & + & + & \\
\hline & N5FL & + & + & + & \\
\hline & N5AL & + & + & + & \\
\hline & Y5FL & - & $+/-$ & $+/-$ & $\begin{array}{l}\text { Very few FB < } 5 \% \text { of control. } \\
\text { Only FB near inoculum. }\end{array}$ \\
\hline & Y6FL & - & - & - & \\
\hline \multirow{5}{*}{$\begin{array}{l}\text { Ancestral } \\
\text { veA }\end{array}$} & YO & + & + & + & \\
\hline & No & + & + & + & \\
\hline & N1AL & + & + & + & \\
\hline & Y5AL & $+/-$ & $+/-$ & $+/-$ & $\begin{array}{l}\text { Very few FB < } 5 \% \text { of control. } \\
\text { Only FB near inoculum. }\end{array}$ \\
\hline & Y6AL & + & + & + & \\
\hline
\end{tabular}

Table 5.2: Effect of mutations in veA on sexual sporulation in week-10 isolates. + fruiting, - no fruiting, +/- severely reduced fruiting for genotypes with versus without mutations in veA. Mutant veA are strains with mutations in $v e A$ and ancestral $v e A$ have the same sequence as the ancestor. YO and NO are the ancestors. $\mathrm{FB}=$ fruiting bodies

\section{Effect on mycelial fusion}

Fusion between vegetative hyphae of a single colony is essential for intrahyphal communication and homeostasis and fusion between mycelia of different individuals is important for heterokaryon formation, during sexual and para sexual reproduction and non-self and self recognition (Pontecorvo, 1956; Read et al., 2010; Rech et al., 2007). In N. crassa, the soft gene is required for vegetative cell fusion and postfertilization events and fusion defeciency mutants benefit by contributing less to the formation of mycelia, but are over represented during asexual sporulation. (Fleißner et al., 2005; Grum-Grzhimaylo et al., 2021). To test the effect on fusion of mutations in soft and ham-5, we inoculated the final timepoint clones with mutations in soft and ham-5, namely Y2AL (cnx), Y4FL (cnx), N1FL (nia) and N6AL (nia) and the ancestral strains Y0 ( $c n x$ ) and NO (nia) with the wildtype copy of the soft homologue, as described in materials and method. The minimal medium contains nitrate as the only source of nitrogen which cannot be utilized by cnx and nia mutants, so that they will not grow by themselves. If the strains have wildtype fusion abilities and are of the same compatibility type, they will fuse to form a heterokaryon, after which complementation for the cnx and nia mutations occurs. As a result of complementation, the heterokaryon can utilize nitrate and grow on the minimal medium. In total 9 cnx-nia combinations could be made with these strains. All nine combinations were inoculated close to each other on a plate 
in a $3 \times 3$ grid, using three replicate plates. We found that the mutations in the soft homologue is strongly limiting the ability of an isolate to form a heterokaryon with another isolate that has a mutation in the soft homologue (middle and right columns of the grid in figure $5.8 \mathrm{~B}$ and $\mathrm{C}$ ). When an isolate with a mutation in the soft homologue is combined with the ancestor (left column of the grid in figure $5.8 \mathrm{~B}$ and $\mathrm{C}$ ) without the mutation, complementation occurs with a delay, indicating a lower rate of fusion (figure 5.8).

A

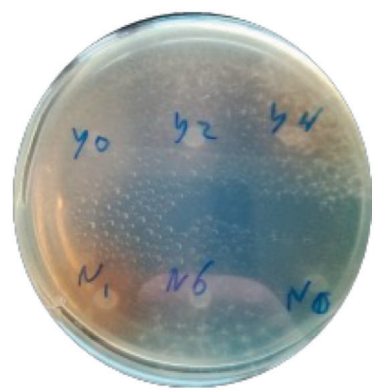

B

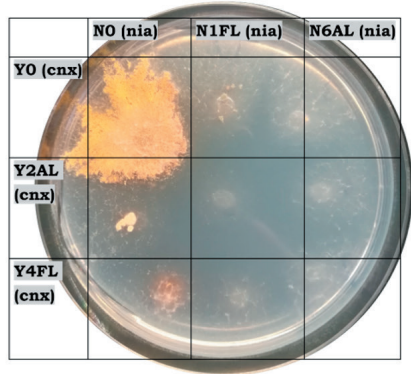

C

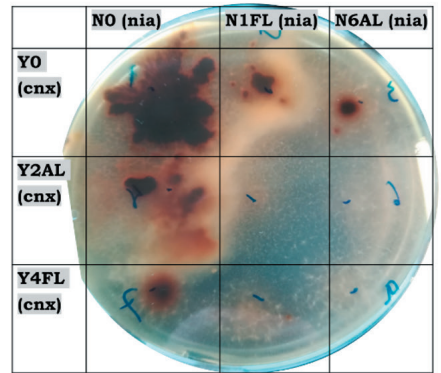

Figure 5.8: Mycelial fusion test of evolved genotypes (N1FL, N6AL, Y2AL and Y4FL) from the final time-point with mutations in the homologues of the soft and ham-5 of Neurospora. (A) Control plate on which each of the strains used for the complementation test is inoculated separately on minimal medium, showing absence of growth. (B) Complementation test after 6 days of incubation. Genotypes affected by mutations in soft homologues (N1FL, N6AL, Y2AL and Y4FL) together show no growth, while mutant soft homologues show very little growth in combination with a wild type (ancestor $\mathrm{YO}$ and $\mathrm{NO}$ ). Wild types for the soft homologue show full complementation indicating fusion. (C) Complementation test after 16 days of incubation. Mutant soft homologues together still show no growth, but they now show growth in combination with a wild type, indicating delayed or reduced fusion.

\section{Discussion}

The $E$. colilong-term evolution experiment (LTEE) (Lenski et al., 1991) inspired our Aspergillus short-term evolution experiment (ASEX). The E. coli populations of the LTEE experiment are evolving in an unstructured minimal-medium environment, where $1 \%$ of each population is transferred daily to fresh medium. ASEX was designed to understand the nature of adaptation of a filamentous fungus to growth on structured minimal nutrient media, where $A$. nidulans was allowed to grow and reproduce asexually for seven days before $1 \%$ of the asexual spores was transferred to fresh medium. The serial transfer regime of the ASEX populations resembles a seasonal environment, with a temporal difference in resource availability. Under such conditions, fungi such as $A$. nidulans are likely to allocate resources to grow to an optimal size and then invest in reproduction without further growth (bangbang strategy). The timing of this strategy depends on resource concentration and utilization rate (Gilchrist et al., 2006). The mass collection and transfer of asexual spores used in our transfer regime destroys population structure at every transfer, 
which limits the evolution of long-term positive social interactions and may select for intra-somatic exploitation strategies resulting in changes in the level of selection from between to within mycelia (Grum-Grzhimaylo et al., 2021).

The whole-genome sequencing of evolved clones from the ASEX populations showed that the transition to transversion ratio is twice as high as expected and the ratio of $\mathrm{dN} / \mathrm{dS}$ across the genome was not significantly different from the neutral expectation, suggesting an important role for genetic drift. However, we also found that mutations in the genes that have been mutated parallelly in multiple populations occurred more frequently than expected when mutations would occur randomly (figure 5.2B), suggesting that they are under positive selection (figure 5.2A). The LTEE populations instead show overall positive selection across the genome as early as 500 generations (Tenaillon et al., 2016). About $98 \%$ of all point mutations were contributed by the populations that evolved a hyper mutator phenotype. In the LTEE populations that retained the wild-type mutation rate, a 17-fold increase in non-synonymous to synonymous mutations was observed during first 500 generations. The non-synonymous to synonymous mutations continued to accumulate at twice the rate of synonymous mutations in the later generations. A similar trend was observed for intergenic substitutions, which presumably alter binding sites for regulatory proteins, suggesting an adaptive role also for intergenic mutations. When compared to mutation accumulation lines, all major classes of mutations, including point mutations and indels, were adaptive (Tenaillon et al., 2016).

We found at least two different morphs in our populations as early as week 10 . One of the clones had an ancestor-like, the other a fluffy-like colony morphology. These two types persisted until the end of the experiment in seven of the 12 populations. Two alternative evolutionary scenarios may explain this polymorphism: i) The repeated rapid evolution of stable polymorphisms via mutations in shared or different genes across populations, or ii) The repeated evolution of similar morphotypes which are present only temporarily and regularly lost again via either drift or selection. The reconstructed phylogenetic trees based on the sequenced genotypes from week 10 and week 52 (figure 5.3), show only one example of a "deep" divergence of both morphotypes (population Y6), consistent with a longterm stable polymorphism, but more often examples of the repeated evolution of one or both morphotypes from unrelated genetic backgrounds (populations $\mathrm{Y} 3$, $\mathrm{Y} 4, \mathrm{Y} 5, \mathrm{~N} 1, \mathrm{~N} 3, \mathrm{~N} 4$ and N5), consistent with their frequent loss and re-evolution. However, the temporary polymorphisms observed in several populations may also result from incomplete sampling, due to either the absence of clearly distinct 
phenotypes or their presence at too low a frequency to be detected by our sampling method.

In spite of the possibility of incomplete sampling, we see a degree of parallel genomic evolution regarding both morphs, with FL morphs showing early parallel adaptation followed by divergence as time progresses, suggesting that $\mathrm{FL}$ phenotypes share a common genetic basis. In contrast, the AL morphs showed more parallel adaptation at the later time-point, indicating a delayed adaptive response, which was possibly triggered by the arrival of the FL morphs. The significantly greater parallel evolution of AL relative to FL morphotypes after week 10 (figure 5.4) suggests that they at least follow different adaptive trajectories, and not simply "catch up" with the FL types later. The emergence of FL morphs has been reported in various species of Aspergillus grown under similar glucose limiting conditions as in our study (Swift et al., 2000; Withers et al., 1994).

We would have expected, that the observed reduction in spore yield of the ASEX populations was due to mutations in core genes involved in asexual sporulation, such as brlA, abaA, and wetA (T. H. Adams et al., 1998; Martinelli \& Clutterbuck, 1971; Mooney \& Yager, 1990; Park \& Yu, 2012; J.-H. Yu, 2010). Alternatively, selection could also have acted on other stages in the complex life cycle of $A$. nidulans, for example via (i) Changes in the timing of initiating sporulation/development (Noble et al., 2016), (ii) Changes in sensing environmental cues necessary for making decisions to fine-tune investments in growth, colony maintenance (via recycling of damaged mycelium) and reproduction (Heaton et al., 2016) and (iii) Changes in secondary metabolism, known to play a role in fungal development and both positive and negative social interactions (Bayram \& Braus, 2012; Hibbing et al., 2010; Keller, 2019).

In contrast to our expectation of mutations in genes involved in conidiation, we see parallel mutations in eight genes, of which two, veA and $A N 5849$, contribute especially to parallelism (figure 5.5). Mutations in veA and AN5849 seem clearly adaptive, since they appear in four populations at an early time-point, and likely went to high frequency by the end of the experiment in five populations, as we picked them up by sequencing random clones (see figure 5.2). An evolution experiment studying adaptation to submerged cultivation in the ascomycete Podospora anserina also found parallel mutations in veA along with prol homologs of $P$. anserina (Kudryavtseva et al., 2019). Thus, suggesting filamentous fungi adapt to laboratory environment by mutating veA. 
veA is a well characterized fungal transcription factor involved in light-dependent regulation of sexual and asexual reproduction and secondary metabolism (Bayram \& Braus, 2012; Calvo, 2008). Deletion of veA in A. nidulans abrogates sexual reproduction and increases asexual reproduction (Kim et al., 2002). We saw that inactivation of veA has no significant effect on sexual reproduction (see table 5.2), and the reduction in asexual spore yield in our populations contradicts the previously reported increase in asexual spore yield and loss of sexual reproduction in the $\Delta v e A$ strains (Kim et al., 2002). Kim et al., (2002) have measured these phenotypes on rich medium, whereas our populations evolved and were tested on low glucose minimal medium. Glucose concentration is known to both increase (high concentration) and decrease (low concentration) conidiation in A. nidulans (T. H. Adams et al., 1998; Han et al., 2003). In A. fumigatus and N. crassa, deletion of the veA ortholog caused reduced conidiation when cultivated on medium supplemented with standard glucose concentrations for the respective species (Bayram et al., 2008; Krappmann et al., 2005). Orthologs of veA are also known to be involved in cell wall integrity, cell surface hydrophobicity, hyphal polarity, hyphal fragmentation and hyperbranching of hyphal tips (Calvo, 2008; Kato et al., 2003; Park \& Yu, 2016). In conclusion, mutations in veA are known to have an effect on both somatic growth and reproduction, but that the sign and extent of these changes varies across species and conditions.

Production of secondary metabolites and conidiation are tightly linked in many ascomycetes (Bayram \& Braus, 2012). Conidiation is also influenced by the ratio of carbon to nitrogen (Han et al., 2003), as well as by the ratio of fatty acids called oxylipins, and veA is known to regulate oxylipin and other secondary metabolite biosynthesis (Calvo, 2008). Any imbalances in these pathways may affect both asexual and sexual reproduction. Reduction in asexual spore yield, which was the most visually evident parallel phenotypic change we found in all our evolved populations, does not seem due to direct selection on genes involved in asexual reproduction, but is more likely a negative pleiotropic effect of adaptive changes in metabolism.

Of the remaining genes, orthologs of $A N 2701$ and $A N 5776$ are $N$. crassa ham-5 and soft genes, respectively. Deletion of ham-5 and soft in $N$. crassa leads to highly reduced hyphal fusion, reduced conidiation and reduced growth rate and a negative frequency-dependent competitive benefit in mixtures with compatible wild-type conidia (Bastiaans et al., 2016; Grum-Grzhimaylo et al., 2021). As the frequency of the fusion-deficient mutant increases, it increases population structure, due to excess fragmentation of the mycelium, which in turn shifts the level of selection from within to between mycelial colonies (Aldabbous et al., 2010; Grum-Grzhimaylo 
et al., 2021). Possibly, our $A$. nidulans AN2701 and AN5776 mutants provide similar frequency-dependent benefits under the ASEX conditions. The fusion tests (figure 5.8) indeed show that $A$. nidulans so and ham-5 homologues are also involved in hyphal fusion.

To summarize, our $A$. nidulans populations adapted to growth on low glucose minimal medium by targeting genes that bring about global changes in secondary metabolism rather than genes that affect specific functions like growth or conidiation. A possible explanation for this could be that in structured environments like ours, competition occurs locally rather than globally, which limits selection for increased resource competitive ability and faster growth (Habets et al., 2007). Moreover, the transfer regime introduces seasonality to the environment, caused by the weekly reduction of primary resources and accumulation of metabolic by-products between transfers. Harvesting all the spores via shaking with saline solution disrupts the population structure at every transfer, resulting in different local competitors every week (Habets et al., 2006; Perfeito et al., 2008; Vasi et al., 1994). In such environments, it may be more beneficial to produce specific compounds to directly affect your neighbor's success (Wloch-Salamon et al., 2008). However, we did not find evidence for interference competition strategies in the ASEX populations. Our data from the conditioned medium experiments in chapter 4 suggest that resource competition between $\mathrm{FL}$ and $\mathrm{AL}$ clones contributes to the reduced sporulation in $A L$ morphs.

Whereas our genomic analyses find clear evidence for a prominent role of selection in the ASEX populations, as well as for a shared genetic basis and different timing of adaptation of AL and FL types, they do not advance a full mechanistic understanding of adaptation of either type. Detailed molecular and biochemical work will be required to understand the physiological effects of mutations in veA, AN5849, so and ham-5 on secondary metabolite profile, asexual and sexual reproduction and mycelial fusion and their selective benefits. This requires the construction of mutants with these mutations individually and in combinations in the ancestral background, and analysis of their effect on fitness components and on competitive fitness. 


$$
6
$$


CHAPTER 6.

General discussion 
This thesis describes the results of experiments on the causes and constraints associated with adaptation in a simple multicellular modular organism, Aspergillus nidulans, with a special focus on the role of trade-offs between somatic growth and reproduction. Understanding fungal life-history strategies (investments in and timing of growth, maintenance and reproduction) will give us insights into how natural selection operates in these ubiquitous organisms with complex life cycles. It also provides insights into mechanisms that promote fungal population establishment, persistence, extinction, and diversification (Pringle \& Taylor, 2002).

I begin this chapter by briefly describing modular organisms, the evolutionary significance of within-individual variation, units of selection, and fungal life-history strategies. Then, I discuss the role of trade-offs between growth and reproduction that may result from local adaptation, and possible short-term adaptive constraints from such trade-offs under controlled environmental conditions. Subsequently, I discuss the phenotypic and genomic basis of adaptation of a laboratory strain of A. nidulans during a year of adaptation to growth and reproduction on solid media with limiting nutrients. I conclude with an outlook.

\section{Modular organisms, individuality, and units of selection}

Modular organisms have no distinct germline and are theoretically immortal. They are composed of autonomous, repetitive, totipotent, multicellular parts of a structural individual (Vuorisalo \& Tuomi, 1986). A structural individual can be defined as a self-maintaining unit that interacts as a unified whole with its environment and can maintain itself and reproduce (Vuorisalo \& Tuomi, 1986). In modular organisms, heritable somatic variation can be propagated by asexual and sexual means. Within and between-individual variation may thus both have direct evolutionary significance (Tuomi \& Vuorisalo, 1989b, 1989a; Vuorisalo \& Tuomi, 1986; L. Yu et al., 2020).

Within-individual variation can arise by genetic changes such as somatic mutations, mitotic recombination, and genome duplications. Variation can also be generated by the fusion or aggregation of genetically distinct individuals, for example, during heterokaryon formation in a fungal mycelium (Pineda-Krch \& Lehtilä, 2004) and the formation of multicellular aggregate in cellular slime molds (Sathe \& Nanjundiah, 2018). Within-individual variation may benefit the individual because synergistic interactions between genetic variants potentially have beneficial consequences (Pineda-Krch \& Lehtilä, 2004). However, within-individual variation may also be harmful, when a particular variant has a benefit within the individual but provides a disadvantage at the individual level, leading to a conflict between levels of selection. 
In modular organisms, each module is totipotent, and within-individual heritable variation has direct evolutionary consequences, as it may be transmitted to the next generation. This property has been exploited to study the different levels at which selection can act. For example, multi-level selection has been tested in the colonial seagrass Zostera marina where the difference in the levels of selection between inter and intra individual variation has been quantified (L. Yu et al., 2020); in Neurospora tetrasperma where the switch in the level of selection between the nuclei in a homokaryon and a heterokaryotic mycelium has been investigated (Meunier et al., 2018) and in N. crassa, the change in the level of selection from within to between mycelia has been observed (Bastiaans et al., 2016; GrumGrzhimaylo et al., 2021).

Within-individual variation threatens the integrity of an individual due to negative consequences of within-individual competition, for example during cancerous growth and cell parasitism. Within-individual competition has been observed in organisms with aggregative multicellularity, like cellular slime molds and myxobacteria (Strassmann et al., 2000; Velicer et al., 2000). Here, during starvation formerly independent cells come together to form a multicellular structure, and only a fraction of the aggregated cells forms the reproductive structure, and the rest form the non-reproductive stalk in cellular slime molds or are autolyzed in myxobacteria. In both Myxococcus xanthus and Dictyostelium discoideum, when genetically different lineages develop together, some lineages do not contribute their fair share to the non-reproductive tissue and are over-represented in the reproductive tissue (Strassmann et al., 2000; Velicer et al., 2000). However, in heterogeneous groups of cellular slime molds, within-individual competition is reduced by trade-offs between different fitness components, like growth and sporulation (Sathe \& Nanjundiah, 2018) and between aggregating and nonaggregating cells (Dubravcic et al., 2014; Tarnita et al., 2015; Wolf et al., 2015).

Within-individual variation in filamentous fungi can arise due to somatic mutation and fusion between genetically different mycelia. In filamentous fungi, the nuclei are units that can cooperate and compete. Competition between genetically different nuclei can occur in their replication rates and transmission into asexual spores (Meunier et al., 2018). Genotypes that have the potential to threaten the integrity of the individual can be maintained in a negative frequency-dependent manner, and as their frequency increases, they can change the level of selection from within mycelia to between mycelia. For example, in $N$. crassa, as the frequency of the cheater mutants increase, they cause fragmentation of the mycelium and increasing population structure changing the level of selection from within to between mycelia (Bastiaans et al., 2016; Grum-Grzhimaylo et al., 2021). 


\section{Trade-offs in fungal life-history traits}

In $A$. nidulans, asexual reproduction can be induced only after a period of vegetative growth (Axelrod, 1972; Noble \& Andrianopoulos, 2013). Fungal growth can be quantified by the radial colony growth rate (mycelial growth rate, MGR) or increase in fungal biomass. When using MGR as a proxy for fitness, it is essential to keep in mind that MGR typically is inversely related to the nutrient richness of the environment; fungal biomass on the other hand increases with nutrient richness. In nutrient-rich environments, a fungal mycelium is typically dense, compact, and highly branched for nutrient exploitation. In contrast, the mycelium is widespread, thin, and less branched for effective nutrient exploration in nutrient-poor environments (Ritz, 1995; Whiteway \& Bachewich, 2005). Fungi can use different strategies to outcompete their competitors, such as via faster growth and monopolization of a larger fraction of resources, or alternatively, by producing a larger number of offspring (higher spore yield) and outcompete the one with fewer offspring (Heaton et al., 2016). In the absence of genetic constraints or covariances between these traits (MGR and asexual sporulation), both can be independent candidates for adaptation (Gifford \& Schoustra, 2013). However, rapid growth and sporulation may conflict because resources are limited, and energy spent on one trait may not be available for the other (Heaton et al., 2016; van Noordwijk \& de Jong, 1986). The timing of reproduction is predicted to reflect life-history optimization in a given environment. For example, small patches of short-lived resources (e.g., dung or rotting fruit) or intense competition is predicted to reduce the timing of sporulation initiation, whereas long-lived resources (e.g., a fallen tree) are likely to favor multiple reproductive events (Gilchrist et al., 2006; Heaton et al., 2016). Table 1.1 in chapter 1 summarizes the various life-history strategies a fungus can employ under different environmental conditions.

The designs of the two evolution experiments described in this thesis have one thing in common; namely, both experiments made use of a structured synthetic minimal medium as the evolution environment. The main differences between them are that in chapter $\mathbf{2}$, the standard carbon concentration of $1 \%$ glucose was used, and the populations grew from a single point of inoculation as a radial colony on agar surface in a Petri dish. At the time of transfer of spores harvested from the entire colony, the colony had not completely occupied the agar surface, and there was room for more growth, i.e., the resource patch was not entirely exhausted. Under these conditions, one might expect the fungus to produce a dense, highly branched mycelium for effective resource exploitation. Since the resource patch is not fully exhausted and in the absence of competition, the timing of sporulation is likely delayed. In contrast, the "A. nidulans Short-term Evolution eXperiment" (ASEX) populations (chapter 3 ) evolved under $10 \mathrm{X}$ reduced carbon supply $(0.1 \%$ 
glucose), and colonies developed from a relatively high density of spores spread on the agar surface.

In chapter 2, we tested for the existence of a trade-off between MGR and asexual spore production in natural isolates of $A$. nidulans. First, we tested for such a tradeoff among 15 natural isolates in four laboratory environments. An observed tradeoff may reflect adaptive specialization due to local adaptation. We did not see any correlation between the two traits measured within each of the four-environments tested. However, we did see a positive correlation between both traits and the nutrient richness of the growth media.

After a short laboratory evolution experiment with a subset of the natural isolates in one of the four environments, we found a consistent antagonistic relationship between MGR and asexual spore yield since five of the six strains tended to improve their growth rate at the expense of spore yield, while one of the six strains improved its spore yield at the expense of MGR. The negative correlation between MGR and spore density approached significance, but not with spore yield, among independently evolved populations. The negative correlation between the MGR and asexual sporulation (spore density) was significant for only one strain. When wild isolates adapt to a laboratory environment, they may shut off the production of energetically expensive extracellular enzymes (Zheng et al., 2020). Turning off unnecessary cellular functions may explain why our strains tended to evolve higher MGR.

The protocol of the selection experiment involved washing off spores from the surface of the colony. Thus, spore yield could be improved by increasing the density of spores on the colony's surface without increasing mycelial growth area. In that case, we would not observe any correlation between the two traits. Alternatively, spore yield may be enhanced by increasing the mycelial surface area for sporulation, in which case we would observe a positive correlation between the traits. However, we observed a negative correlation between spore density and MGR, suggesting that mutations causing increased spore yield were not accessible to evolution (Schoustra \& Punzalan, 2012). If the fungi adapted by changing the timing of sporulation, then delayed sporulation may be interpreted as reduced sporulation if spore yield was quantified before the completion of sporulation. Most of the natural isolates we examined evolving in the laboratory were investing more in exploration (i.e., they increased in growth rate) rather than exploitation (increase in spore yield) life-history strategies, with the exception of one isolate that improved spore yield at the cost of growth suggesting that the optimal MGR is lower under natural conditions. 
Chan et al., (2020) tested the role of patch size and nutrient richness on the timing of reproduction in the lab isolate of the ascomycete fungus Phacidium lacerum. They found that patches with high nutrition supported earlier reproduction than low nutrient patches. Larger patches also supported early reproduction irrespectively of nutrient richness. In comparison, smaller patches supported a bang-bang lifehistory strategy (ref. table 1.1), where the colony first invested resources in the growth and invested resources into reproduction only when growth became limited due to lack of space. They concluded that initiating reproduction depends on the colony's ability to sense its internal nutrient reserve and available external space for growth. However, they also found a negative correlation between growth and spore yield in all conditions tested, suggesting that the timing of reproduction initiation depends on patch size and nutrient richness and the correlation between growth and spore yield is fundamental to the organism's biology (Chan et al., 2020).

In contrast, when a lab isolate of basidiomycete fungus Coprinus cinereus was grown on resource patches of varying sizes and richness, a positive correlation was observed between growth measured as biomass and spore yield on all patch sizes studied. However, when they normalized the growth and spore yield to the richness of the patch, they found that growth and spore yield were negatively correlated only on larger patches with low richness, indicating that increased resource allocation to mycelium came at a cost to reproduction (Schmit, 2002). A different study found no correlation between growth rate and yield among the natural isolates of the ascomycete fungus, $N$. crassa, when cultivated in rich and poor nutrient environments (Anderson et al., 2019). They further experimentally evolved the natural isolates for either growth or spore yield improvements, measured the effect of selection on the non-selected trait, and found that growth and reproduction were not correlated (Anderson et al., 2019). These conflicting results from various studies suggest that variation in life-history strategies and the appearance of a trade-off between growth and reproduction depends on the species' biology and environmental conditions.

In contrast to chapter 2, the ASEX populations (chapter 3 ) evolved under 10X reduced carbon supply ( $0.1 \%$ glucose), and colonies developed from a relatively high density of spores spread on the agar surface. The fungus completely covered the agar's surface within 48 hours, so the resource patch was likely exhausted when spores were transferred after seven days. The spreading of the spores on the medium surface resulted in many starting individual colonies growing and interacting in a limited space. In $A$. nidulans, fusion between conidia is infrequent and not essential for colony establishment and thus colonies remain mostly independent (Read et al., 2010). Due to factors such as limiting resources, crowding 
due to limited space, multiple starting colonies, and disruption of population structure at every transfer, the fungus may evolve strategies to grow faster and occupy the niche to exclude competitors. Alternatively, it may also exclude competitors by engaging in interference competition via toxin production.

In the ASEX populations, we observed a reduction in asexual spore yield and no systematic changes in MGR over evolutionary time. We found a statistically non-significant positive correlation between MGR and spore yield across independently evolved populations at the final time point. Thus, we found no support for a trade-off between growth and reproduction across independently evolved populations. In the long-term evolution experiment with the bacterium Escherichia coli (LTEE) (Lenski et al., 1991), that inspired the ASEX experiment, a positive, statistically non-significant, correlation was found between growth rate and yield across independently evolved populations. This positive correlation was attributed to the fact that the populations were initially ill-adapted to the evolutionary environment. Therefore, the populations improved in both traits as they evolved towards the hypothetical trade-off line (Novak et al., 2006). In single celled organisms' growth and reproduction are synonymous, however, in multicellular organisms such as a filamentous fungus, reproduction can begin only after a period of somatic growth, as somatic tissue is required for the development of reproductive structures. Selection for improvements in growth might also indirectly affect reproduction because of an increase in the somatic biomass required for sporulation. Alternatively, improvements in reproduction can also be achieved without increasing growth rate; for example, by increasing the density of spores per surface area of the somatic tissue. Therefore, for a filamentous fungus, finding trade-offs between adaptive changes in growth and reproduction is complicated by these additional positive dependencies between somatic growth and reproduction.

The parallel reduction in asexual sporulation in the ASEX populations suggests, that reduced spore yield is adaptive in the evolution environment. However, we currently do not understand the nature of this benefit. The spreading of the spores on the agar medium surface produces many individual colonies growing on a small resource patch. Such a condition is likely to cause competition for space and resources. The role of a trade-off between growth and asexual sporulation in the presence of competitors (both interspecific and intraspecific) was tested in P. lacerum by Chan et al., (2019). The authors hypothesized that the presence of competitors would result in early and increased allocation to reproduction; however, they did not find a correlation between the timing of reproduction and the presence of competitors. Interspecific competitors, but not an intraspecific 
competition with clonally related spores, reduced asexual sporulation (Chan et al., 2019). Initially, the interaction between individual colonies in ASEX populations is with genetically similar resembling intraspecific competition. As mutations arise and spread during adaptation, multiple genotypes may coexist and competitively interact resembling interspecific competition.

In both evolution experiments described in this thesis, the evolved populations tended to reduce their asexual spore production. However, the changes in MGR were different between the two experiments (chapters 2 and 3 ). The natural isolates evolved to improve their MGR, whereas the ASEX populations did not change in MGR. In theory, a larger mycelium provides a larger surface for sporulation, and thus both traits should be positively correlated (Gifford \& Schoustra, 2013). However, the density of mycelium differs based on the growth rate. Slower growth results in a dense, highly branched mycelium that may be better at resource capture than faster-growing thin mycelium. MGR is measured by point-inoculating the populations and averaging the growth over a four-day growth period. The differences in how MGR responded to selection in the two experiments are most likely caused by how the fungi were inoculated: either growing from a single point inoculation (chapter 2) or from multiple positions after spreading of spores (chapter 3 ). Using point-inoculation for measuring MGR in ASEX populations may not have captured the contribution of growth to adaptation in the ASEX environment since competition between colonies may be a factor that influences MGR. Faster growth would help occupy a resource patch faster, but may not provide enough biomass for sporulation. In support of this idea, we find that spore density and MGR are negatively correlated (Chapter 2).

\section{Ecological opportunities for adaptive trade-offs and divergence}

Asexual populations evolving in an environment with one limiting resource will adapt through selective sweeps of fitter clones in a sequential manner (Muller, 1932). However, this view of clonal replacements is complicated by clonal interference and genetic hitch-hiking (Cvijović et al., 2018). Numerous studies have reported the spontaneous evolution of stable polymorphisms of at least two genotypes in initially clonal populations (Atwood et al., 1951; Bastiaans et al., 2016; Elena \& Lenski, 1997; Frenkel et al., 2015; Helling et al., 1987; Maddamsetti et al., 2015; Morris et al., 2014; Rainey \& Travisano, 1998; Rozen \& Lenski, 2000; P. E. Turner et al., 1996; Zhang et al., 2017). Adaptive radiation, a process where descendants from a common ancestor rapidly diversify in a new environment by adapting to diverse niches, is frequently observed, and has been attributed to environmental heterogeneity (Gillespie et al., 2001; MacLean, 2005). Niches may arise in nonstructured environments from temporal heterogeneity in a single 
resource (seasonal environment (Vasi et al., 1994)) or an excreted metabolite (cross-feeding on a metabolizable metabolite). In structured environments, niches are created also due to spatial heterogeneities, which allow adaptation by niche specialization and frequency-dependent fitness trade-offs in alternate niches, leading to stable polymorphisms (Rainey et al., 2000; Rainey \& Travisano, 1998).

The competitive fitness of the ASEX populations did not systematically improve compared to the ancestor. A possible explanation for this is that fitness interactions may not be transitive during evolution. Non-transitive fitness interactions means that in an evolutionary succession from an ancestor to an evolved type 1 and from evolved type 1 to evolved type 2, the fitness of type 1 is larger than the fitness of the ancestor, and fitness of type 2 is larger than that of type 1, but still the fitness of type 2 may not be larger than the fitness of the ancestor. Two tested ASEX populations indeed exhibited non-transitive fitness interactions, which we attributed to the evolution of polymorphisms of multiple genotypes with frequency-dependent fitness interactions. Indeed, we found at least two different morphotypes, named fluffy-like (FL) and ancestor-like (AL), as early as week 20 in all the populations. In seven populations, they were present until the end of the experiment. Apart from the difference in their colony morphology, the two morphs did not consistently differ in the fitness components tested, namely MGR or spore yield.

In chapter $\mathbf{4}$ we began by conducting competitions with the two morphotypes at low and high initial frequencies from the final time point, and observed that the FL types from the final time point had an advantage over contemporary AL types when they were introduced at $10 \%$ in the population. The relative fitness of FL types was lower at - $90 \%$ (hence supporting negative frequency dependence), but unexpectedly, they did not experience a fitness disadvantage relative to $\mathrm{AL}$ types at this frequency. Two possible explanations for this observation are: (i) The equilibrium frequency of $F L$ is even higher than $90 \%$, or (ii) The fitness of the two dominant types used for the competitions is dependent on interactions with yet other genotypes present in the population (Maddamsetti et al., 2015). We could exclude the first explanation, because the grand mean frequency at the final time point of FL types was $\sim 50 \%$, which is well within the range of tested frequencies of FL types in the competitions. Hence, we hypothesized that the competition environment failed to recapitulate the relevant evolutionary environment, and additional genotypes present in the evolving populations affect the fitness of FL and/or AL types and contribute to the observed AL/FL polymorphism. 
We explored the possible mechanism involved in maintaining the observed morphological diversity in the populations, by testing the possible role of metabolites and toxins in causing the frequency-dependent fitness interactions. We tested both negative (interference competition) and positive (cross-feeding) metabolic interactions, using conditioned media. We found that the AL morphotype produced fewer spores when cultured on its contemporary FL- conditioned medium compared to its own conditioned medium. In contrast, FL produces relatively more spores on medium conditioned by AL. After supplementation of FL-conditioned medium with the original amount of nutrients, AL's spore yield reduction was reversed. From these experiments, we concluded that FL and AL do not engage in interference competition, but FL is likely a superior resource competitor relative to AL. The depletion of usable nutrients in un-supplemented FL spent medium thus seemed responsible for the lower spore yield of $A L$, and the higher reproduction of FL on AL-conditioned medium could be due to lower depletion of useable nutrients by AL.

Rozen and Lenski (2000) found two stably coexisting morphs engaged in a negatively frequency-dependent interaction in one of the LTEE population. One of the morphs influenced the survival of the other morph by removing essential nutrients in the medium and grew better on the conditioned medium than on fresh medium. Complex mechanisms involving trade-offs between resource competition and death rates in the stationary phase, combined with cannibalism, helped maintain this bacterial diversity (Rozen et al., 2009; Rozen \& Lenski, 2000). In $N$. crassa, the fluffy-like mutants were deficient in somatic fusion and gained an advantage at a low frequency when there was a high probability of wild-type mycelia fusing with them. Under such conditions, fluffy-like mutants were more likely to be over-represented in spores (Grum-Grzhimaylo et al., 2021). In addition to resource competition, we consider it possible that $\mathrm{FL}$ and $\mathrm{AL}$ types in our experiment also engage in intra-somatic interactions which may contribute to their stable co-existence. We expect intra-somatic interaction may be important as it is known to play a role in $N$. crassa during adaptation to a structured environment (Bastiaans et al., 2016; Grum-Grzhimaylo et al., 2021). FL and AL types may also engage in other physical interactions, such as FL type may produce more aerial hyphae that may overgrow and hinder AL types' sporulation. Table 6.1 summarizes the main expectations and findings of this section. 


\section{Natural isolates (Chapter 2)}

Populations adapted as a single expanding individual with space for growth and high carbon (compared to ASEX)

Expectation

Findings
Improvements in both growth and spore yield or in one of the traits

A slight improvement in growth and no effect on spore yield, but a decrease in spore density

\section{ASEX populations (Chapter 3)}

Populations adapted as multiple individuals growing in limited space and on low carbon

Expectation

Findings
Improvements in growth or spore yield via competitive interactions among multiple individuals

No improvements in growth, reduced spore yield and parallel evolution of stable polymorphisms involving resource competition

Table 6.1: Summarizes of the experimental design, expectations and the findings from the two evolution experiments with $A$. nidulans described in this thesis.

\section{Genomic basis of adaptation in ASEX populations}

In Chapter 5, we explored the genomic basis of adaptation of the ASEX populations by focusing on the role and targets of natural selection. We did this by sequencing clones from both morphotypes, as well as clones with undetermined morphotypes, from all 12 populations at two-time points, week 10 (early) and week 52 (final) of the evolution experiment. We tested the role of natural selection in our experiment by comparing the rates of non-synonymous and synonymous substitutions in all the sequenced clones from both time points. The average per-genome ratio of $\mathrm{dN} / \mathrm{dS}$ across our populations was not significantly different from the neutral expectation of 1 , suggesting that a substantial fraction of the observed mutations across the genome were non-adaptive. However, we found that genes mutated in two or more populations occurred more frequently than expected by chance, which suggested that they were under positive selection and furthermore, for those genes the $\mathrm{dN} / \mathrm{dS}$ ratio was larger than 1 . For comparison, the LTEE populations showed a clear positive selection across the genome as early as 500 generations, which continued to 50,000 generations (the final time point analyzed), implying that most non-synonymous mutations were adaptive even after 50,000 generations in a constant environment (Tenaillon et al., 2016).

In seven of the 12 ASEX populations, the two morph types persisted until the end of the experiment. In only one population, these morphotypes showed deep divergence, where the morphotypes isolated at week 10 have persisted and evolved as separate clades until the end of the experiment (figure 5.3. chapter 5). However, more frequently we saw the repeated evolution of the morphs from earlier genotypes with a different colony morphology, suggesting their frequent 
extinction and re-evolution from different genotypes. The finding of temporary polymorphisms could also be due to incomplete sampling, caused by the absence of clearly distinct phenotypes at the time of sampling. Despite frequent reevolution of the morphotypes, we saw significant parallel genomic evolution among isolates from different populations belonging to the same morphotype, which suggests a common genetic basis. The FL morphs showed early parallel adaptation followed by divergence as time progressed. AL morphs, in contrast, showed parallel adaptation at the later time-point, indicating a delayed adaptive response after the arrival of the FL morphs.

For comparison, the bacterial $\mathrm{L}$ and $\mathrm{S}$ morphs that evolved in population Ara2 of the LTEE experiment (Rozen \& Lenski, 2000) have been stably coexisting by adaptive specialization to their respective niches in each other's presence (Le Gac et al., 2012). Genomic analysis showed that $S$ morphs arose from a single ancestral $S$ clone, whereas most of the $L$ morphs were monophyletic and some belonged to a third clade, and there was continuous adaptive evolution of the two types (Rozen et al., 2005).

Of the eight parallel mutated genes in our populations, veA and orthologs of AN2701 and AN5776 are well characterized in A. nidulans and N. crassa, respectively. $v e A$ is a fungal transcription factor involved in the light-dependent regulation of sexual and asexual reproduction and secondary metabolism by acting as a scaffold for interaction with many proteins involved in secondary metabolism (Bayram \& Braus, 2012; Calvo, 2008). The veA-inactivating mutations of the ASEX clones did not change the asexual spore yield compared to clones without veA mutations and showed variation in their ability to form sexual spores (see Table 5.2, Chapter 5). Previous work has shown that deletion of veA in $A$. nidulans abrogates sexual reproduction and increases asexual reproduction (Kim et al., 2002). In the study by Kim et al. (2002), the veA gene was inactivated by the insertion of a large DNA fragment, and asexual sporulation was quantified on complete medium and minimal medium with $1 \%$ lactose as single carbon source. However, the spore yield of the ASEX clones has been quantified on minimal medium with $0.1 \%$ glucose as single carbon source (the evolution environment), and the veA-inactivating mutations are either point mutations or small indels. The differences in the growth medium, the nature of veA mutations, and the presence of other gene mutations in our study might be responsible for the differences in the observed results. Reduction in asexual spore yield in all our evolved populations is more likely due to negative pleiotropic effects of adaptive changes in metabolism. The deletion of orthologs of veA in other filamentous fungi like $A$. fumigatus and $N$. crassa is known 
to reduce conidiation; these contrasting results suggest that the variable effects of veA mutations on asexual sporulation are species-specific.

The orthologs of two other parallel mutated genes, AN2701 and AN5776, are the well-characterized $N$. crassa ham-5 and soft genes, respectively. Deleting ham5 and soft in $N$. crassa leads to reduced hyphal fusion, reduced conidiation and growth rate, and a negative frequency-dependent competitive benefit in mixtures with compatible wild-type conidia (Bastiaans et al., 2016; Grum-Grzhimaylo et al., 2021). The fusion tests of our populations (Chapter 5 ) showed that mutations in $A$. nidulans $A N 2701$ and $A N 5776$ also reduce hyphal fusion. The role of reduced fusion in a competitive (possibly frequency-dependent) benefit in our ASEX populations, however, needs to be established and its mechanism further investigated.

\section{Conclusion}

Trade-offs between life-history traits, an important driving force in constraining adaptation and maintaining diversity, have been empirically tested in plants, animals and microbes, but relatively little in filamentous fungi (Bono et al., 2017; Ferenci, 2016; Roff, 2000; Stearns, 1989). Fungi are ubiquitously distributed and make up large fraction of the Earth's biomass, and understanding their life-history strategies will give us insights into how ecology affects the constraints among various fitness components in these modular multicellular organisms.

We tested for trade-offs between mycelium growth rate and asexual spore production in $A$. nidulans using natural isolates and evolved populations from labadapted strains. We tested for trade-offs using two approaches: by measuring changes in the two traits over evolutionary time (for the lab-adapted strains, chapter 3 ) and across independently evolved replicate populations (for both the natural isolates and lab-evolved strains in chapter $\mathbf{2}$ and $\mathbf{3}$ ). In both cases, we found no clear trade-offs between mycelial growth rate and asexual reproduction.

One other study that tested for a trade-off between growth and reproduction in natural isolates of the filamentous fungus $N$. crassa, found no correlation between mycelium growth and spore yield (Anderson et al., 2019). In contrast, in the basidiomycete fungus $C$. cinereus, a trade-off between growth (measured in terms of biomass increase) and fruiting body production was observed only in low resource conditions (Schmit, 2002). In another study with a laboratory strain of $A$. nidulans, a negative correlation was found between growth rate and spore density (Schoustra \& Punzalan, 2012). The varying results between the studies for different species suggest that variation in life-history strategies and trade-offs among the fitness 
components involved, may be species specific. On the other hand, if the populations are far from a fundamental trade-off line and their growth rate and spore yield are very low when compared to what they can achieve when they reach the trade-off line, then we may not observe a negative correlation (Novak et al., 2006).

In structured environments, in the absence of dispersal, mutants compete mostly with their clone mates, which prevents differences in resource competitive ability to be realized effectively. Adaptive genotypes (in the form of radially growing colonies) can only realize their resource competitive superiority at the perimeter of the colony where they compete against inferior genotypes. Along with spatial structure, the ASEX populations also experienced disruption of population structure during mass harvesting of spores before transfer. Such disturbances will limit long-term social interactions between local neighboring genotypes, possibly favoring interference competition strategies via the production of anti-competitor toxins (Wloch-Salamon et al., 2008) or intra-somatic exploitation strategies that change the level of selection from within to between mycelial colonies (GrumGrzhimaylo et al., 2021; Meunier et al., 2018).

While adapting to a simple, well-defined structured environment, the ASEX fungal populations evolved unexpectedly parallel polymorphisms involving at least two conspicuous morphotypes, between which there were frequency-dependent fitness interactions. We saw that natural selection played a role during laboratory evolution, albeit on specific genes and not across the whole genome. Out of the eight parallel targets of selection, one, namely veA, is involved in fungal secondary metabolism; and two others, namely so and ham-5, are involved in mycelial fusion. We also found evidence for a shared genetic basis and different timing of adaptation of AL and FL types. Nevertheless, we currently lack a mechanistic understanding of the involvement of secondary metabolism and/or mycelial fusion in adaptation of either morphotype.

\section{Outlook}

Fungi inhabit a wide range of ecological conditions and have an essential role in ecology, medicine and agriculture. Therefore, understanding their adaptive strategies, including possible constraints from how their fitness traits are correlated, is highly valuable - particularly, because fungi are little used as model systems in evolutionary studies. Generally, the fitness of fungi is inferred based on measurements of a few convenient traits, such as MGR and spore yield, which are thought to correlate with fitness, and choices made by the experimenter between these traits are made based on knowledge of organismal biology (Pringle \& Taylor, 
2002). However, our work and that of others (Anderson et al., 2019; Damialis et al., 2015; Schmit, 2002) suggests that finding meaningful proxies of fitness might be difficult for filamentous fungi, more so without a robust knowledge of fitness components of a particular species. Ours and the studies mentioned above stress the importance and challenges of understanding the causes and constraints of adaptation of fungi to varying environmental conditions. Nevertheless, laboratory evolution experiments under controlled conditions, like the ones reported in this thesis, are a valuable tool to advance this understanding.

Fungal secondary metabolites have a widespread application in human health and nutrition, such as antibiotics like penicillin and toxins like aflatoxin, as well as in plant physiology and health, such as virulence factors produced during infection (Keller, 2019; Macheleidt et al., 2016; Martin \& Gutierrez, 1992). Our finding that A. nidulans repeatedly adapts to a simple laboratory environment via mutations affecting veA, a master positive regulator of secondary metabolism and somatic fusion, suggests an important role for these during adaptation to growth in a minimal carbon environment. 

References 
Acerenza, L. (2016). Constraints, Trade-offs and the Currency of Fitness. Journal of Molecular Evolution, 82(2-3), 117-127.

Adams, J., \& Rosenzweig, F. (2014). Experimental microbial evolution: History and conceptual underpinnings. Genomics, 104(6), 393-398.

Adams, T. H., Wieser, J. K., \& Yu, J.-H. (1998). Asexual Sporulation in Aspergillus nidulans. Microbiology and Molecular Biology Reviews, 62(2), 545-545.

Aldabbous, M. S., Roca, M. G., Stout, A., Huang, I. C., Read, N. D., \& Free, S. J. (2010). The ham5, rcm-1 and rco-1 genes regulate hyphal fusion in Neurospora crassa. Microbiology, 156(9), 2621-2629.

Anderson, J. L., Nieuwenhuis, B. P. S., \& Johannesson, H. (2019). Asexual reproduction and growth rate: independent and plastic life history traits in Neurospora crassa. ISME Journal, 13(3), 780-788.

Atwood, K. C., Schneider, L. K., \& Ryan, F. J. (1951). Periodic Selection in Escherichia Coli. Proceedings of the National Academy of Sciences, 37(3), 146-155.

Avrahami-Moyal, L., Engelberg, D., Wenger, J. W., Sherlock, G., \& Braun, S. (2012). Turbidostat culture of Saccharomyces cerevisiae W303-1A under selective pressure elicited by ethanol selects for mutations in SSD1 and UTH1. FEMS Yeast Research, 12(5), 521-533.

Axelrod, D. E. (1972). Kinetics of Differentiation of Conidiophores and Conidia by Colonies of Aspergillus nidulans. Journal of General Microbiology, 73, 181-184.

Ayala, F. J. (2007). Darwin's greatest discovery: Design without designer. In the Light of Evolution, 1, 3-21.

Bailey, S. F., Blanquart, F., Bataillon, T., \& Kassen, R. (2017). What drives parallel evolution?: How population size and mutational variation contribute to repeated evolution. BioEssays, 39(1), 1-9.

Bailey, S. F., Rodrigue, N., \& Kassen, R. (2015). The effect of selection environment on the probability of parallel evolution. Molecular Biology and Evolution, 32(6), 1436-1448.

Baker, S. E., \& Bennett, J. W. (2007). An Overview of the Genus Aspergillus. In G. H. Goldman \& S. A. Osmani (Eds.), The Aspergilli (pp. 3-11). CRC Press, Taylor and Francis Group.

Barrick, J. E., \& Lenski, R. E. (2013). Genome dynamics during experimental evolution. Nature Reviews Genetics, 14, 827-839.

Bastiaans, E., Debets, A. J. M., \& Aanen, D. K. (2016). Experimental evolution reveals that high relatedness protects multicellular cooperation from cheaters. Nature Communications, 7(May), 1-10.

Bayram, O., \& Braus, G. H. (2012). Coordination of secondary metabolism and development in fungi: The velvet family of regulatory proteins. FEMS Microbiology Reviews, 36(1), 1-24.

Bayram, O., Krappmann, S., Seiler, S., Vogt, N., \& Braus, G. H. (2008). Neurospora crassa ve-1 affects asexual conidiation. Fungal Genetics and Biology, 45(2), 127-138.

Beaumont, H. J. E., Gallie, J., Kost, C., Ferguson, G. C., \& Rainey, P. B. (2009). Experimental evolution of bet hedging. Nature, 462(7269), 90-93. 
Becker, U., Colling, G., Dostal, P., Jakobsson, A., \& Matthies, D. (2006). Local adaptation in the monocarpic perennial Carlina vulgaris at different spatial scales across Europe. Oecologia, 150(3), 506-518.

Bell, G., \& Reboud, X. (1997). Experimental evolution in Chlamydomonas II. Genetic variation in strongly contrasted environments. Heredity, 78(5), 498-506.

Berngruber, T. W., Lion, S., \& Gandon, S. (2015). Spatial Structure, Transmission Modes and the Evolution of Viral Exploitation Strategies. PLoS Pathogens, 11(4), 1-13.

Bertranda, R. L. (2019). Lag phase is a dynamic, organized, adaptive, and evolvable period that prepares bacteria for cell division. Journal of Bacteriology, 201(7), 1-21.

Bleu, J., Gamelon, M., \& Sæther, B. E. (2016). Reproductive costs in terrestrial male vertebrates: Insights from bird studies. Proceedings of the Royal Society B: Biological Sciences, 283(1823), 1-9.

Blount, Z. D., Barrick, J. E., Davidson, C. J., \& Lenski, R. E. (2012). Genomic analysis of a key innovation in an experimental Escherichia coli population. Nature, 489(7417), 513-518.

Blount, Z. D., Borland, C. Z., \& Lenski, R. E. (2008). Historical contingency and the evolution of a key innovation in an experimental population of Escherichia coli. Proceedings of the National Academy of Sciences of the United States of America, 105(23), 7899-7906.

Boggs, C. L. (2009). Understanding insect life histories and senescence through a resource allocation lens. Functional Ecology, 23(1), 27-37.

Bolger, A. M., Lohse, M., \& Usadel, B. (2014). Trimmomatic: A flexible trimmer for Illumina sequence data. Bioinformatics, 30(15), 2114-2120.

Bono, L. M., Smith, L. B., Pfennig, D. W., \& Burch, C. L. (2017). The emergence of performance trade-offs during local adaptation: insights from experimental evolution. Molecular Ecology, 26(7), 1720-1733.

Brasier, C. M. (1970). Variation in a Natural Population of Schizophyllum commune. The American Naturalist, 104(936), 191-204.

Bronikowski, A. M., Bennett, A. F., \& Lenski, R. E. (2001). Evolutionary Adaptation to Temperature . VIII . Effects of Temperature on Growth Rate in Natural Isolates of Escherichia coli and Salmonella enterica from Different Thermal Environments. Evolution, 55, 33-40.

Bruggeman, J., Debets, A. J. M., Wijngaarden, P. J., DeVisser, J. A. G. M., \& Hoekstra, R. F. (2003). Sex slows down the accumulation of deleterious mutations in the homothallic fungus Aspergillus nidulans. Genetics, 164(2), 479-485.

Bryson, V., \& Szybalski, W. (1952). Microbial Selection. Science, 116(3003), 45-51.

Burger, J. R., Hou, C., \& Brown, J. H. (2019). Toward a metabolic theory of life history. Proceedings of the National Academy of Sciences of the United States of America, 116(52), 26653-26661.

Buskirk, S. W., Rokes, A. B., \& Lang, G. I. (2020). Adaptive evolution of nontransitive fitness in yeast. ELife, 9, 1-27. 
Calvo, A. M. (2008). The VeA regulatory system and its role in morphological and chemical development in fungi. Fungal Genetics and Biology, 45(7), 1053-1061.

Chan, J. Y., Bonser, S. P., Powell, J. R., \& Cornwell, W. K. (2019). When to cut your losses: Dispersal allocation in an asexual filamentous fungus in response to competition. Ecology and Evolution, 9(7), 4129-4137.

Chan, J. Y., Bonser, S. P., Powell, J. R., \& Cornwell, W. K. (2020). Environmental cues for dispersal in a filamentous fungus in simulated islands. Oikos, 129(7), 1084-1092.

Chao, L., \& Levin, B. R. (1981). Structured habitats and the evolution of anticompetitor toxins in bacteria. Proceedings of the National Academy of Sciences of the United States of America, 78(10 I), 6324-6328.

Charlesworth, B., \& Charlesworth, D. (2003). Evolution: A Very Short Introduction. Oxford University Press.

Charnov, E. L., \& Berrigan, D. (1991). Evolution of life history parameters in animals with indeterminate growth, particularly fish. Evolutionary Ecology, 5(1), 63-68.

Cingolani, P., Platts, A., Wang, L. L., Coon, M., Nguyen, T., Wang, L., Land, S. J., Lu, X., \& Ruden, D. M. (2012). A program for annotating and predicting the effects of single nucleotide polymorphisms, SnpEff: SNPs in the genome of Drosophila melanogaster strain w1118; iso-2; iso-3. Fly, 6(2), 80-92.

Clark, T. A., \& Anderson, J. B. (2004). Dikaryons of the basidiomycete fungus Schizophyllum commune: Evolution in long-term culture. Genetics, 167(4), 1663-1675.

Coenen, A., Croft, J. H., Slakhorst, M., Debets, F., \& Hoekstra, R. (1996). Mitochondrial inheritance in Aspergillus nidulans. Genetical Research, 67(2), 93-100.

Conrad, T. M., Frazier, M., Joyce, A. R., Cho, B. K., Knight, E. M., Lewis, N. E., Landick, R., \& Palsson, B. (2010). RNA polymerase mutants found through adaptive evolution reprogram Escherichia coli for optimal growth in minimal media. Proceedings of the National Academy of Sciences of the United States of America, 107(47), 20500-20505.

Conrad, T. M., Lewis, N. E., \& Palsson, B. O. (2011). Microbial laboratory evolution in the era of genome-scale science. In Molecular Systems Biology (Vol. 7).

Cooper, T. F., \& Lenski, R. E. (2010). Experimental evolution with E. coli in diverse resource environments. I. Fluctuating environments promote divergence of replicate populations. BMC Evolutionary Biology, 10(1).

Cooper, V. S., \& Lenski, R. E. (2000). The population genetics of ecological specialization in evolving Escherichia coli populations. Nature, 407(6805), 736-739.

Cooper, V. S., Staples, R. K., Traverse, C. C., \& Ellis, C. N. (2014). Parallel evolution of small colony variants in Burkholderia cenocepacia biofilms. Genomics, 104(6), 447-452.

Cvijović, I., Nguyen Ba, A. N., \& Desai, M. M. (2018). Experimental Studies of Evolutionary Dynamics in Microbes. Trends in Genetics, 34(9), 693-703.

Czárán, T., Hoekstra, R. F., \& Aanen, D. K. (2014). Selection against somatic parasitism can maintain allorecognition in fungi. Fungal Genetics and Biology : FG \& B, 73, 128-137. 
D'Souza, G., \& Kost, C. (2016). Experimental Evolution of Metabolic Dependency in Bacteria. PLoS Genetics, 12(11), 1-27.

D'Souza, G., Shitut, S., Preussger, D., Yousif, G., Waschina, S., \& Kost, C. (2018). Ecology and evolution of metabolic cross-feeding interactions in bacteria. Natural Product Reports, 35(5), 455-488.

Damialis, A., Mohammad, A. B., Halley, J. M., \& Gange, A. C. (2015). Fungi in a changing world: growth rates will be elevated, but spore production may decrease in future climates. International Journal of Biometeorology, 59(9), 1157-1167.

Damuth, J., \& Heisler, I. L. (1988). Alternative formulations of multilevel selection. Biology and Philosophy, 3(4), 407-430.

De Visser, J. A. G. M., Hoekstra, R. F., \& van den Ende, H. (1997). Test of interaction between genetic markers that affect fitness in Aspergillus niger. Evolution, 51(5), 1499-1505.

De Visser, J. A. G. M., \& Lenski, R. E. (2002). Long-term experimental evolution in Escherichia coli. XI. Rejection of non-transitive interactions as cause of declining rate of adaptation. BMC Evolutionary Biology, 2(19).

De Visser, J. A. G. M., Zeyl, C. W., Gerrish, P. J., Blanchard, J. L., \& Lenski, R. E. (1999). Diminishing returns from mutation supply rate in asexual populations. Science, 283(5400), 404-406.

Dettman, J. R., Anderson, J. B., \& Kohn, L. M. (2008). Divergent adaptation promotes reproductive isolation among experimental populations of the filamentous fungus Neurospora. BMC Evolutionary Biology, 8(1), 1-14.

Dubravcic, D., van Baalen, M., \& Nizak, C. (2014). An evolutionarily significant unicellular strategy in response to starvation stress in Dictyostelium social amoebae. F1000Research, 3(0), 133.

Dykhuizen, D. E., \& Hartl, D. L. (1983). Selection in chemostats. Microbiology Reviews, 47(2), 150-168.

Eisfeldt, J., Vezzi, F., Olason, P., Nilsson, D., \& Lindstrand, A. (2017). TIDDIT, an efficient and comprehensive structural variant caller for massive parallel sequencing data. F1000Research, 6(0), 664.

Elena, S. F., \& Lenski, R. E. (1997). Long-Term Experimental Evolution in Escherichia coli. VII. Mechanisms Maintaining Genetic Variability Within Populations. In Evolution (Vol. 51, Issue 4, pp. 1058-1067).

Elena, S. F., \& Lenski, R. E. (2003). Evolution experiments with microorganisms: The dynamics and genetic bases of adaptation. Nature Reviews Genetics, 4(6), 457-469.

Engel, T., Verweij, P. E., van den Heuvel, J., Wangmo, D., Zhang, J., Debets, A. J. M., \& Snelders, E. (2020). Parasexual recombination enables Aspergillus fumigatus to persist in cystic fibrosis. ERJ Open Research, 6(00020-2020).

Fabian, D., \& Flatt, T. (2012). Life History Evolution. Nature Education Knowledge, 3(10), 24.

Fagerström, T., Briscoe, D. A., \& Sunnucks, P. (1998). Evolution of mitotic cell-lineages in multicellular organisms. Trends in Ecology and Evolution, 13(3), 117-120. 
Ferenci, T. (2016). Trade-off Mechanisms Shaping the Diversity of Bacteria. Trends in Microbiology, 24(3), 209-223.

Fisher, K. J., \& Lang, G. I. (2016). Experimental evolution in fungi: An untapped resource. Fungal Genetics and Biology, 94, 88-94.

Flatt, T. (2011). Survival costs of reproduction in Drosophila. Experimental Gerontology, 46(5), 369-375.

Fleißner, A., Sarkar, S., Jacobson, D. J., Roca, M. G., Read, N. D., \& Glass, N. L. (2005). The so locus is required for vegetative cell fusion and postfertilization events in Neurospora crassa. Eukaryotic Cell, 4(5), 920-930.

Flynn, K. M., Dowell, G., Johnson, T. M., Koestler, B. J., Waters, C. M., \& Cooper, V. S. (2016). Evolution of ecological diversity in biofilms of Pseudomonas aeruginosa by altered cyclic diguanylate signaling. Journal of Bacteriology, 198(19), 2608-2618.

Folse III, H. J., \& Roughgarden, J. (2010). What is an Individual Organism? A Multilevel Selection perspective. The Quarterly Review of Biology, 85(4), 447-472.

Fong, S. S., \& Palsson, B. (2004). Metabolic gene-deletion strains of Escherichia coli evolve to computationally predicted growth phenotypes. Nature Genetics, 36(10), 1056-1058.

France, M. T., \& Forney, L. J. (2019). The Relationship between Spatial Structure and the Maintenance of Diversity in Microbial Populations. The American Naturalist, 193(4), 503-513.

Frenkel, E. M., McDonald, M. J., Van Dyken, J. D., Kosheleva, K., Lang, G. I., \& Desai, M. M. (2015). Crowded growth leads to the spontaneous evolution of semistable coexistence in laboratory yeast populations. Proceedings of the National Academy of Sciences of the United States of America, 112(36), 11306-11311.

Friesen, M. L., Saxer, G., Travisano, M., \& Doebeli, M. (2004). Experimental evidence for sympatric ecological diversification due to frequency-dependent competition in Escherichia coli. Evolution, 58(2), 245-260.

Friman, V. P., \& Buckling, A. (2013). Effects of predation on real-time host-parasite coevolutionary dynamics. Ecology Letters, 16(1), 39-46.

Friman, V. P., Guzman, L. M., Reuman, D. C., \& Bell, T. (2015). Bacterial adaptation to sublethal antibiotic gradients can change the ecological properties of multitrophic microbial communities. Proceedings of the Royal Society B: Biological Sciences, 282(1806).

Gerrish, P. J., \& Lenski, R. E. (1998). The fate of competing beneficial mutations in an asexual population. Genetica, 127, 127-144.

Gifford, D. R., \& Schoustra, S. E. (2013). Modelling colony population growth in the filamentous fungus Aspergillus nidulans.pdf. Journal of Theoretical Biology, 320, 124130.

Gifford, D. R., Schoustra, S. E., \& Kassen, R. (2011). The length of adaptive walks is insensitive to starting fitness in aspergillus nidulans. Evolution, 65(11), 3070-3078.

Gilchrist, M. A., Sulsky, D. L., \& Pringle, A. (2006). Identifying Fitness and Optimal Life-History Strategies for an Asexual Filamentous Fungus. In Evolution (Vol. 60, Issue 5, p. 970). 
Gillespie, R. G., Roderick, G. K., \& Howarth, F. G. (2001). Adaptive Radiation. Encyclopedia of Biodiversity: Second Edition, 1(ii), 21-36.

Good, B. H., McDonald, M. J., Barrick, J. E., Lenski, R. E., \& Desai, M. M. (2017). The dynamics of molecular evolution over 60,000 generations. Nature, 551(7678), 45-50.

Gorter, F. A., Aarts, M. M. G., Zwaan, B. J., \& de Visser, J. A. G. M. (2015). Dynamics of adaptation in experimental yeast populations exposed to gradual and abrupt change in heavy metal concentration. American Naturalist, 187(1), 110-119.

Graham, J. K., Smith, M. L., \& Simons, A. M. (2014). Experimental evolution of bet hedging under manipulated environmental uncertainty in Neurospora Crassa. Proceedings of the Royal Society B: Biological Sciences, 281(1787).

Grosberg, R. K., \& Strathmann, R. R. (1998). One cell, two cell, red cell, blue cell: The persistence of a unicellular stage in multicellular life histories. Trends in Ecology \& Evolution, 13.

Grosberg, R. K., \& Strathmann, R. R. (2007). The Evolution of Multicellularity :A Minor- Major Transition? Annual Review of Ecology, Evolution and Systematics, 38, 621-654.

Grum-Grzhimaylo, A. A., Bastiaans, E., van den Heuvel, J., Berenguer Millanes, C., Debets, A. J. M., \& Aanen, D. K. (2021). Somatic deficiency causes reproductive parasitism in a fungus. Nature Communications, 12(1), 1-9.

Habets, M. G. J. L., Czárán, T., Hoekstra, R. F., \& De Visser, J. A. G. M. (2007). Spatial structure inhibits the rate of invasion of beneficial mutations in asexual populations. Proceedings of the Royal Society B: Biological Sciences, 274(1622), 2139-2143.

Habets, M. G. J. L., Rozen, D. E., Hoekstra, R. F., \& De Visser, J. A. G. M. (2006). The effect of population structure on the adaptive radiation of microbial populations evolving in spatially structured environments. Ecology Letters, 9, 1041-1048.

Hall, A. R., Scanlan, P. D., Morgan, A. D., \& Buckling, A. (2011). Host-parasite coevolutionary arms races give way to fluctuating selection. Ecology Letters, 14(7), 635-642.

Han, K.-H., Lee, D. B., Kim, J. H., Kim, M. S., Han, K. Y., Kim, W. S., Park, Y. S., Kim, H. B., \& Han, D. M. (2003). Environmental factors affecting development of Aspergillus nidulans. Journal of Microbiology, 41(1), 34-40.

Hansen, S. K., Rainey, P. B., Haagensen, J. A. J., \& Molin, S. (2007). Evolution of species interactions in a biofilm community. Nature, 445(7127), 533-536.

Hardin, G. (1960). The Competitive Exclusion Principle. Science, 131(3409), 1292-1297.

Harshman, L. G., \& Zera, A. J. (2007). The cost of reproduction: the devil in the details. Trends in Ecology and Evolution, 22(2), 80-86.

Heaton, L. L. M., Jones, N. S., \& Fricker, M. D. (2016). Energetic Constraints on Fungal Growth. The American Naturalist, 187(2), E27-E40.

Helling, R. B., Vargas, C. N., \& Adams, J. (1987). Evolution of Escherichia coli during growth in a constant environment. Genetics, 116(3), 349-358.

Hereford, J. (2009). A Quantitative Survey of Local Adaptation and Fitness TradeDOffs. The American Naturalist, 173(5), 579-588. 
Hibbing, M. E., Fuqua, C., Parsek, M. R., \& Peterson, S. B. (2010). Bacterial competition: Surviving and thriving in the microbial jungle. Nature Reviews Microbiology, 8(1), 15-25. Jousset, A., Eisenhauer, N., Merker, M., Mouquet, N., \& Scheu, S. (2016). High functional diversity stimulates diversification in experimental microbial communities. Science Advances, 2(6).

Kassen, R. (2019). Experimental Evolution of Innovation and Novelty. Trends in Ecology \& Evolution, 34(8), 712-722.

Kato, N., Brooks, W., \& Calvo, A. M. (2003). The Expression of Sterigmatocystin and Penicillin Genes in. American Society for Microbiology, 2(6), 1178-1186.

Kawecki, T. J., \& Ebert, D. (2004). Conceptual issues in local adaptation. Ecology Letters, $7(12), 1225-1241$.

Keller, N. P. (2019). Fungal secondary metabolism: regulation, function and drug discovery. Nature Reviews Microbiology, 17(3), 167-180.

Kerr, B., Riley, M. A., Feldman, M. W., \& Bohannan, B. J. M. (2002). Local dispersal promotes biodiversity in a real-life game of rock-paper-scissors. Nature, 418(6894), 171-174.

Ketola, T., \& Saarinen, K. (2015). Experimental evolution in fluctuating environments: Tolerance measurements at constant temperatures incorrectly predict the ability to tolerate fluctuating temperatures. Journal of Evolutionary Biology, 28(4), 800-806.

Kim, H. S., Han, K. Y., Kim, K. J., Han, D. M., Jahng, K. Y., \& Chae, K. S. (2002). The veA gene activates sexual development in Aspergillus nidulans. Fungal Genetics and Biology, $37(1), 72-80$.

Knaus, B. J., \& Grünwald, N. J. (2017). vcfr: a package to manipulate and visualize variant call format data in R. Molecular Ecology Resources, 17(1), 44-53.

Koboldt, D. C., Chen, K., Wylie, T., Larson, D. E., McLellan, M. D., Mardis, E. R., Weinstock, G. M., Wilson, R. K., \& Ding, L. (2009). VarScan: Variant detection in massively parallel sequencing of individual and pooled samples. Bioinformatics, 25(17), 2283-2285.

Krappmann, S., Bayram, O., \& Braus, G. H. (2005). Deletion and allelic exchange of the Aspergillus fumigatus veA locus via a novel recyclable marker module. Eukaryotic Cell, 4(7), 1298-1307.

Kudryavtseva, O. A., Safina, K. R., Vakhrusheva, O. A., Logacheva, M. D., Penin, A. A., Neretina, T. V., Moskalenko, V. N., Glagoleva, E. S., Bazykin, G. A., Kondrashov, A. S., \& Vision, T. (2019). Genetics of Adaptation of the Ascomycetous Fungus Podospora anserina to Submerged Cultivation. Genome Biology and Evolution, 11(10), 2807-2817.

LaCroix, R. A., Sandberg, T. E., O’Brien, E. J., Utrilla, J., Ebrahim, A., Guzman, G. I., Szubin, R., Palsson, B. O., \& Feist, A. M. (2015). Use of adaptive laboratory evolution to discover key mutations enabling rapid growth of Escherichia coli K-12 MG1655 on glucose minimal medium. Applied and Environmental Microbiology, 81(1), 17-30.

Le Gac, M., Plucain, J., Hindré, T., Lenski, R. E., \& Schneider, D. (2012). Ecological and evolutionary dynamics of coexisting lineages during a long-term experiment with 
Escherichia coli. Proceedings of the National Academy of Sciences of the United States of America, 109(24), 9487-9492.

Lenski, R. E., Rose, M. R., Simpson, S. C., \& Tadler, S. C. (1991). Long-Term Experimental Evolution in Escherichia coli . I . Adaptation and Divergence during 2,000 Generations. The American Naturalist, 138(6), 1315-1341.

Lenski, R. E., \& Travisano, M. (1994). Dynamics of adaptation and diversification: a 10,000-generation experiment with bacterial populations. Proceedings of the National Academy of Sciences of the United States of America, 91(15), 6808-6814.

Lewontin, R. C. (1978). Adaptation. Scientific American, 239(3), 212-231.

Li, H., \& Durbin, R. (2009). Fast and accurate short read alignment with Burrows-Wheeler transform. Bioinformatics, 25(14), 1754-1760.

Lind, P. A., Farr, A. D., \& Rainey, P. B. (2015). Experimental evolution reveals hidden diversityin evolutionary pathways. ELife, 2015(4), 1-17.

Lustenhouwer, N., Maynard, D. S., Bradford, M. A., Lindner, D. L., Oberle, B., Zanne, A. E., \& Crowther, T. W. (2020). A trait-based understanding of wood decomposition by fungi. Proceedings of the National Academy of Sciences of the United States of America, 117(21), 1-8.

Maas, M. F. P. M., Boer, H. J. D., Debets, A. J. M., \& Hoekstra, R. F. (2004). The mitochondrial plasmid pAL2-1 reduces calorie restriction mediated life span extension in the filamentous fungus Podospora anserina. Fungal Genetics and Biology, 41(9), 865-871.

Macheleidt, J., Mattern, D. J., Fischer, J., Netzker, T., Weber, J., Schroeckh, V., Valiante, V., \& Brakhage, A. A. (2016). Regulation and Role of Fungal Secondary Metabolites. Annual Review of Genetics, 50, 371-392.

MacLean, R. C. (2005). Adaptive radiation in microbial microcosms. Journal of Evolutionary Biology, 18(6), 1376-1386.

Maddamsetti, R., Lenski, R. E., \& Barrick, J. E. (2015). Adaptation, clonal interference, and frequency-dependent interactions in a long-term evolution experiment with escherichia coli. Genetics, 200(2), 619-631.

Martin, J. F., \& Gutierrez, S. (1992). Molecular genetics of fungal secondary metabolites. In Applied Molecular Genetics of Filamentous Fungi.

Martinelli, D. S., \& Clutterbuck, A. J. (1971). A quantative survey of conidiation mutants in Aspergillus nidulans. Journal of General Microbiology, 69, 261-268.

Maynard Smith, J., \& Szathmary, E. (1995). The Major Transitions In Evolution. Oxford University Press.

McKenna, A., Hanna, M., Banks, E., Sivachenko, A., Cibulskis, K., Kernytsky, A., Garimella, K., Altshuler, D., Gabriel, S., Daly, M., \& DePristo, M. A. (2010). The Genome Analysis Toolkit: A MapReduce framework for analyzing next-generation DNA sequencing data. Genome Research, 20, 1297--1303. 
Meunier, C., Hosseini, S., Heidari, N., Maryush, Z., \& Johannesson, H. (2018). Multilevel selection in the filamentous ascomycete Neurospora tetrasperma. American Naturalist, 191(3), 290-305.

Meyer, J. R., Dobias, D. T., Medina, S. J., Servilio, L., Gupta, A., \& Lenski, R. E. (2016). Ecological speciation of bacteriophage lambda in allopatry and sympatry. Science, 354(6317), 1301-1304.

Michod, R. E. (2005). On the transfer of fitness from the cell to the multicellular organism. Biology and Philosophy, 20, 967-987.

Mooney, J. L., \& Yager, L. N. (1990). Light is required for conidiation in Aspergillus nidulans. Genes and Development, 4(9), 1473-1482.

Morris, J. J., Papoulis, S. E., \& Lenski, R. E. (2014). Coexistence of evolving bacteria stabilized by a shared Black Queen function. Evolution, 68(10), 2960-2971.

Muller, H. (1932). Some genetic aspects of sex. The American Naturalist, 66(703), 118-138.

Nieuwenhuis, B. P. S., \& Aanen, D. K. (2018). Nuclear arms races: Experimental evolution for mating success in the mushroom-forming fungus Schizophyllum commune. PLOS ONE, 13(12).

Noble, L. M., \& Andrianopoulos, A. (2013). Reproductive competence: A recurrent logic module in eukaryotic development. Proceedings of the Royal Society B: Biological Sciences, 280(1766).

Noble, L. M., Holland, L. M., McLauchlan, A. J., \& Andrianopoulos, A. (2016). A plastic vegetative growth threshold governs reproductive capacity in Aspergillus nidulans. Genetics, 204(3), 1161-1175.

Novak, M., Pfeiffer, T., Lenski, R. E., Sauer, U., \& Bonhoeffer, S. (2006). Experimental Tests for an Evolutionary Trade-off between Growth Rate and Yield in E. coli. In The American Naturalist (Vol. 168, Issue 2, p. 242).

Novella, I. S., Duarte, E. A., Elena, S. F., Moya, A., Domingo, E., \& Holland, J. J. (1995). Exponential increases of RNA virus fitness during large population transmissions. Proceedings of the National Academy of Sciences of the United States of America, 92(13), 5841-5844.

Novick, A., \& Szilard, L. (1950). Experiments with the Chemostat on Spontaneous Mutations of Bacteria. Proceedings of the National Academy of Sciences, 36(12), 708-719.

Okasha, S. (2005). Multilevel selection and the major transitions in evolution. Philosophy of Science, 72(5), 1013-1025.

Paquin, C., \& Adams, J. (1983). Relative fitness can decrease in evolving populations of S. cerevisiae. Nature, 306(24), 368-371.

Park, H. S., \& Yu, J. H. (2012). Genetic control of asexual sporulation in filamentous fungi. Current Opinion in Microbiology, 15(6), 669-677.

Park, H. S., \& Yu, J. H. (2016). Velvet regulators in aspergillus spp. Microbiology and Biotechnology Letters, 44(4), 409-419. 
Paterson, S., Vogwill, T., Buckling, A., Benmayor, R., Spiers, A. J., Thomson, N. R., Quail, M., Smith, F., Walker, D., Libberton, B., Fenton, A., Hall, N., \& Brockhurst, M. A. (2010). Antagonistic coevolution accelerates molecular evolution. Nature, 464(7286), 275-278.

Perfeito, L., Pereira, M. I., Campos, P. R. A., \& Gordo, I. (2008). The effect of spatial structure on adaptation in Escherichia coli. Biology Letters, 4(1), 57-59.

Pfeiffer, T., Schuster, S., \& Bonhoeffer, S. (2001). Cooperation and Competition in the Evolution of ATP-Producing Pathways. Science, 292, 504-508.

Philippe, N., Crozat, E., Lenski, R. E., \& Schneider, D. (2007). Evolution of global regulatory networks during a long-term experiment with Escherichia coli. BioEssays, 29(9), 846860.

Pineda-Krch, M., \& Lehtilä, K. (2004). Costs and benefits of genetic heterogeneity within organisms. Journal of Evolutionary Biology, 17(6), 1167-1177.

Poltak, S. R., \& Cooper, V. S. (2011). Ecological succession in long-term experimentally evolved biofilms produces synergistic communities. ISME Journal, 5(3), 369-378.

Pontecorvo, G. (1953). The genetics of Aspergillus nidulans. In M. Demerec (Ed.), Advances in genetics (Vol. 5, pp. 141-238). Academic Press. Inc.

Pontecorvo, G. (1956). The parasexual cycle in fungi. Annual Review of Microbiology, 10, 393-400.

Pontecorvo, G., Roper, J. A., \& Forbes, E. (1953). Genetic recombination without sexual reproduction in Aspergillus niger. Journal of General Microbiology, 8(1), 198-210.

Pringle, A., \& Taylor, J. W. (2002). The fitness of filamentous fungi. Trends in Microbiology, 10(10), 474-481.

Rainey, P. B., Buckling, A., Kassen, R., \& Travisano, M. (2000). The emergence and maintenance of diversity: Insights from experimental bacterial populations. Trends in Ecology and Evolution, 15(6), 243-247.

Rainey, P. B., \& Travisano, M. (1998). Adaptive radiation in a heterogenous environment. Nature, 394, 69-72.

Read, N. D., Fleißner, A., Roca, G. M., \& Glass, L. N. (2010). Hyphal fusion. In J. Ebbole, Daniel \& K. A. Borkovich (Eds.), Cellular and Molecular Biology of Filamentous Fungi (pp. 260273). ASM Press.

Rech, C., Engh, I., \& Kück, U. (2007). Detection of hyphal fusion in filamentous fungi using differently fluorescence-labeled histones. Current Genetics, 52(5-6), 259-266.

Rendueles, O., Amherd, M., \& Velicer, G. J. (2015). Positively Frequency-Dependent Interference Competition Maintains Diversity and Pervades a Natural Population of Cooperative Microbes. Current Biology, 25(13), 1673-1681.

Reznick, D. (1983). The Structure of Guppy Life Histories: The Tradeoff between Growth and Reproduction. Ecology, 64(4), 862-873.

Riquelme, M., Aguirre, J., Bartnicki-García, S., Braus, G. H., Feldbrügge, M., Fleig, U., Hansberg, W., Herrera-Estrella, A., Kämper, J., Kück, U., Mouriño-Pérez, R. R., Takeshita, N., \& Fischer, R. (2018). Fungal Morphogenesis, from the Polarized Growth of Hyphae 
to Complex Reproduction and Infection Structures. Microbiology and Molecular Biology Reviews, 82(2), 1-47.

Ritz, K. (1995). Growth responses of some soil fungi to spatially heterogeneous nutrients. FEMS Microbiology Ecology, 16, 269-280.

Robinson, J. T., Thorvaldsdóttir, H., Winckler, W., Guttman, M., Lander, E. S., Getz, G., \& Mesirov, J. P. (2011). Integrative genomics viewer. Nature Biotechnology, 29(1), 24-26.

Roff, D. A. (2000). Trade-offs between growth and reproduction: An analysis of the quantitative genetic evidence. In Journal of Evolutionary Biology (Vol. 13, Issue 3, pp. 434-445).

Rogers S.O., B. A. J. (1994). Plant Molecular biology manual. In Springer, Dordrecht.

Rosenzweig, F., Sharp, R. R., Treves, D. S., \& Adams, J. (1994). Microbial evolution in a simple unstructured environment: Genetic differentiation in Escherichia coli. Genetics, 137(4), 903-917.

Rozen, D. E., \& Lenski, R. E. (2000). Long-term experimental evolution in Escherichia coli. VIII. Dynamics of a balanced polymorphism. In American Naturalist (Vol. 155, Issue 1, pp. 24-35).

Rozen, D. E., Philippe, N., De Visser, J. A. G. M., Lenski, R. E., \& Schneider, D. (2009). Death and cannibalism in a seasonal environment facilitate bacterial coexistence. Ecology Letters, 12(1), 34-44.

Rozen, D. E., Schneider, D., \& Lenski, R. E. (2005). Long-term experimental evolution in Escherichia coli. XIII. Phylogenetic history of a balanced polymorphism. Journal of Molecular Evolution, 61(2), 171-180.

Santelices, B. (1999). How many kinds of individual are there? Trends in Ecology and Evolution, 14(4), 152-155.

Sathe, S., \& Nanjundiah, V. (2018). Complex interactions underpin social behaviour in Dictyostelium giganteum. Behavioral Ecology and Sociobiology, 72(10).

Saxer, G., Doebeli, M., \& Travisano, M. (2009). Spatial structure leads to ecological breakdown and loss of diversity. Proceedings of the Royal Society B: Biological Sciences, 276(1664), 2065-2070.

Saxer, G., Doebeli, M., \& Travisano, M. (2010). The repeatability of adaptive radiation during long-term experimental evolution of Escherichia coli in a multiple nutrient environment. PLOS ONE, 5(12).

Schluter, D., Price, T. D., \& Rowe, L. (1991). Conflicting selection pressures and life history trade-offs. Proceedings of the Royal Society B: Biological Sciences, 246(1315), 11-17.

Schmit, J. P. (2002). Tradeoffs between reproduction and mycelium production in the unitrestricted decomposer Coprinus cinereus. Mycologia, 94(1), 40-48.

Schoustra, S. E., Bataillon, T., Gifford, D. R., \& Kassen, R. (2009). The properties of adaptive walks in evolving populations of fungus. PLoS Biology, 7(11). 
Schoustra, S. E., Debets, A. J. M., Slakhorst, M., \& Hoekstra, R. F. (2006). Reducing the cost of resistance; experimental evolution in the filamentous fungus Aspergillus nidulans. Journal of Evolutionary Biology, 19(4), 1115-1127.

Schoustra, S. E., Debets, A. J. M., Slakhorst, M., \& Hoekstra, R. F. (2007). Mitotic recombination accelerates adaptation in the fungus Aspergillus nidulans. PLoS Genetics, 3(4), 06480653.

Schoustra, S. E., Hwang, S., Krug, J., \& De Visser, J. A. G. M. (2016). Diminishing-returns epistasis among random beneficial mutations in a multicellular fungus. Proceedings of the Royal Society B: Biological Sciences, 283(1837).

Schoustra, S. E., \& Punzalan, D. (2012). Correlation of mycelial growth rate with other phenotypic characters in evolved genotypes of Aspergillus nidulans. Fungal Biology, 116(5), 630-636.

Schoustra, S. E., Slakhorst, M., Debets, A. J. M., \& Hoekstra, R. F. (2005). Comparing artificial and natural selection in rate of adaptation to genetic stress in Aspergillus nidulans. Journal of Evolutionary Biology, 18(4), 771-778.

Smith, N. W., Shorten, P. R., Altermann, E., Roy, N. C., \& McNabb, W. C. (2019). The Classification and Evolution of Bacterial Cross-Feeding. Frontiers in Ecology and Evolution, 7(May), $1-15$.

Stearns, S. C. (1989). Trade-Offs in Life-History Evolution. Function Ecology, 3(3), 259-268.

Stearns, S. C. (2000). Life history evolution: Successes, limitations, and prospects. Naturwissenschaften, 87(11), 476-486.

Strassmann, J. E., Zhu, Y., \& Queller, D. C. (2000). Altruism and social cheating in the social amoeba Dictyostelium discoideum. Nature, 408(6815), 965-967.

Swift, R. J., Craig, S. H., Wiebe, M. G., Robson, G. D., \& Trinci, A. P. J. (2000). Evolution of Aspergillus niger and A. nidulans in glucose-limited chemostat cultures, as indicated by oscillations in the frequency of cycloheximide resistant and morphological mutants. Mycological Research, 104(3), 333-337.

Tarnita, C. E., Washburne, A., Martinez-Garcia, R., Sgro, A. E., \& Levin, S. A. (2015). Fitness tradeoffs between spores and nonaggregating cells can explain the coexistence of diverse genotypes in cellular slime molds. Proceedings of the National Academy of Sciences, 112(9), 2776-2781.

Tenaillon, O., Barrick, J. E., Ribeck, N., Deatherage, D. E., Blanchard, J. L., Dasgupta, A., Wu, G. C., Wielgoss, S., Cruveiller, S., Médigue, C., Schneider, D., \& Lenski, R. E. (2016). Tempo and mode of genome evolution in a 50,000-generation experiment. Nature, 536(7615), 165-170.

Traverse, C. C., Mayo-Smith, L. M., Poltak, S. R., \& Cooper, V. S. (2013). Tangled bank of experimentally evolved Burkholderia biofilms reflects selection during chronic infections. Proceedings of the National Academy of Sciences of the United States of America, 110(3). 
Treves, D. S., Manning, S., \& Adams, J. (1998). Repeated evolution of an acetate-crossfeeding polymorphism in long-term populations of Escherichia coli. Molecular Biology and Evolution, 15(7), 789-797.

Trienens, M., \& Rohlfs, M. (2011). Experimental evolution of defense against a competitive mold confers reduced sensitivity to fungal toxins but no increased resistance in Drosophila larvae. BMC Evolutionary Biology, 11(1).

Tuomi, J., \& Vuorisalo, T. (1989a). Hierarchical selection in modular organisms. Trends in Ecology and Evolution, 4(7), 209-213.

Tuomi, J., \& Vuorisalo, T. (1989b). What Are the Units of Selection in Modular Organisms? Oikos, 54(2), 227-233.

Turner, C. B., Blount, Z. D., Mitchell, D. H., \& Lenski, R. E. (2015). Evolution and coexistence in response to a key innovation in a long-term evolution experiment with Escherichia coli. BioRxiv, 020958.

Turner, P. E., Souza, V., \& Lenski, R. E. (1996). Tests of Ecological Mechanisms Promoting the Stable Coexistence of Two Bacterial Genotypes. Ecology, 77(7), 2119-2129.

Van De Vondervoort, P. J. I., Poulsen, B. R., Ruijter, G. J. G., Schuleit, T., Visser, J., \& Iversen, J. J. L. (2004). Isolation of a Fluffy Mutant of Aspergillus niger from Chemostat Culture and Its Potential Use as a Morphologically Stable Host for Protein Production. Biotechnology and Bioengineering, 86(3), 301-307.

Van den Bergh, B., Swings, T., Fauvart, M., \& Michiels, J. (2018). Experimental Design , Population Dynamics, and Diversity in microbial experimental evolution. Microbiology and Molecular Biology Reviews, 82(3), 1-54.

Van Dijk, B., Meijer, J., Cuypers, T. D., \& Hogeweg, P. (2019). Trusting the hand that feeds: Microbes evolve to anticipate a serial transfer protocol as individuals or collectives. BMC Evolutionary Biology, 19(1), 1-18.

van Noordwijk, A. ., \& de Jong, G. (1986). Acquisition and Allocation of Resources: Their Influence on Variation in Life History Tactics. The American Naturalist, 128(1), 137-142.

Vasi, F., Travisano, M., \& Lenski, R. E. (1994). Long-Term Experimental Evolution in Escherichia coli . II . Changes in Life-History Traits During Adaptation to a Seasonal Environment Author ( $\mathrm{s}$ ): Farida Vasi , Michael Travisano and Richard E . Lenski Published by: The University of Chicago Press for. The American Naturalist, 144(3), 432-456.

Velicer, G. J., Kroos, L., \& Lenski, R. E. (2000). Developmental cheating in the social bacterium Myxococcus xanthus. Nature, 404(6778), 598-601.

Velicer, G. J., \& Lenski, R. E. (2007). Evolutionary Trade-Offs under Conditions of Resource Abundance and Scarcity: Experiments with Bacteria EVOLUTIONARY TRADE-OFFS UNDER CONDITIONS OF RESOURCE ABUNDANCE AND SCARCITY : EXPERIMENTS WITH BACTERIA. 80(4), 1168-1179.

Vuorisalo, T., \& Tuomi, J. (1986). Unitary and Modular Organisms : Criteria for Ecological Division. Oikos, 47(3), 382-385. 
Whiteway, M., \& Bachewich, C. (2005). Fungi:Biology and applications. In K. Kavanagh (Ed.), Fungal genetics (pp. 35-64). John Wiley and Sons,Ltd.

Wiser, M. J., Ribeck, N., \& Lenski, R. E. (2013). Long-term dynamics of adaptation in asexual populations. Science, 342(December), 1364-1367.

Withers, J. M., Wiebe, M. G., Robson, G. D., \& Trinci, A. P. J. (1994). Development of morphological heterogeneity in glucose-limited chemostat cultures of Aspergillus oryzae. Mycological Research, 98(1), 95-100.

Wloch-Salamon, D. M., Gerla, D., Hoekstra, R. F., \& De Visser, J. A. G. M. (2008). Effect of dispersal and nutrient availability on the competitive ability of toxin-producing yeast. Proceedings of the Royal Society B: Biological Sciences, 275(1634), 535-541.

Wolf, J. B., Howie, J. A., Parkinson, K., Gruenheit, N., Melo, D., Rozen, D. E., \& Thompson, C. R. L. (2015). Fitness trade-offs result in the illusion of social success. Current Biology, 25(8), 1086-1090.

$\mathrm{Yu}$, J.-H. (2010). Regulation of Development in Aspergillus nidulans and Aspergillus fumigatus . Mycobiology, 38(4), 229.

Yu, L., Boström, C., Franzenburg, S., Bayer, T., Dagan, T., \& Reusch, T. B. H. (2020). Somatic genetic drift and multilevel selection in a clonal seagrass. Nature Ecology and Evolution, 4(7), 952-962.

Zera, A. J., \& Harshman, L. G. (2001). The Physiology of Life History Trade-Offs in Animals. Annual Review of Ecology and Systematics, 32(2001), 95-126.

Zhang, J., Debets, A. J. M., Verweij, P. E., Melchers, W. J. G., Zwaan, B. J., \& Schoustra, S. E. (2015). Asexual sporulation facilitates adaptation: The emergence of azole resistance in Aspergillus fumigatus. Evolution, 69(10), 2573-2586.

Zhang, J., Van Den Heuvel, J., Debets, A. J. M., Verweij, P. E., Melchers, W. J. G., Zwaan, B. J., \& Schoustra, S. E. (2017). Evolution of cross-resistance to medical triazoles in Aspergillus fumigatus through selection pressure of environmental fungicides. Proceedings of the Royal Society B: Biological Sciences, 284(1863).

Zheng, W., Lehmann, A., Ryo, M., Vályi, K. K., \& Rillig, M. C. (2020). Growth rate trades off with enzymatic investment in soil filamentous fungi. Scientific Reports, 10(1), 8-13.

Zwaan, B. J., Bijlsma, R., \& Hoekstra, R. F. (1995). Direct selection on life span in Drosophila melanogaster. Evolution, 49(4), 649-659. 

Summary 
Life-history theory tries to explain and understand how evolution shapes an organism's reproductive success, driven in part by trade-offs among life-history traits. A central concept that has been used to explain trade-offs is the differential allocation of limited resources to different fitness-related traits, such as the timing and extent of reproduction and longevity.

What is an optimal life-history strategy for a filamentous fungus? Theory predicts that the life-history strategy that a filamentous fungus employs depends on the quality and quantity of available resources and external cause of mortality for example, the presence of competitors. A fungal colony growing on rich, short-lived resources, such as dung or decaying fruit, may first only invest in somatic growth and then switch to reproduction (iteroparous reproduction). Alternatively, when growing on long-lived resources that are harder to break down, like dead tree trunks, growth may be slower and continues after the onset of reproduction, and reproduction is generally a recurring event (semelparous reproduction).

In this thesis, I used the filamentous ascomycete fungus Aspergillus nidulans to test the role of trade-offs between somatic growth and asexual reproduction during adaptation to a new environment. The life cycle of $A$. nidulans starts with the germination of a haploid spore, which grows into a multicellular mycelial network that upon internal and external stimuli produces asexual, and later also sexual, spores.

The $A$. nidulans strains that I used have two different origins. In one set of experiments, I used a collection of natural isolates to test for a trade-off between growth and asexual reproduction during adaptation to their natural environment. I then used a subset of the isolates to test for trade-offs between growth and asexual reproduction during short-term laboratory evolution. In the second set of experiments, I used a laboratory strain of $A$. nidulans in a one-year evolution experiment to examine adaptive constrains more generally, including the role of trade-offs between growth and asexual reproduction.

In chapter 2, I tested the role of trade-offs between growth rate and asexual reproduction in two ways. First, I measured the mycelium growth rate (MGR) and asexual spore yield of fifteen natural isolates of $A$. nidulans in four laboratory environments. A trade-off here would reflect adaptive constraints during evolution in their natural environment. However, no significant correlation between MGR and asexual spore yield was observed. Instead, there was a clear dependence of the two traits on sugar concentration and nutrient richness. I then selected six isolates for a short evolution experiment in one environment where 
the natural isolates showed the most variation in MGR and spore yield. Among the independently evolved replicate populations, a negative correlation between growth rate and spore density was observed that approached significance, but not between growth rate and spore yield. Next, I tested whether changes in both traits were dependent on their initial value. I saw five strains with low MGR improved and one strain with highest MGR declined. All changes occurred in an antagonistic fashion: increases in one (mostly MGR) were associated with decreases in spore yield/density, and vice versa.

In chapters 3 to 5,1 examined the scope and mechanisms of adaptation of $A$. nidulans in a simple laboratory environment, including the role of trade-offs between growth rate and asexual reproduction. For this, 12 populations were initiated from a single strain and propagated for one year via weekly transfer of asexual spores to fresh minimal glucose medium, dubbed Aspergillus Short-term Evolution eXperiment (ASEX).

Although $1 \%$ of asexual spores were transferred every week for a year, in chapter 3 , I surprisingly found a consistent statistically significant reduction in asexual spore yield in all populations over time. When I tested the role of trade-offs between growth rate and reproduction over evolutionary time, I found no correlation between changes in MGR and spore yield. Even more surprisingly, the competitive fitness of the evolved populations relative to their ancestor had not consistently improved compared either. However, fitness measurements for two populations using more recent predecessors as competitors suggested that non-transitive fitness interactions explained the lack of fitness increases relative to the ancestor. Upon closer inspection, all populations had evolved morphological polymorphism as early as week 20 . The two morphotypes, named ancestor-like (AL) and fluffy-like (FL), did not show consistent differences in MGR and spore yield.

In chapter 4, I explored possible mechanisms responsible for the maintenance of the two morphotypes. I did competition experiments between the two types in head-to-head competitions at low and high frequencies. The competition results showed that the two types were engaged in negative frequency-dependent fitness interactions, but these could not fully explain their observed frequencies in the evolving populations. I next tested whether positive (cross-feeding) or negative (interference competition) metabolic interactions between the two types were involved, using a conditioned medium, which showed that FL is a superior competitor for the limiting nutrient (glucose). 
In chapter 5, I explored the genomic basis of adaptation of the ASEX populations, based on sequence analysis of clones from both morphotypes of all 12 populations at week 10 and 52 of the experiment. The overall ratio of $d N / d S$ across the genome of all clones was not significantly different from a neutral expectation. However, I did find that genes that were mutated in two or more populations, occurred more frequently than expected by chance, indicating that they were under positive selection. Among the seven ASEX populations where both morphs persisted until the end of the experiment, in only one population both morphs were monophyletic, while in the other six populations the two morphs from the final time point derived from unrelated genetic backgrounds, suggesting their frequent extinction and re-emergence. Of the eight parallelly mutated genes in our populations, veA and orthologs of AN2701 and AN5776 are well characterized. veA is a fungal transcription factor involved in the light-dependent regulation of sexual and asexual reproduction and secondary metabolism. The orthologs of two other parallel muted genes, $A N 2701$ and $A N 5776$, are the well-characterized $N$. crassa ham-5 and soft genes. The fusion tests of our populations showed that mutations in $A$. nidulans AN2701 and AN5776 also reduce hyphal fusion. Fusion between vegetative hyphae is known to be essential for intra-hyphal communication and during sexual and para sexual reproduction.

In conclusion, I found little evidence for a trade-off between growth and reproduction in $A$. nidulans. In both laboratory evolution studies, I found a reduction in asexual spore yield and variable changes in mycelial growth rate, questioning their general role during in adaptation in this filamentous fungus. Evolution in a simple structured environment can rapidly generate and sustain heterogeneity within populations of $A$. nidulans via competition for resources. The deletion of fusion genes in the final time-point clones might play a role in hyphal communication and sexual reproduction along with mutations in veA.

Fungi occupy a wide range of niches and are essential players in recycling carbon and nitrogen in the biosphere. It is crucial to understand which components of fitness contribute to adaptation to these different ecological conditions. For this, we need to collect data from diverse fungal isolates from different niches and not restrict ourselves to studying a few well-established fungal model systems and develop fungal-specific life-history models. 

Acknowledgements 
At last, the thesis is done. Over the years I have met a lot of wonderful people who have made this journey a memorable one. I am not good at expressing my feelings, so I'm afraid the acknowledgement might sound like a generic one. However, there is nothing generic about the bond that I share with each and everyone of you. It's the moments that I've shared with all of you that has shaped my personality and has added a lot of colour and brightness to my life. So here's an attempt at saying a big thank you to everyone for their friendship, support and encouragement.

I would like to dedicate this thesis to my mom and my late uncle Jagadeesh. I would not have been on this journey if it wasn't for their support and faith in me. This journey has been a roller-coaster ride for my mom and me; thank you ma for joining me on this journey.

Many thanks are due to my promotors and co-promotor. I am lucky to have found an amazing group of advisors who are amazing scientists, easy to talk to and who do not micromanage. Thank you for your support and for giving me a lot of space and freedom to shape the project. Sure, it took me a while to get here, but I would not have preferred it any other way. Special thanks to Arjan and Duur for the many hours of online meetings. I've really appreciated your patience when I was slow in understanding your expectations and for accepting my inability to plan things ahead.

Sijmen and Fons, thank you for your interest and enthusiasm in my project, for the many discussions we have had for troubleshooting, for analyzing and organizing the chapters and also for being such fun and easy-going people to hang out with.

Special thanks to the other members of my thesis committee Jan Kammenga, Marjon deVos, Han Wösten and Anna van Diepeningen for reviewing this thesis.

My journey in academic research started when Mahi sir, gave me an opportunity to work in his lab and he has become my unofficial advisor. Thank you for welcoming me into your lab, for giving me labspace to work on my PhD project and for giving me the same attention and time as your other students. I would like to thank all the past members of Mahi and VN labs at MRDG and the BEE lab at MCB, IISC.

I have been working with VN long before the PhD project started, I have learned so much from you, thank you. You have treated me like a family member and I have enjoyed the annual cultural evening you host, the postcards you've sent from all the places you visited and the yummy goodies that you brought for all the festivals. 
A special mention goes to Stuti and Ritwick for training me in bacterial and, Dictyostelium genetics, and for the amazing time we have shared; to Parisa for being my confidant and for your constant support and encouragement. I hope someday we will be in the same country/city once again. My soul-twin Prathibha, thank you for everything. Tejas, thanks for always giving me an outsider's perspective when things got frustrating. Kru, aunty and uncle, thank you for the relaxing evenings I've spent at your home. My MCC gals Su, Shantu, Laas and Geets, we've come a long way and we have many more seasons in life to share.

The decision to move (even for a short while) to a different continent was a big one because I had lived in Bangalore my entire life. Looking back, I am so glad that I made this brave decision because it has been the best and the most transformative journey of my life.

Bas, thank you for being a real-life Dumbledore and attracting such a wonderful group of people at the Laboratory of Genetics and for always being involved in all our projects and well-being. The atmosphere in the Laboratory of Genetics is nurturing, friendly, relaxed and supportive; it's a great place to do science and make friends. I have had a wonderful time discussing science and every topic under the sun with all the past and present members of the group. The discussions we've had during coffee and lunch breaks were the best.

Wytske, thank you for being you and keeping us all together and for your patience when I submitted bills, documents and paperwork at the last minute.

I have to thank Marijke for her no-nonsense way of managing the lab and for teaching me the ins and outs of fungal work, using the big scary autoclave on the first floor and for showing me where the glassware washing room is so that I could, on occasion hijack the glassware before they reached our cupboards.

Over the years, I have depended on all the lab managers Bertha, Gabriella, Frank, Jose, Corrie, Lidia and Jordy for both their expertise and friendly company. Thank you for your support.

Eric, I am glad that you are managing the fungal lab now. Thank you for your calming presence and for helping me out with all my experiments, from brainstorming the design to helping with the preparation and execution of the experiments. 
Joost and Ben, thank you for analyzing the bioinformatics data. Julia, thanks for choosing to do your bachelor's project with us and working on the natural isolates, I enjoyed working with you.

Ben, you have taken the role of being my unofficial advisor and I have leaned on you so much these past few years. Thank you for your interest in my project and for making the time for the many discussions I have had about the data and how to present them (esp. the spent medium data), for the nice figures and for showing me to how to take good quality images that are on the cover page.

I have awesome memories of not just doing good science, but also the amazing social circle I have found at the Laboratory of Genetics. Thank you, Eric, Jianhua, Claudio, Lidia, Alex, Anna, Ben, Jenny, Gabriella, Suzette, Eveline, Kim, Margo, Anneloes, Manja, Sarah and Sabine for the many amazing picnics, game-nights, movie-nights, visits to museums, zoos and escape rooms and exploring The Netherlands with me.

Anneloesje, thank you, for the many fries and kroketten dinners at Het Ambacht and for the crazy intense workout sessions we signed up for at the sports center deBongerd (-).

Suzette and Manja, thank you keeping my sweet tooth and me happy with the vegan/eggless brownies, tarts, shortbread and all the other yummy baked goodies.

Kim and Margo thank you for introducing me to escape rooms. Sarah, thank you for exploring Wageningen and its surroundings with me.

Jianhua, thank you for the swimming lessons we took together, I am not a good swimmer yet, but at least I won't sink like a rock if I accidentally fall into the water.

Gabriella, Eveline, Manja and Kim I have had so much fun discussing books, life and everything else with all of you.

Alex and Anna, thank you for taking me on the best road trip to Montpellier. It was one exciting trip.

I can't thank you all enough for checking-up on me and taking the time to meet up for walks (with social distancing) during the first wave of the pandemic when I was stuck in The Netherlands for nearly six months. 
I have been lucky to meet amazing people outside the Laboratory of Genetics too. A special shout-out to the Wageningen Ultimate frisbee group- team DiscOver! Martijn, Tanja, Bram, Anneke, Thierry, Elyssa and Rick. I have enjoyed being a part of the team and driving all over The Netherlands to play at tournaments, the postgame pizzas and the practice sessions. Another shout-out to the IISc Ultimate frisbee group- team SlipdIISc. Ultimate frisbee like Laboratory of Genetics attracts the friendliest people in the world.

The Indian I've met in Wageningen Anoop and gang, thank you for introducing me to yummy North Indian home food and helping me improve my Hindi. Manjunath and Kavitha thank you for letting me crash at your home in Zetten and feeding me traditional home-cooked meals and for the chance to speak in Kannada. Spending time with you made me feel like I was in Bangalore and not in The Netherlands.

A big thank you to all my friends and family for driving me around Bangalore when I've been lazy (plenty of times) to use my bromfiets or the public transport.

I can't end this acknowledgment without thanking my friends F2 generation: Jack, Lisa, Kris, Vincent, Sebastian, Dhruva, Aarush, Thomas, Rachit, Sarvajeet and Samrudh; thank you for teaching me the names of Dutch farm animals, sharing your toys and games and introducing me to new cartoons and nursery rhymes. Spending time with all of you has helped make bad days better. 


\section{PE\&RC Training and Education Statement}

With the training and education activities listed below the $\mathrm{PhD}$ candidate has complied with the requirements set by the C.T. de Wit Graduate School for Production Ecology and Resource Conservation (PE\&RC) which comprises of a minimum total of 32 ECTS (= 22 weeks of activities)

\section{Review of literature (4.5 ECTS)}

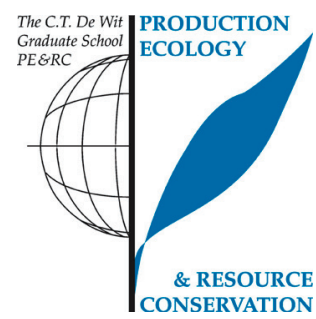

Cellular slime mould and a filamentous fungus as a model to study evolution of multicellularity

\section{Writing of Project proposal (4.5 ECTS)}

Causes and constraints in the early evolution of multicellularity

\section{Post-graduate courses (4.5 ECTS)}

Experimental evolution, theory and practices; ENS, Paris (2016)

- School on population genetics and evolution; ICTS, India (2016)

\section{Laboratory training and working visits (4.5 ECTS)}

Using Myxococcus xanthus as a model system to study trade-offs in life history traits; Indian Institute of Science (2019-2020)

\section{Deficiency, refresh, brush-up courses (4.5 ECTS)}

Seminal papers in ecology and evolution; Laboratory of Genetics (2015)

Molecular and evolutionary ecology; Laboratory of Genetics (2015)

Basic statistics; Biometris, Mathematics and statistical methods (2017)

\section{Competence strengthening / skills courses (0.4 ECTS)}

\section{PhD Carousel; WGS (2017)}

- Reviewing a scientific paper; WGS (2017)

PE\&RC Annual meetings, seminars and the PE\&RC weekend (0.9 ECTS)

PE\&RC Final year weekend (2017)

PE\&RC Day (2018)

\section{Discussion groups / local seminars or scientific meetings (6.2 ECTS)}

Journal clubs at MRDG/CHG/MCB; India (2015, 2016, 2019)

WEES seminar; GEN $(2014,2017,2018)$

\section{International symposia, workshops and conferences (9.2 ECTS)}

Conflict and cooperation in cellular systems, CCCP (2016)

Netherlands Society for Evolutionary Biology, NLSEB (2018)

European society for evolutionary biology, ESEB (2018)

Westerdijk Fungal Biodiversity Institute annual fungal meeting (2018)

Conflict and cooperation in cellular systems, CCCP (2020)

\section{MSc thesis supervision (1.5 ECTS)}

Natural variation of mutation rate, sporulation and growth in filamentous fungi 
The research described in this thesis was carried out at the Laboratory of Genetics at Wageningen University, Wageningen, The Netherlands, and was financially supported by funding from Wageningen University and Research sandwich PhD programme and Centre for Human Genetics, Bangalore.

Financial support from Wageningen University for printing this thesis is gratefully acknowledged.

Cover design Krithi Nandimath

Thesis layout Dennis Hendriks || ProefschriftMaken.nl

Printed by

ProefschriftMaken.nl 


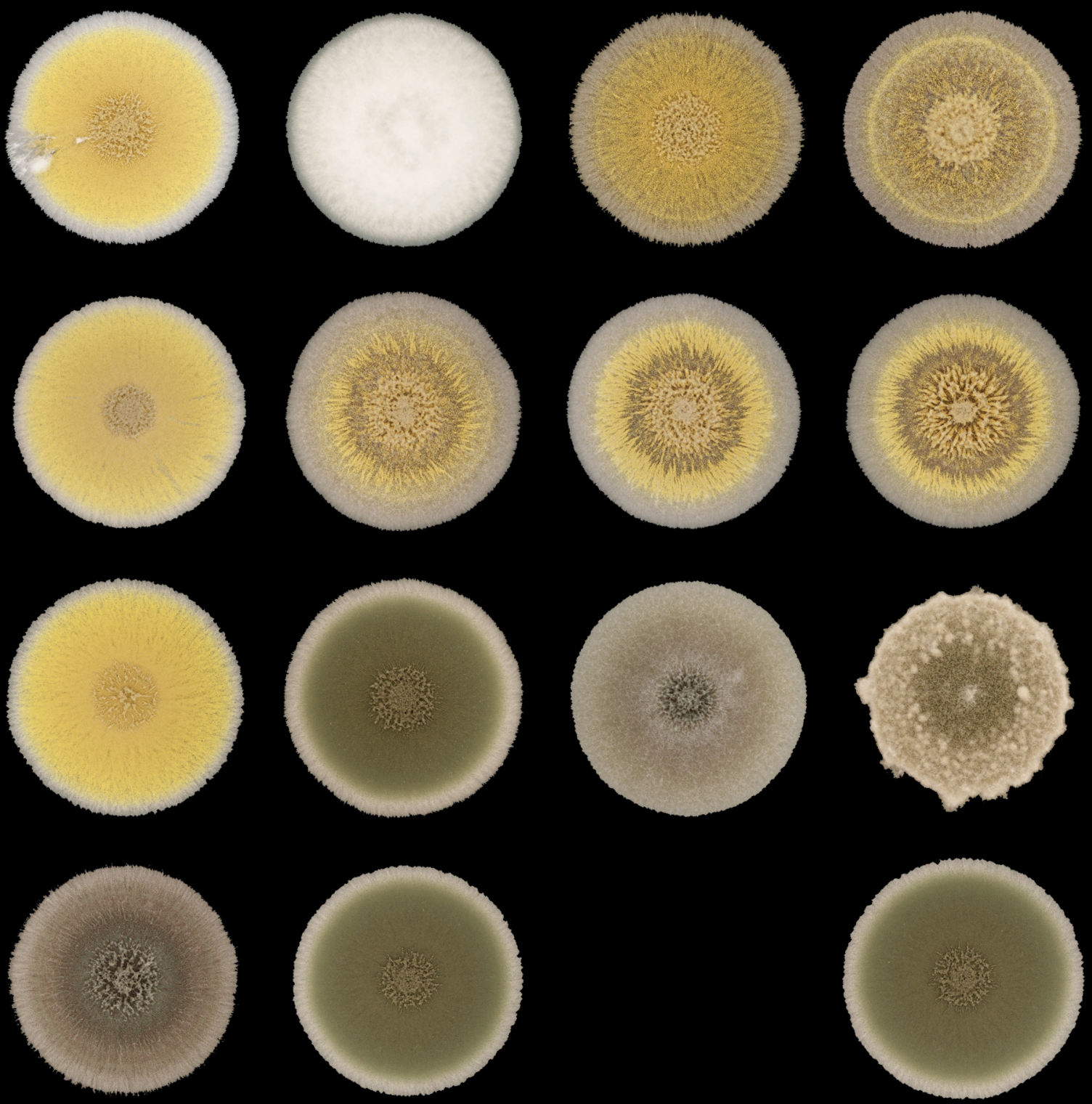